\title{
Organophosphine Oxide/Sulfide-Substituted Lanthanide Binaphtholate Catalysts for Enantioselective Hydroamination/Cyclization
}

\author{
Xianghua Yu and Tobin J. Marks* \\ Department of Chemistry, Northwestern University, 2145 Sheridan Road, Evanston, IL 60208 \\ Email: t-marks@northwestern.edu
}


Table S1. Crystal data and structure refinement for $\mathbf{1}$.

Identification code

Empirical formula

Formula weight

Temperature

Wavelength

Crystal system

Space group

Unit cell dimensions

Volume

Z

Density (calculated)

Absorption coefficient

$\mathrm{F}(000)$

Crystal size

Theta range for data collection

Index ranges

Reflections collected

Independent reflections

Completeness to theta $=28.48^{\circ}$

Absorption correction

Max. and min. transmission

Refinement method

Data / restraints / parameters

Goodness-of-fit on $\mathrm{F}^{2}$

Final R indices [I $>2 \operatorname{sigma(I)]~}$

$\mathrm{R}$ indices (all data)

Absolute structure parameter

Largest diff. peak and hole
1 (binol_1, BINOL-POPh 2. Pentane)

C51 H48 O4 P2

786.83

153(2) K

$0.71073 \AA$

Monoclinic

C2

$\mathrm{a}=16.114(3) \AA \quad \alpha=90^{\circ}$

$\mathrm{b}=18.939(3) \AA \quad \beta=98.317(4)^{\circ}$

$\mathrm{c}=25.987(5) \AA \quad \gamma=90^{\circ}$

$7847(2) \AA^{3}$

8

$1.332 \mathrm{~g} / \mathrm{cm}^{3}$

$0.160 \mathrm{~mm}^{-1}$

3328

$0.10 \times 0.06 \times 0.06 \mathrm{~mm}^{3}$

0.79 to $28.48^{\circ}$

$-21 \leq \mathrm{h} \leq 21,-24 \leq \mathrm{k} \leq 21,-31 \leq \mathrm{l} \leq 33$

23626

$15603[\mathrm{R}(\mathrm{int})=0.0661]$

$88.9 \%$

Semi-empirical from equivalents

0.9905 and 0.9842

Full-matrix least-squares on $\mathrm{F}^{2}$

15603 / 1 / 979

1.001

$\mathrm{R} 1=0.0700, \mathrm{wR} 2=0.1260$

$\mathrm{R} 1=0.1719, \mathrm{wR} 2=0.1489$

$0.10(10)$

0.644 and -0.331 e. $\AA^{-3}$ 
Table S2. Atomic coordinates $\left(\times 10^{4}\right)$ and equivalent isotropic displacement parameters $\left(\AA^{2} \times\right.$ $10^{3}$ ) for $\mathbf{1}$. $U(e q)$ is defined as one third of the trace of the orthogonalized $U^{i j}$ tensor.

\begin{tabular}{|c|c|c|c|c|}
\hline & $\mathrm{X}$ & $\mathrm{y}$ & $\mathrm{z}$ & $\mathrm{U}(\mathrm{eq})$ \\
\hline $\mathrm{C}(1)$ & $4163(4)$ & 4854(3) & $2367(2)$ & $36(1)$ \\
\hline $\mathrm{C}(2)$ & 4578(3) & 5321(3) & $2070(2)$ & $35(1)$ \\
\hline $\mathrm{C}(3)$ & $4263(4)$ & 5452(3) & $1538(2)$ & $36(1)$ \\
\hline $\mathrm{C}(4)$ & $3523(4)$ & 5125(3) & 1317(2) & $39(2)$ \\
\hline $\mathrm{C}(5)$ & $3094(4)$ & 4666(3) & $1605(2)$ & $42(2)$ \\
\hline $\mathrm{C}(6)$ & $2326(4)$ & 4332(3) & 1382(2) & $54(2)$ \\
\hline $\mathrm{C}(7)$ & 1941(5) & $3867(4)$ & 1670(3) & $75(2)$ \\
\hline $\mathrm{C}(8)$ & 2268(5) & $3716(4)$ & 2193(3) & $76(2)$ \\
\hline $\mathrm{C}(9)$ & 2993(4) & 4041(4) & 2419(2) & $58(2)$ \\
\hline$C(10)$ & $3427(4)$ & 4518(3) & 2133(2) & $38(2)$ \\
\hline$C(11)$ & $4541(3)$ & 4732(3) & $2927(2)$ & $35(1)$ \\
\hline $\mathrm{C}(12)$ & 5244(3) & 4329(3) & $3044(2)$ & $33(1)$ \\
\hline$C(13)$ & 5608(3) & 4218(3) & $3565(2)$ & $32(1)$ \\
\hline$C(14)$ & $5245(3)$ & 4497(3) & $3959(2)$ & $29(1)$ \\
\hline$C(15)$ & 4521(3) & 4912(3) & $3868(2)$ & $29(1)$ \\
\hline$C(16)$ & 4136(3) & 5202(3) & $4266(2)$ & $36(1)$ \\
\hline $\mathrm{C}(17)$ & $3420(4)$ & 5605(3) & 4171(2) & $42(2)$ \\
\hline C(18) & $3060(4)$ & 5704(3) & $3648(2)$ & $45(2)$ \\
\hline C(19) & $3406(4)$ & 5424(3) & $3253(2)$ & $44(2)$ \\
\hline$C(20)$ & $4140(3)$ & 5024(3) & $3339(2)$ & $33(1)$ \\
\hline $\mathrm{C}(21)$ & $4761(4)$ & 5890(3) & 1212(2) & $49(2)$ \\
\hline $\mathrm{C}(22)$ & $3758(4)$ & 7173(3) & 1191(2) & $51(2)$ \\
\hline$C(23)$ & $3261(4)$ & 6983(4) & 736(3) & $68(2)$ \\
\hline $\mathrm{C}(24)$ & $2460(5)$ & $7258(4)$ & 601(3) & $74(2)$ \\
\hline$C(25)$ & 2191(5) & $7760(4)$ & $921(4)$ & $79(2)$ \\
\hline$C(26)$ & 2662(6) & 7955(4) & 1372(3) & $85(3)$ \\
\hline $\mathrm{C}(27)$ & $3450(5)$ & $7662(4)$ & 1508(3) & $79(2)$ \\
\hline$C(28)$ & $5459(4)$ & 7238(4) & $962(2)$ & $56(2)$ \\
\hline C(29) & $6252(4)$ & $6936(4)$ & $925(3)$ & $78(2)$ \\
\hline C(30) & 6819(5) & 7271(5) & $658(3)$ & 89(3) \\
\hline $\mathrm{C}(31)$ & $6640(5)$ & 7923(5) & 446(3) & $72(2)$ \\
\hline C(32) & $5866(5)$ & $8228(4)$ & 466(3) & $68(2)$ \\
\hline C(33) & $5277(5)$ & $7875(4)$ & $725(2)$ & $64(2)$ \\
\hline C(34) & 6393(3) & $3755(3)$ & $3684(2)$ & $31(1)$ \\
\hline C(35) & 8080(3) & 3412(3) & $3569(2)$ & $40(2)$ \\
\hline C(36) & $8472(4)$ & $3213(4)$ & 3159(3) & $67(2)$ \\
\hline C(37) & $9053(4)$ & $2670(4)$ & $3200(3)$ & $82(2)$ \\
\hline C(38) & $9230(4)$ & 2324(4) & 3668(3) & $70(2)$ \\
\hline C(39) & $8854(4)$ & 2517(4) & 4083(3) & $70(2)$ \\
\hline$C(40)$ & $8284(4)$ & 3061(3) & 4031(3) & $55(2)$ \\
\hline
\end{tabular}




\begin{tabular}{|c|c|c|c|c|}
\hline$C(41)$ & 7684(3) & $4818(3)$ & $3893(2)$ & $45(2)$ \\
\hline$C(42)$ & $7915(4)$ & $5469(4)$ & 3678(3) & $75(2)$ \\
\hline C(43) & $8275(6)$ & $6013(6)$ & $4003(5)$ & $108(4)$ \\
\hline C(44) & 8388(7) & $5927(6)$ & $4501(6)$ & $125(5)$ \\
\hline$C(45)$ & $8153(5)$ & $5324(5)$ & $4744(4)$ & $96(3)$ \\
\hline$C(46)$ & 7799(4) & $4779(4)$ & 4441(3) & $67(2)$ \\
\hline$C(47)$ & $4805(3)$ & 2925(3) & $4726(2)$ & 28(1) \\
\hline C(48) & $4056(3)$ & $3280(3)$ & $4572(2)$ & $28(1)$ \\
\hline C(49) & $3623(3)$ & $3244(3)$ & $4052(2)$ & 27(1) \\
\hline C(50) & 3976(3) & 2840(3) & $3698(2)$ & $36(2)$ \\
\hline C(51) & 4739(3) & 2490(3) & $3819(2)$ & $32(1)$ \\
\hline C(52) & $5100(4)$ & 2093(3) & $3447(2)$ & $40(2)$ \\
\hline C(53) & $5851(4)$ & 1774(3) & $3578(2)$ & $47(2)$ \\
\hline C(54) & $6298(4)$ & 1852(3) & $4085(2)$ & $52(2)$ \\
\hline$C(55)$ & $5967(3)$ & 2211(3) & $4461(2)$ & $39(2)$ \\
\hline$C(56)$ & 5173(3) & 2552(3) & 4339(2) & $34(1)$ \\
\hline C(57) & 2830(3) & $3648(3)$ & $3888(2)$ & $39(2)$ \\
\hline C(58) & $1133(4)$ & $4046(4)$ & 3920(3) & $52(2)$ \\
\hline C(59) & 569(5) & $4221(5)$ & $4188(4)$ & $110(3)$ \\
\hline C(60) & $-83(6)$ & $4741(5)$ & $4034(4)$ & $105(3)$ \\
\hline C(61) & $-13(6)$ & $5016(5)$ & $3602(5)$ & 131(4) \\
\hline C(62) & $529(6)$ & $4881(5)$ & $3229(5)$ & $140(4)$ \\
\hline C(63) & $1123(6)$ & $4357(5)$ & $3431(4)$ & 111(3) \\
\hline C(64) & 1599(3) & 2539(3) & $3934(2)$ & $39(2)$ \\
\hline C(65) & $1497(4)$ & 1999(4) & 4279(2) & $54(2)$ \\
\hline C(66) & $1208(4)$ & $1321(4)$ & 4085(3) & $60(2)$ \\
\hline C(67) & $1024(4)$ & 1227(3) & $3560(3)$ & $57(2)$ \\
\hline C(68) & $1127(4)$ & $1755(4)$ & $3218(2)$ & $54(2)$ \\
\hline C(69) & $1410(4)$ & 2421(3) & $3410(2)$ & $46(2)$ \\
\hline$C(70)$ & $5107(4)$ & 3920(3) & $283(2)$ & $41(2)$ \\
\hline$C(71)$ & $4668(4)$ & $3526(3)$ & 592(2) & $47(2)$ \\
\hline$C(72)$ & $4866(4)$ & 3513(3) & $1141(2)$ & $40(2)$ \\
\hline C(73) & $5514(4)$ & 3924(3) & $1370(2)$ & $45(2)$ \\
\hline C(74) & $6000(4)$ & 4338(3) & $1077(2)$ & $45(2)$ \\
\hline C(75) & $6675(5)$ & $4764(4)$ & 1311(2) & $65(2)$ \\
\hline C(76) & $7114(5)$ & $5158(4)$ & 1014(3) & $73(2)$ \\
\hline C(77) & $6922(5)$ & $5147(4)$ & $476(3)$ & $70(2)$ \\
\hline C(78) & $6287(4)$ & $4756(4)$ & $238(2)$ & $61(2)$ \\
\hline C(79) & $5801(4)$ & 4340(3) & $530(2)$ & $46(2)$ \\
\hline C(80) & $4323(4)$ & 3102(3) & $1469(2)$ & $56(2)$ \\
\hline C(81) & $5510(4)$ & 1980(3) & 1731(2) & $54(2)$ \\
\hline C(82) & $5973(6)$ & $1457(4)$ & 1511(3) & $92(3)$ \\
\hline C(83) & 6779(8) & 1301(6) & 1737(5) & $124(5)$ \\
\hline C(84) & $7148(7)$ & 1633(7) & $2177(5)$ & $127(5)$ \\
\hline C(85) & $6717(5)$ & 2162(5) & 2403(3) & $86(3)$ \\
\hline
\end{tabular}




\begin{tabular}{|c|c|c|c|c|}
\hline $\mathrm{C}(86)$ & 5891(4) & $2330(4)$ & 2179(3) & $64(2)$ \\
\hline C(87) & $3799(4)$ & $1774(4)$ & $1848(2)$ & $55(2)$ \\
\hline C(88) & 3961(5) & $1120(5)$ & 2050(3) & $76(2)$ \\
\hline C(89) & $3363(5)$ & 739(5) & 2288(3) & $85(3)$ \\
\hline C(90) & $2620(5)$ & 1045(5) & 2336(3) & $82(3)$ \\
\hline $\mathrm{C}(91)$ & 2441(5) & 1688(5) & 2147(3) & 91(3) \\
\hline C(92) & $3028(5)$ & 2053(5) & 1900(3) & $93(3)$ \\
\hline C(93) & 5000 & $993(10)$ & 0 & 201(9) \\
\hline$C(200)$ & $6149(8)$ & 419(6) & 241(5) & $92(4)$ \\
\hline $\mathrm{O}(1)$ & 5295(3) & $5628(2)$ & 2299(2) & $51(1)$ \\
\hline $\mathrm{O}(2)$ & $5584(2)$ & $4029(2)$ & $2645(1)$ & $45(1)$ \\
\hline $\mathrm{O}(3)$ & 5121(3) & $6918(2)$ & 1932(1) & $57(1)$ \\
\hline $\mathrm{O}(4)$ & $7167(2)$ & 4338(2) & 2919(1) & $53(1)$ \\
\hline $\mathrm{O}(5)$ & $3733(2)$ & $3672(2)$ & 4932(1) & $39(1)$ \\
\hline $\mathrm{O}(6)$ & $2120(2)$ & 3393(2) & 4749(2) & $68(1)$ \\
\hline $\mathrm{O}(7)$ & 4025(3) & 3105(3) & $352(2)$ & $62(1)$ \\
\hline $\mathrm{O}(8)$ & 4281(3) & $1914(2)$ & $896(2)$ & $74(1)$ \\
\hline$P(1)$ & 4794(1) & $6819(1)$ & 1366(1) & $50(1)$ \\
\hline $\mathrm{P}(2)$ & 7318(1) & $4110(1)$ & $3474(1)$ & $43(1)$ \\
\hline $\mathrm{P}(3)$ & 1935(1) & 3394(1) & 4173(1) & $51(1)$ \\
\hline $\mathrm{P}(4)$ & $4479(1)$ & 2165(1) & 1447(1) & $54(1)$ \\
\hline C(202) & $4730(20)$ & $10240(20)$ & $450(12)$ & $510(20)$ \\
\hline C(203) & 4428(9) & $9742(8)$ & 813(7) & $187(6)$ \\
\hline C(301) & 4473(19) & 9299(17) & 1718(13) & $414(16)$ \\
\hline C(302) & $5010(16)$ & $9194(14)$ & 2181(10) & 361(13) \\
\hline$C(300)$ & $4670(11)$ & $9672(10)$ & 1204(8) & 237(8) \\
\hline C(303) & $5766(7)$ & 421(8) & $224(6)$ & $79(4)$ \\
\hline
\end{tabular}


Table S3. Bond lengths $[\AA]$ and angles $\left[{ }^{\circ}\right]$ for $\mathbf{1}$.

\begin{tabular}{|c|c|c|c|}
\hline $\mathrm{C}(1)-\mathrm{C}(10)$ & $1.406(7)$ & $\mathrm{C}(35)-\mathrm{C}(40)$ & $1.370(8)$ \\
\hline$C(1)-C(2)$ & $1.406(7)$ & $\mathrm{C}(35)-\mathrm{C}(36)$ & $1.369(8)$ \\
\hline $\mathrm{C}(1)-\mathrm{C}(11)$ & $1.511(7)$ & $\mathrm{C}(35)-\mathrm{P}(2)$ & $1.797(6)$ \\
\hline $\mathrm{C}(2)-\mathrm{O}(1)$ & $1.352(6)$ & $\mathrm{C}(36)-\mathrm{C}(37)$ & $1.383(9)$ \\
\hline $\mathrm{C}(2)-\mathrm{C}(3)$ & $1.423(7)$ & C(37)-C(38) & $1.375(9)$ \\
\hline C(3)-C(4) & $1.393(8)$ & C(38)-C(39) & $1.360(9)$ \\
\hline $\mathrm{C}(3)-\mathrm{C}(21)$ & $1.499(8)$ & C(39)-C(40) & $1.374(8)$ \\
\hline$C(4)-C(5)$ & $1.393(7)$ & $\mathrm{C}(41)-\mathrm{C}(42)$ & $1.425(8)$ \\
\hline C(5)-C(10) & $1.426(7)$ & $\mathrm{C}(41)-\mathrm{C}(46)$ & $1.412(8)$ \\
\hline$C(5)-C(6)$ & $1.436(8)$ & $\mathrm{C}(41)-\mathrm{P}(2)$ & $1.774(6)$ \\
\hline $\mathrm{C}(6)-\mathrm{C}(7)$ & $1.362(8)$ & $\mathrm{C}(42)-\mathrm{C}(43)$ & $1.403(12)$ \\
\hline$C(7)-C(8)$ & $1.413(9)$ & $\mathrm{C}(43)-\mathrm{C}(44)$ & $1.292(13)$ \\
\hline $\mathrm{C}(8)-\mathrm{C}(9)$ & $1.375(8)$ & $\mathrm{C}(44)-\mathrm{C}(45)$ & $1.383(12)$ \\
\hline C(9)-C(10) & $1.416(8)$ & $C(45)-C(46)$ & $1.371(9)$ \\
\hline $\mathrm{C}(11)-\mathrm{C}(12)$ & $1.363(7)$ & $\mathrm{C}(47)-\mathrm{C}(48)$ & $1.389(7)$ \\
\hline $\mathrm{C}(11)-\mathrm{C}(20)$ & $1.440(7)$ & $\mathrm{C}(47)-\mathrm{C}(56)$ & $1.428(7)$ \\
\hline $\mathrm{C}(12)-\mathrm{O}(2)$ & $1.365(6)$ & $\mathrm{C}(47)-\mathrm{C}(47) \# 1$ & $1.470(9)$ \\
\hline $\mathrm{C}(12)-\mathrm{C}(13)$ & $1.409(7)$ & $\mathrm{C}(48)-\mathrm{O}(5)$ & $1.355(5)$ \\
\hline $\mathrm{C}(13)-\mathrm{C}(14)$ & $1.357(6)$ & $\mathrm{C}(48)-\mathrm{C}(49)$ & $1.430(7)$ \\
\hline $\mathrm{C}(13)-\mathrm{C}(34)$ & $1.535(7)$ & $\mathrm{C}(49)-\mathrm{C}(50)$ & $1.380(7)$ \\
\hline$C(14)-C(15)$ & $1.397(7)$ & $\mathrm{C}(49)-\mathrm{C}(57)$ & $1.498(7)$ \\
\hline$C(15)-C(16)$ & $1.394(7)$ & $\mathrm{C}(50)-\mathrm{C}(51)$ & $1.392(7)$ \\
\hline$C(15)-C(20)$ & $1.436(6)$ & $\mathrm{C}(51)-\mathrm{C}(52)$ & $1.414(7)$ \\
\hline $\mathrm{C}(16)-\mathrm{C}(17)$ & $1.375(7)$ & $\mathrm{C}(51)-\mathrm{C}(56)$ & $1.434(7)$ \\
\hline $\mathrm{C}(17)-\mathrm{C}(18)$ & $1.411(7)$ & $\mathrm{C}(52)-\mathrm{C}(53)$ & $1.350(7)$ \\
\hline $\mathrm{C}(18)-\mathrm{C}(19)$ & $1.348(7)$ & $\mathrm{C}(53)-\mathrm{C}(54)$ & $1.414(7)$ \\
\hline $\mathrm{C}(19)-\mathrm{C}(20)$ & $1.395(7)$ & $\mathrm{C}(54)-\mathrm{C}(55)$ & $1.362(7)$ \\
\hline $\mathrm{C}(21)-\mathrm{P}(1)$ & $1.803(6)$ & $\mathrm{C}(55)-\mathrm{C}(56)$ & $1.427(7)$ \\
\hline $\mathrm{C}(22)-\mathrm{C}(27)$ & $1.379(9)$ & $\mathrm{C}(57)-\mathrm{P}(3)$ & $1.781(5)$ \\
\hline$C(22)-C(23)$ & $1.377(8)$ & C(58)-C(59) & $1.267(9)$ \\
\hline $\mathrm{C}(22)-\mathrm{P}(1)$ & $1.795(6)$ & C(58)-C(63) & $1.400(10)$ \\
\hline $\mathrm{C}(23)-\mathrm{C}(24)$ & $1.390(8)$ & C(58)-P(3) & 1.838(7) \\
\hline $\mathrm{C}(24)-\mathrm{C}(25)$ & $1.374(9)$ & $\mathrm{C}(59)-\mathrm{C}(60)$ & $1.453(11)$ \\
\hline$C(25)-C(26)$ & $1.351(10)$ & $\mathrm{C}(60)-\mathrm{C}(61)$ & $1.257(12)$ \\
\hline$C(26)-C(27)$ & $1.384(9)$ & $\mathrm{C}(61)-\mathrm{C}(62)$ & $1.418(12)$ \\
\hline $\mathrm{C}(28)-\mathrm{C}(29)$ & $1.416(8)$ & $\mathrm{C}(62)-\mathrm{C}(63)$ & $1.426(10)$ \\
\hline $\mathrm{C}(28)-\mathrm{C}(33)$ & $1.367(8)$ & $\mathrm{C}(64)-\mathrm{C}(69)$ & $1.372(7)$ \\
\hline $\mathrm{C}(28)-\mathrm{P}(1)$ & $1.791(6)$ & $\mathrm{C}(64)-\mathrm{C}(65)$ & $1.384(8)$ \\
\hline$C(29)-C(30)$ & $1.379(9)$ & $\mathrm{C}(64)-\mathrm{P}(3)$ & $1.790(6)$ \\
\hline $\mathrm{C}(30)-\mathrm{C}(31)$ & $1.365(10)$ & $\mathrm{C}(65)-\mathrm{C}(66)$ & $1.432(9)$ \\
\hline $\mathrm{C}(31)-\mathrm{C}(32)$ & 1.382(9) & $\mathrm{C}(66)-\mathrm{C}(67)$ & $1.366(8)$ \\
\hline $\mathrm{C}(32)-\mathrm{C}(33)$ & $1.407(9)$ & $\mathrm{C}(67)-\mathrm{C}(68)$ & $1.364(8)$ \\
\hline $\mathrm{C}(34)-\mathrm{P}(2)$ & $1.792(5)$ & C(68)-C(69) & $1.408(8)$ \\
\hline
\end{tabular}




\begin{tabular}{|c|c|}
\hline $\mathrm{C}(70)-\mathrm{C}(71)$ & $1.367(7)$ \\
\hline $\mathrm{C}(70)-\mathrm{C}(79)$ & $1.445(8)$ \\
\hline $\mathrm{C}(70)-\mathrm{C}(70) \# 2$ & $1.460(10)$ \\
\hline $\mathrm{C}(71)-\mathrm{O}(7)$ & $1.383(7)$ \\
\hline $\mathrm{C}(71)-\mathrm{C}(72)$ & $1.417(7)$ \\
\hline $\mathrm{C}(72)-\mathrm{C}(73)$ & $1.367(8)$ \\
\hline $\mathrm{C}(72)-\mathrm{C}(80)$ & $1.521(8)$ \\
\hline $\mathrm{C}(73)-\mathrm{C}(74)$ & $1.405(8)$ \\
\hline $\mathrm{C}(74)-\mathrm{C}(79)$ & $1.411(7)$ \\
\hline $\mathrm{C}(74)-\mathrm{C}(75)$ & $1.418(9)$ \\
\hline$C(75)-C(76)$ & $1.345(9)$ \\
\hline $\mathrm{C}(76)-\mathrm{C}(77)$ & $1.388(8)$ \\
\hline $\mathrm{C}(77)-\mathrm{C}(78)$ & $1.340(8)$ \\
\hline $\mathrm{C}(78)-\mathrm{C}(79)$ & $1.408(8)$ \\
\hline $\mathrm{C}(80)-\mathrm{P}(4)$ & $1.795(6)$ \\
\hline $\mathrm{C}(81)-\mathrm{C}(82)$ & $1.410(9)$ \\
\hline $\mathrm{C}(81)-\mathrm{C}(86)$ & $1.402(8)$ \\
\hline $\mathrm{C}(81)-\mathrm{P}(4)$ & $1.753(7)$ \\
\hline C(82)-C(83) & $1.377(12)$ \\
\hline C(83)-C(84) & $1.364(13)$ \\
\hline C(84)-C(85) & $1.396(13)$ \\
\hline $\mathrm{C}(85)-\mathrm{C}(86)$ & $1.410(9)$ \\
\hline$C(10)-C(1)-C(2)$ & 119.1(5) \\
\hline $\mathrm{C}(10)-\mathrm{C}(1)-\mathrm{C}(11)$ & $123.0(5)$ \\
\hline $\mathrm{C}(2)-\mathrm{C}(1)-\mathrm{C}(11)$ & $117.9(5)$ \\
\hline $\mathrm{O}(1)-\mathrm{C}(2)-\mathrm{C}(1)$ & $118.2(5)$ \\
\hline $\mathrm{O}(1)-\mathrm{C}(2)-\mathrm{C}(3)$ & $121.0(5)$ \\
\hline$C(1)-C(2)-C(3)$ & $120.9(5)$ \\
\hline$C(4)-C(3)-C(2)$ & 119.1(5) \\
\hline$C(4)-C(3)-C(21)$ & $120.6(5)$ \\
\hline$C(2)-C(3)-C(21)$ & $120.1(5)$ \\
\hline$C(3)-C(4)-C(5)$ & $121.2(5)$ \\
\hline$C(4)-C(5)-C(10)$ & $119.5(5)$ \\
\hline$C(4)-C(5)-C(6)$ & $121.5(6)$ \\
\hline $\mathrm{C}(10)-\mathrm{C}(5)-\mathrm{C}(6)$ & $118.9(6)$ \\
\hline$C(7)-C(6)-C(5)$ & $119.8(6)$ \\
\hline $\mathrm{C}(6)-\mathrm{C}(7)-\mathrm{C}(8)$ & $121.5(6)$ \\
\hline $\mathrm{C}(9)-\mathrm{C}(8)-\mathrm{C}(7)$ & $119.8(7)$ \\
\hline$C(8)-C(9)-C(10)$ & $120.9(6)$ \\
\hline $\mathrm{C}(1)-\mathrm{C}(10)-\mathrm{C}(9)$ & $120.7(5)$ \\
\hline $\mathrm{C}(1)-\mathrm{C}(10)-\mathrm{C}(5)$ & $120.3(5)$ \\
\hline C(9)-C(10)-C(5) & $119.0(5)$ \\
\hline$C(12)-C(11)-C(20)$ & $119.8(5)$ \\
\hline$C(12)-C(11)-C(1)$ & $120.4(5)$ \\
\hline
\end{tabular}

$\begin{array}{ll}\mathrm{C}(87)-\mathrm{C}(88) & 1.357(9) \\ \mathrm{C}(87)-\mathrm{C}(92) & 1.374(8) \\ \mathrm{C}(87)-\mathrm{P}(4) & 1.778(7) \\ \mathrm{C}(88)-\mathrm{C}(89) & 1.415(9) \\ \mathrm{C}(89)-\mathrm{C}(90) & 1.352(10) \\ \mathrm{C}(90)-\mathrm{C}(91) & 1.328(10) \\ \mathrm{C}(91)-\mathrm{C}(92) & 1.401(10) \\ \mathrm{C}(93)-\mathrm{C}(303) \# 2 & 1.682(16) \\ \mathrm{C}(93)-\mathrm{C}(303) & 1.682(16) \\ \mathrm{C}(93)-\mathrm{C}(200) \# 2 & 2.160(16) \\ \mathrm{C}(200)-\mathrm{C}(303) & 0.613(17) \\ \mathrm{O}(3)-\mathrm{P}(1) & 1.499(4) \\ \mathrm{O}(4)-\mathrm{P}(2) & 1.491(4) \\ \mathrm{O}(6)-\mathrm{P}(3) & 1.482(4) \\ \mathrm{O}(8)-\mathrm{P}(4) & 1.499(4) \\ \mathrm{C}(202)-C(203) & 1.47(3) \\ \mathrm{C}(202)-C(303) \# 3 & 1.85(3) \\ \mathrm{C}(203)-C(300) & 1.04(2) \\ \mathrm{C}(301)-C(300) & 1.58(3) \\ \mathrm{C}(301)-C(302) & 1.39(3) \\ \mathrm{C}(303)-C(202) \# 4 & 1.85(3) \\ & \end{array}$

\begin{tabular}{|c|c|}
\hline$C(20)-C(11)-C(1)$ & $119.7(5)$ \\
\hline $\mathrm{O}(2)-\mathrm{C}(12)-\mathrm{C}(11)$ & $118.3(5)$ \\
\hline $\mathrm{O}(2)-\mathrm{C}(12)-\mathrm{C}(13)$ & $120.7(5)$ \\
\hline$C(11)-C(12)-C(13)$ & $120.9(5)$ \\
\hline$C(14)-C(13)-C(12)$ & $120.1(5)$ \\
\hline$C(14)-C(13)-C(34)$ & $120.2(5)$ \\
\hline$C(12)-C(13)-C(34)$ & $119.7(5)$ \\
\hline$C(13)-C(14)-C(15)$ & $122.1(5)$ \\
\hline$C(14)-C(15)-C(16)$ & 123.1(5) \\
\hline$C(14)-C(15)-C(20)$ & $118.4(5)$ \\
\hline$C(16)-C(15)-C(20)$ & $118.4(5)$ \\
\hline$C(17)-C(16)-C(15)$ & $122.5(5)$ \\
\hline$C(16)-C(17)-C(18)$ & $117.6(5)$ \\
\hline$C(19)-C(18)-C(17)$ & $121.7(5)$ \\
\hline$C(18)-C(19)-C(20)$ & 121.7(6) \\
\hline$C(19)-C(20)-C(15)$ & $118.0(5)$ \\
\hline$C(19)-C(20)-C(11)$ & $123.4(5)$ \\
\hline$C(15)-C(20)-C(11)$ & $118.6(5)$ \\
\hline $\mathrm{C}(3)-\mathrm{C}(21)-\mathrm{P}(1)$ & $114.5(4)$ \\
\hline$C(27)-C(22)-C(23)$ & $118.2(6)$ \\
\hline $\mathrm{C}(27)-\mathrm{C}(22)-\mathrm{P}(1)$ & $120.1(6)$ \\
\hline $\mathrm{C}(23)-\mathrm{C}(22)-\mathrm{P}(1)$ & $121.6(5)$ \\
\hline
\end{tabular}




\begin{tabular}{|c|c|}
\hline$C(22)-C(23)-C(24)$ & $121.5(7)$ \\
\hline$C(25)-C(24)-C(23)$ & $118.0(7)$ \\
\hline$C(26)-C(25)-C(24)$ & $121.9(8)$ \\
\hline$C(25)-C(26)-C(27)$ & $119.4(8)$ \\
\hline$C(22)-C(27)-C(26)$ & $120.9(8)$ \\
\hline $\mathrm{C}(29)-\mathrm{C}(28)-\mathrm{C}(33)$ & $117.5(6)$ \\
\hline C(29)-C(28)-P(1) & $118.9(5)$ \\
\hline C(33)-C(28)-P(1) & $123.3(6)$ \\
\hline C(28)-C(29)-C(30) & $121.2(7)$ \\
\hline C(31)-C(30)-C(29) & $120.2(8)$ \\
\hline C(30)-C(31)-C(32) & $120.1(7)$ \\
\hline C(31)-C(32)-C(33) & $119.6(7)$ \\
\hline C(32)-C(33)-C(28) & $121.3(7)$ \\
\hline $\mathrm{C}(13)-\mathrm{C}(34)-\mathrm{P}(2)$ & 114.9(3) \\
\hline $\mathrm{C}(40)-\mathrm{C}(35)-\mathrm{C}(36)$ & $118.1(6)$ \\
\hline $\mathrm{C}(40)-\mathrm{C}(35)-\mathrm{P}(2)$ & $123.4(4)$ \\
\hline $\mathrm{C}(36)-\mathrm{C}(35)-\mathrm{P}(2)$ & $118.5(5)$ \\
\hline C(35)-C(36)-C(37) & $121.8(7)$ \\
\hline C(38)-C(37)-C(36) & $118.3(6)$ \\
\hline C(39)-C(38)-C(37) & $120.9(6)$ \\
\hline C(38)-C(39)-C(40) & $119.5(6)$ \\
\hline $\mathrm{C}(35)-\mathrm{C}(40)-\mathrm{C}(39)$ & $121.4(6)$ \\
\hline$C(42)-C(41)-C(46)$ & $116.1(6)$ \\
\hline $\mathrm{C}(42)-\mathrm{C}(41)-\mathrm{P}(2)$ & $119.6(6)$ \\
\hline $\mathrm{C}(46)-\mathrm{C}(41)-\mathrm{P}(2)$ & $124.3(5)$ \\
\hline $\mathrm{C}(41)-\mathrm{C}(42)-\mathrm{C}(43)$ & $120.5(8)$ \\
\hline$C(44)-C(43)-C(42)$ & $119.8(11)$ \\
\hline C(43)-C(44)-C(45) & $123.6(13)$ \\
\hline$C(46)-C(45)-C(44)$ & $118.6(9)$ \\
\hline$C(45)-C(46)-C(41)$ & $121.4(7)$ \\
\hline $\mathrm{C}(48)-\mathrm{C}(47)-\mathrm{C}(56)$ & $117.9(5)$ \\
\hline $\mathrm{C}(48)-\mathrm{C}(47)-\mathrm{C}(47) \# 1$ & $120.8(5)$ \\
\hline $\mathrm{C}(56)-\mathrm{C}(47)-\mathrm{C}(47) \# 1$ & $121.3(5)$ \\
\hline $\mathrm{O}(5)-\mathrm{C}(48)-\mathrm{C}(47)$ & $118.0(5)$ \\
\hline $\mathrm{O}(5)-\mathrm{C}(48)-\mathrm{C}(49)$ & 119.6(5) \\
\hline $\mathrm{C}(47)-\mathrm{C}(48)-\mathrm{C}(49)$ & $122.3(5)$ \\
\hline C(50)-C(49)-C(48) & $117.6(5)$ \\
\hline$C(50)-C(49)-C(57)$ & $120.8(5)$ \\
\hline $\mathrm{C}(48)-C(49)-C(57)$ & $121.6(5)$ \\
\hline$C(49)-C(50)-C(51)$ & $123.4(5)$ \\
\hline $\mathrm{C}(50)-\mathrm{C}(51)-\mathrm{C}(52)$ & $122.5(5)$ \\
\hline $\mathrm{C}(50)-\mathrm{C}(51)-\mathrm{C}(56)$ & $117.8(5)$ \\
\hline$C(52)-C(51)-C(56)$ & $119.7(5)$ \\
\hline C(53)-C(52)-C(51) & $120.5(5)$ \\
\hline$C(52)-C(53)-C(54)$ & $120.3(6)$ \\
\hline
\end{tabular}

$\begin{array}{ll}\mathrm{C}(55)-\mathrm{C}(54)-\mathrm{C}(53) & 121.4(6) \\ \mathrm{C}(54)-\mathrm{C}(55)-\mathrm{C}(56) & 119.8(5) \\ \mathrm{C}(47)-\mathrm{C}(56)-\mathrm{C}(55) & 121.1(5) \\ \mathrm{C}(47)-\mathrm{C}(56)-\mathrm{C}(51) & 120.7(5) \\ \mathrm{C}(55)-\mathrm{C}(56)-\mathrm{C}(51) & 118.2(5) \\ \mathrm{C}(49)-\mathrm{C}(57)-\mathrm{P}(3) & 116.9(4) \\ \mathrm{C}(59)-\mathrm{C}(58)-\mathrm{C}(63) & 118.3(8) \\ \mathrm{C}(59)-\mathrm{C}(58)-\mathrm{P}(3) & 120.2(7) \\ \mathrm{C}(63)-\mathrm{C}(58)-\mathrm{P}(3) & 121.5(6) \\ \mathrm{C}(58)-\mathrm{C}(59)-\mathrm{C}(60) & 125.0(9) \\ \mathrm{C}(61)-\mathrm{C}(60)-\mathrm{C}(59) & 111.9(10) \\ \mathrm{C}(60)-\mathrm{C}(61)-\mathrm{C}(62) & 132.8(11) \\ \mathrm{C}(61)-\mathrm{C}(62)-\mathrm{C}(63) & 108.8(10) \\ \mathrm{C}(62)-\mathrm{C}(63)-\mathrm{C}(58) & 123.1(9) \\ \mathrm{C}(69)-\mathrm{C}(64)-\mathrm{C}(65) & 119.6(6) \\ \mathrm{C}(69)-\mathrm{C}(64)-\mathrm{P}(3) & 120.3(5) \\ \mathrm{C}(65)-\mathrm{C}(64)-\mathrm{P}(3) & 120.1(5) \\ \mathrm{C}(66)-\mathrm{C}(65)-\mathrm{C}(64) & 119.8(6) \\ \mathrm{C}(67)-\mathrm{C}(66)-\mathrm{C}(65) & 118.7(6) \\ \mathrm{C}(66)-\mathrm{C}(67)-\mathrm{C}(68) & 121.8(6) \\ \mathrm{C}(69)-\mathrm{C}(68)-\mathrm{C}(67) & 119.3(6) \\ \mathrm{C}(68)-\mathrm{C}(69)-\mathrm{C}(64) & 120.8(6) \\ \mathrm{C}(71)-\mathrm{C}(70)-\mathrm{C}(79) & 118.1(5) \\ \mathrm{C}(71)-\mathrm{C}(70)-\mathrm{C}(70) \# 2 & 121.6(6) \\ \mathrm{C}(79)-\mathrm{C}(70)-\mathrm{C}(70) \# 2 & 120.2(6) \\ \mathrm{O}(7)-\mathrm{C}(71)-\mathrm{C}(70) & 117.9(5) \\ \mathrm{O}(7)-\mathrm{C}(71)-\mathrm{C}(72) & 119.3(5) \\ \mathrm{C}(70)-\mathrm{C}(71)-\mathrm{C}(72) & 122.8(6) \\ \mathrm{C}(73)-\mathrm{C}(72)-\mathrm{C}(71) & 118.3(6) \\ \mathrm{C}(73)-\mathrm{C}(72)-\mathrm{C}(80) & 120.9(5) \\ \mathrm{C}(71)-\mathrm{C}(72)-\mathrm{C}(80) & 120.6(5) \\ \mathrm{C}(72)-\mathrm{C}(73)-\mathrm{C}(74) & 122.2(6) \\ \mathrm{C}(73)-\mathrm{C}(74)-\mathrm{C}(79) & 118.9(6) \\ \mathrm{C}(73)-\mathrm{C}(74)-\mathrm{C}(75) & 122.6(6) \\ \mathrm{C}(79)-\mathrm{C}(74)-\mathrm{C}(75) & 118.5(6) \\ \mathrm{C}(76)-\mathrm{C}(75)-\mathrm{C}(74) & 120.3(6) \\ \mathrm{C}(75)-\mathrm{C}(76)-\mathrm{C}(77) & 120.7(7) \\ \mathrm{C}(76)-\mathrm{C}(77)-\mathrm{C}(78) & 121.1(7) \\ \mathrm{C}(77)-\mathrm{C}(78)-\mathrm{C}(79) & 120.5(6) \\ \mathrm{C}(78)-\mathrm{C}(79)-\mathrm{C}(74) & 118.8(6) \\ \mathrm{C}(78)-\mathrm{C}(79)-\mathrm{C}(70) & 121.5(5) \\ \mathrm{C}(74)-\mathrm{C}(79)-\mathrm{C}(70) & 119.7(6) \\ \mathrm{C}(72)-\mathrm{C}(80)-\mathrm{P}(4) & 113.2(4) \\ \mathrm{C}(82)-\mathrm{C}(81)-\mathrm{C}(4) & 119.4(7) \\ & \end{array}$




\begin{tabular}{|c|c|c|c|}
\hline $\mathrm{C}(86)-\mathrm{C}(81)-\mathrm{P}(4)$ & $121.9(5)$ & $\mathrm{O}(4)-\mathrm{P}(2)-\mathrm{C}(35)$ & $111.0(2)$ \\
\hline $\mathrm{C}(83)-\mathrm{C}(82)-\mathrm{C}(81)$ & $120.0(9)$ & $\mathrm{C}(41)-\mathrm{P}(2)-\mathrm{C}(35)$ & 108.2(3) \\
\hline $\mathrm{C}(82)-\mathrm{C}(83)-\mathrm{C}(84)$ & $122.0(11)$ & $\mathrm{O}(4)-\mathrm{P}(2)-\mathrm{C}(34)$ & $112.2(2)$ \\
\hline $\mathrm{C}(83)-\mathrm{C}(84)-\mathrm{C}(85)$ & 119.9(11) & $\mathrm{C}(41)-\mathrm{P}(2)-\mathrm{C}(34)$ & 108.3(3) \\
\hline $\mathrm{C}(84)-\mathrm{C}(85)-\mathrm{C}(86)$ & 119.1(9) & $\mathrm{C}(35)-\mathrm{P}(2)-\mathrm{C}(34)$ & $105.2(2)$ \\
\hline$C(85)-C(86)-C(81)$ & $120.6(8)$ & $\mathrm{O}(6)-\mathrm{P}(3)-\mathrm{C}(57)$ & $111.3(2)$ \\
\hline $\mathrm{C}(88)-\mathrm{C}(87)-\mathrm{C}(92)$ & $115.9(7)$ & $\mathrm{O}(6)-\mathrm{P}(3)-\mathrm{C}(64)$ & $110.8(3)$ \\
\hline $\mathrm{C}(88)-\mathrm{C}(87)-\mathrm{P}(4)$ & $120.5(6)$ & $\mathrm{C}(57)-\mathrm{P}(3)-\mathrm{C}(64)$ & 108.8(3) \\
\hline $\mathrm{C}(92)-\mathrm{C}(87)-\mathrm{P}(4)$ & $122.7(6)$ & $\mathrm{O}(6)-\mathrm{P}(3)-\mathrm{C}(58)$ & 113.0(3) \\
\hline $\mathrm{C}(87)-\mathrm{C}(88)-\mathrm{C}(89)$ & $122.1(7)$ & $\mathrm{C}(57)-\mathrm{P}(3)-\mathrm{C}(58)$ & 103.8(3) \\
\hline C(90)-C(89)-C(88) & 119.3(8) & $\mathrm{C}(64)-\mathrm{P}(3)-\mathrm{C}(58)$ & 108.8(3) \\
\hline C(91)-C(90)-C(89) & $120.4(8)$ & $\mathrm{O}(8)-\mathrm{P}(4)-\mathrm{C}(81)$ & 113.3(3) \\
\hline $\mathrm{C}(90)-\mathrm{C}(91)-\mathrm{C}(92)$ & 119.8(8) & $\mathrm{O}(8)-\mathrm{P}(4)-\mathrm{C}(80)$ & 109.4(3) \\
\hline $\mathrm{C}(87)-\mathrm{C}(92)-\mathrm{C}(91)$ & $122.4(8)$ & $\mathrm{C}(81)-\mathrm{P}(4)-\mathrm{C}(80)$ & 108.2(3) \\
\hline C(303)\#2-C(93)-C(303) & $99.7(14)$ & $\mathrm{O}(8)-\mathrm{P}(4)-\mathrm{C}(87)$ & 111.3(3) \\
\hline C(303)\#2-C(93)-C(200)\#2 & $211.5(6)$ & $\mathrm{C}(81)-\mathrm{P}(4)-\mathrm{C}(87)$ & 107.3(3) \\
\hline C(303)-C(93)-C(200)\#2 & $109.4(10)$ & $\mathrm{C}(80)-\mathrm{P}(4)-\mathrm{C}(87)$ & 107.0(3) \\
\hline $\mathrm{O}(3)-\mathrm{P}(1)-\mathrm{C}(21)$ & $109.6(2)$ & $\mathrm{C}(203)-\mathrm{C}(202)-\mathrm{C}(303) \# 3$ & $126(3)$ \\
\hline $\mathrm{O}(3)-\mathrm{P}(1)-\mathrm{C}(22)$ & $112.5(3)$ & C(202)-C(203)-C(300) & 126(3) \\
\hline $\mathrm{C}(21)-\mathrm{P}(1)-\mathrm{C}(22)$ & 108.2(3) & C(300)-C(301)-C(302) & $128(3)$ \\
\hline $\mathrm{O}(3)-\mathrm{P}(1)-\mathrm{C}(28)$ & $111.4(3)$ & C(301)-C(300)-C(203) & 142(3) \\
\hline $\mathrm{C}(21)-\mathrm{P}(1)-\mathrm{C}(28)$ & 107.6(3) & C(200)-C(303)-C(93) & 135(3) \\
\hline C(22)-P(1)-C(28) & 107.4(3) & $\mathrm{C}(200)-\mathrm{C}(303)-\mathrm{C}(202) \# 4$ & 111(3) \\
\hline $\mathrm{O}(4)-\mathrm{P}(2)-\mathrm{C}(41)$ & $111.7(3)$ & C(93)-C(303)-C(202)\#4 & $66.2(14)$ \\
\hline
\end{tabular}

Symmetry transformations used to generate equivalent atoms:

$\# 1-\mathrm{x}+1, \mathrm{y},-\mathrm{z}+1 \quad \# 2-\mathrm{x}+1, \mathrm{y},-\mathrm{z} \quad \# 3-\mathrm{x}+1, \mathrm{y}+1,-\mathrm{z}$

$\# 4-\mathrm{x}+1, \mathrm{y}-1,-\mathrm{z}$ 
Table S4. Anisotropic displacement parameters $\left(\AA^{2} \times 10^{3}\right)$ for $\mathbf{1}$. The anisotropic displacement factor exponent takes the form: $-2 \pi^{2}\left[\mathrm{~h}^{2} \mathrm{a}^{* 2} \mathrm{U}^{11}+\ldots+2 \mathrm{hka} * \mathrm{~b} * \mathrm{U}^{12}\right]$

\begin{tabular}{|c|c|c|c|c|c|c|}
\hline & $\mathrm{U}^{11}$ & $\mathrm{U}^{22}$ & $\mathrm{U}^{33}$ & $\mathrm{U}^{23}$ & $\mathrm{U}^{13}$ & $\mathrm{U}^{12}$ \\
\hline$\overline{\mathrm{C}(1)}$ & $37(4)$ & $44(4)$ & $26(3)$ & $4(3)$ & 2(3) & $20(3)$ \\
\hline$C(2)$ & $32(3)$ & $34(4)$ & $34(4)$ & $-9(3)$ & $-6(3)$ & $8(3)$ \\
\hline C(3) & 43(4) & 29(4) & 32(4) & $-4(3)$ & $-7(3)$ & 10(3) \\
\hline C(4) & $50(4)$ & 42(4) & $26(3)$ & $8(3)$ & $5(3)$ & 17(3) \\
\hline C(5) & $52(4)$ & $32(4)$ & $39(4)$ & $-10(3)$ & $-3(3)$ & $0(3)$ \\
\hline C(6) & $66(5)$ & $55(5)$ & $40(4)$ & $-3(3)$ & $5(4)$ & $0(4)$ \\
\hline $\mathrm{C}(7)$ & 76(5) & 93(7) & 50(5) & $-28(4)$ & $-6(4)$ & $-28(5)$ \\
\hline$C(8)$ & $92(6)$ & 82(6) & $55(5)$ & $-14(4)$ & $15(4)$ & $-41(5)$ \\
\hline C(9) & $66(4)$ & 66(5) & 41(4) & $-8(4)$ & $9(3)$ & $-18(4)$ \\
\hline$C(10)$ & $55(4)$ & $26(3)$ & 31(3) & $-3(3)$ & 5(3) & $-5(3)$ \\
\hline $\mathrm{C}(11)$ & $37(3)$ & 29(3) & $38(4)$ & $0(3)$ & 6(3) & $0(3)$ \\
\hline$C(12)$ & $33(3)$ & $32(4)$ & 32(3) & 13(3) & $-3(3)$ & $9(3)$ \\
\hline C(13) & $34(3)$ & 25(3) & $37(3)$ & $10(3)$ & 2(3) & $-3(3)$ \\
\hline C(14) & $26(3)$ & 28(3) & 30(3) & $5(3)$ & $-1(3)$ & $1(3)$ \\
\hline C(15) & $30(3)$ & 33(3) & 23(3) & $5(3)$ & $0(3)$ & $-11(3)$ \\
\hline$C(16)$ & $37(4)$ & $33(4)$ & $36(4)$ & $-11(3)$ & 4(3) & $-1(3)$ \\
\hline C(17) & $53(4)$ & $43(4)$ & $32(4)$ & $-7(3)$ & $9(3)$ & $3(3)$ \\
\hline C(18) & $45(4)$ & $47(4)$ & 42(4) & $-2(3)$ & $3(3)$ & 25(3) \\
\hline C(19) & $43(4)$ & $50(4)$ & $37(4)$ & $4(3)$ & $-1(3)$ & $10(3)$ \\
\hline$C(20)$ & 28(3) & $38(4)$ & 30(3) & 3(3) & $-2(3)$ & $4(3)$ \\
\hline$C(21)$ & $51(4)$ & $68(5)$ & $26(3)$ & $12(3)$ & 2(3) & 27(3) \\
\hline$C(22)$ & $68(5)$ & 49(4) & $40(4)$ & 11(3) & $17(4)$ & $22(4)$ \\
\hline C(23) & $52(5)$ & 79(6) & 72(5) & $37(4)$ & $12(4)$ & $28(4)$ \\
\hline$C(24)$ & $70(5)$ & $77(6)$ & 76(6) & 21(5) & $12(4)$ & $9(5)$ \\
\hline C(25) & $79(6)$ & $65(6)$ & 101(7) & $30(5)$ & $41(6)$ & $28(5)$ \\
\hline$C(26)$ & $92(7)$ & $86(7)$ & $74(6)$ & $5(5)$ & $8(5)$ & $33(5)$ \\
\hline$C(27)$ & $84(6)$ & 99(7) & 56(5) & 12(5) & 18(5) & $22(5)$ \\
\hline C(28) & $56(4)$ & 58(5) & $52(4)$ & $-3(4)$ & 4(3) & $-11(4)$ \\
\hline C(29) & 68(5) & 74(6) & $97(6)$ & $15(4)$ & $31(5)$ & $18(5)$ \\
\hline C(30) & $78(6)$ & 89(7) & $111(7)$ & $17(6)$ & $49(5)$ & $9(5)$ \\
\hline$C(31)$ & 83(6) & 82(7) & 55(5) & $-8(4)$ & $17(4)$ & $-11(5)$ \\
\hline C(32) & $75(5)$ & $70(6)$ & $55(5)$ & $1(4)$ & $-8(4)$ & $-22(5)$ \\
\hline C(33) & $72(5)$ & $64(6)$ & $49(4)$ & $13(4)$ & $-13(4)$ & $8(4)$ \\
\hline C(34) & $36(3)$ & 15(3) & 41(3) & $8(3)$ & 1(3) & $9(3)$ \\
\hline C(35) & $31(3)$ & $43(4)$ & $46(4)$ & 15(3) & $10(3)$ & $8(3)$ \\
\hline$C(36)$ & $52(4)$ & $95(6)$ & 55(5) & $-3(4)$ & $10(4)$ & $18(4)$ \\
\hline C(37) & $62(5)$ & $110(7)$ & 76(6) & $3(5)$ & $16(4)$ & $52(5)$ \\
\hline C(38) & $41(4)$ & $59(5)$ & $111(7)$ & $5(5)$ & $18(5)$ & $25(4)$ \\
\hline C(39) & $56(5)$ & 65(5) & $94(6)$ & $38(4)$ & $26(4)$ & $30(4)$ \\
\hline C(40) & $45(4)$ & 56(5) & 67(5) & 1(4) & $19(4)$ & $18(4)$ \\
\hline
\end{tabular}




\begin{tabular}{|c|c|c|c|c|c|c|}
\hline$C(41)$ & $37(4)$ & $35(4)$ & $64(4)$ & $12(3)$ & $13(3)$ & $10(3)$ \\
\hline$C(42)$ & $36(4)$ & $60(6)$ & 132(7) & $26(5)$ & $28(4)$ & $7(4)$ \\
\hline$C(43)$ & $65(6)$ & $49(6)$ & $214(14)$ & 19(9) & $39(9)$ & $-5(5)$ \\
\hline$C(44)$ & $87(7)$ & $40(7)$ & $257(16)$ & $-26(9)$ & 61(11) & $-6(5)$ \\
\hline$C(45)$ & $85(6)$ & $95(8)$ & $110(7)$ & $-59(6)$ & $22(5)$ & $-3(6)$ \\
\hline$C(46)$ & $69(5)$ & $55(5)$ & $78(5)$ & $-3(4)$ & $17(4)$ & $-15(4)$ \\
\hline$C(47)$ & 31(3) & $27(3)$ & $27(3)$ & $-1(2)$ & 11(3) & $-3(3)$ \\
\hline$C(48)$ & $28(3)$ & $27(3)$ & $30(3)$ & $-8(3)$ & $8(3)$ & $-9(3)$ \\
\hline$C(49)$ & $27(3)$ & $22(3)$ & $32(3)$ & $-3(3)$ & $4(3)$ & $-1(3)$ \\
\hline$C(50)$ & $31(3)$ & $36(4)$ & $39(4)$ & $-2(3)$ & $1(3)$ & $-14(3)$ \\
\hline$C(51)$ & $38(4)$ & $30(4)$ & $29(3)$ & $2(3)$ & $5(3)$ & $3(3)$ \\
\hline$C(52)$ & $51(4)$ & $40(4)$ & $28(3)$ & $-2(3)$ & $0(3)$ & $-2(3)$ \\
\hline$C(53)$ & $60(4)$ & $36(4)$ & $45(4)$ & $-7(3)$ & $10(3)$ & $10(3)$ \\
\hline$C(54)$ & $57(4)$ & $47(4)$ & $53(4)$ & $19(3)$ & $13(4)$ & $23(3)$ \\
\hline$C(55)$ & $39(4)$ & $47(4)$ & $29(3)$ & $4(3)$ & $3(3)$ & $19(3)$ \\
\hline$C(56)$ & $34(3)$ & $34(4)$ & $34(3)$ & $0(3)$ & $10(3)$ & $-4(3)$ \\
\hline$C(57)$ & $35(3)$ & $36(4)$ & $42(4)$ & $-12(3)$ & $-10(3)$ & $2(3)$ \\
\hline$C(58)$ & $31(4)$ & $57(5)$ & $67(5)$ & $1(4)$ & $8(4)$ & $3(3)$ \\
\hline$C(59)$ & $70(6)$ & $144(9)$ & $114(7)$ & $-39(6)$ & $6(6)$ & $18(7)$ \\
\hline$C(60)$ & $58(6)$ & $99(8)$ & $159(10)$ & $26(7)$ & $23(6)$ & $5(6)$ \\
\hline$C(61)$ & $84(7)$ & $79(8)$ & 251(14) & $-3(9)$ & $92(9)$ & $14(6)$ \\
\hline$C(62)$ & $98(8)$ & $110(9)$ & $213(12)$ & $54(8)$ & $29(8)$ & $45(7)$ \\
\hline$C(63)$ & $98(7)$ & $80(7)$ & 153(9) & $5(6)$ & $17(6)$ & $35(6)$ \\
\hline$C(64)$ & $26(3)$ & $45(4)$ & $45(4)$ & $-3(3)$ & $6(3)$ & $0(3)$ \\
\hline$C(65)$ & $47(4)$ & $60(5)$ & $50(4)$ & $12(4)$ & $-6(3)$ & $3(4)$ \\
\hline$C(66)$ & $46(4)$ & $45(5)$ & $87(6)$ & $24(4)$ & $2(4)$ & $14(4)$ \\
\hline$C(67)$ & $47(4)$ & $26(4)$ & $100(6)$ & $-8(4)$ & $15(4)$ & $6(3)$ \\
\hline$C(68)$ & $55(4)$ & $47(5)$ & $64(4)$ & $-10(4)$ & $22(4)$ & $-4(4)$ \\
\hline $\mathrm{C}(69)$ & $53(4)$ & $41(4)$ & $48(4)$ & $-2(3)$ & $17(3)$ & $-13(3)$ \\
\hline$C(70)$ & $54(4)$ & $39(4)$ & $28(3)$ & $-4(3)$ & $-3(3)$ & $6(3)$ \\
\hline $\mathrm{C}(71)$ & $47(4)$ & $54(5)$ & $38(4)$ & $0(3)$ & $7(3)$ & $7(3)$ \\
\hline$C(72)$ & $38(4)$ & $43(4)$ & $35(4)$ & $-7(3)$ & $-4(3)$ & $10(3)$ \\
\hline C(73) & $71(5)$ & $33(4)$ & $32(4)$ & $6(3)$ & $5(3)$ & $35(4)$ \\
\hline$C(74)$ & $55(4)$ & $35(4)$ & $38(4)$ & $-6(3)$ & $-13(3)$ & $6(3)$ \\
\hline$C(75)$ & $105(6)$ & $44(5)$ & $36(4)$ & $3(4)$ & $-23(4)$ & $13(4)$ \\
\hline$C(76)$ & $96(6)$ & $52(5)$ & $61(5)$ & $7(4)$ & $-23(5)$ & $-12(4)$ \\
\hline$C(77)$ & $82(6)$ & $54(5)$ & $67(5)$ & $10(4)$ & $-13(5)$ & $-24(4)$ \\
\hline$C(78)$ & $73(5)$ & $64(5)$ & $39(4)$ & $8(4)$ & $-13(4)$ & $-12(4)$ \\
\hline$C(79)$ & $57(4)$ & $44(4)$ & $35(4)$ & $-2(3)$ & $-2(3)$ & $6(3)$ \\
\hline$C(80)$ & $51(4)$ & $73(5)$ & $44(4)$ & $-7(3)$ & $12(3)$ & $-1(4)$ \\
\hline $\mathrm{C}(81)$ & $65(5)$ & $50(5)$ & $52(4)$ & $6(3)$ & $23(4)$ & $3(4)$ \\
\hline$C(82)$ & $94(7)$ & $69(6)$ & $126(7)$ & $-1(5)$ & $59(6)$ & $16(5)$ \\
\hline$C(83)$ & $113(11)$ & $77(8)$ & $205(14)$ & $40(8)$ & $99(10)$ & $47(7)$ \\
\hline$C(84)$ & $66(7)$ & $126(12)$ & 195(13) & 117(10) & 38(8) & $45(7)$ \\
\hline$C(85)$ & $65(6)$ & 111(7) & $84(6)$ & $41(5)$ & $18(5)$ & $11(5)$ \\
\hline
\end{tabular}




\begin{tabular}{lcccccc}
$\mathrm{C}(86)$ & $51(5)$ & $71(5)$ & $66(5)$ & $31(4)$ & $1(4)$ & $14(4)$ \\
$\mathrm{C}(87)$ & $52(4)$ & $73(5)$ & $38(4)$ & $3(4)$ & $-1(3)$ & $11(4)$ \\
$\mathrm{C}(88)$ & $67(5)$ & $94(7)$ & $66(5)$ & $8(5)$ & $12(4)$ & $1(5)$ \\
$\mathrm{C}(89)$ & $77(6)$ & $102(7)$ & $77(6)$ & $22(5)$ & $15(5)$ & $-23(5)$ \\
$\mathrm{C}(90)$ & $56(6)$ & $120(8)$ & $70(6)$ & $18(5)$ & $5(4)$ & $-17(5)$ \\
$\mathrm{C}(91)$ & $66(6)$ & $124(8)$ & $95(6)$ & $27(6)$ & $47(5)$ & $16(6)$ \\
$\mathrm{C}(92)$ & $73(6)$ & $100(7)$ & $113(7)$ & $30(5)$ & $38(5)$ & $16(5)$ \\
$\mathrm{C}(93)$ & $350(30)$ & $161(17)$ & $97(12)$ & 0 & $49(15)$ & 0 \\
$\mathrm{C}(200)$ & $104(10)$ & $86(7)$ & $91(8)$ & $18(5)$ & $34(8)$ & $-14(8)$ \\
$\mathrm{O}(1)$ & $53(3)$ & $61(3)$ & $36(3)$ & $18(2)$ & $-3(2)$ & $3(2)$ \\
$\mathrm{O}(2)$ & $47(2)$ & $52(3)$ & $36(2)$ & $-4(2)$ & $12(2)$ & $17(2)$ \\
$\mathrm{O}(3)$ & $84(3)$ & $55(3)$ & $30(2)$ & $7(2)$ & $-1(2)$ & $8(2)$ \\
$\mathrm{O}(4)$ & $44(2)$ & $71(3)$ & $48(3)$ & $17(2)$ & $16(2)$ & $13(2)$ \\
$\mathrm{O}(5)$ & $35(2)$ & $41(2)$ & $42(2)$ & $-6(2)$ & $8(2)$ & $0(2)$ \\
$\mathrm{O}(6)$ & $44(3)$ & $107(4)$ & $56(3)$ & $-35(3)$ & $19(2)$ & $-30(3)$ \\
$\mathrm{O}(7)$ & $51(3)$ & $91(4)$ & $41(3)$ & $4(2)$ & $0(2)$ & $-14(3)$ \\
$\mathrm{O}(8)$ & $107(4)$ & $70(4)$ & $47(3)$ & $-12(2)$ & $19(3)$ & $-16(3)$ \\
$\mathrm{P}(1)$ & $61(1)$ & $48(1)$ & $41(1)$ & $9(1)$ & $7(1)$ & $13(1)$ \\
$\mathrm{P}(2)$ & $39(1)$ & $45(1)$ & $47(1)$ & $12(1)$ & $13(1)$ & $8(1)$ \\
$\mathrm{P}(3)$ & $35(1)$ & $60(1)$ & $59(1)$ & $-24(1)$ & $7(1)$ & $-5(1)$ \\
$\mathrm{P}(4)$ & $65(1)$ & $55(1)$ & $44(1)$ & $-2(1)$ & $14(1)$ & $5(1)$ \\
& & & & & & \\
\hline
\end{tabular}


Table S5. Hydrogen coordinates $\left(\times 10^{4}\right)$ and isotropic displacement parameters $\left(\AA^{2} \times 10^{3}\right)$ for $\mathbf{1}$.

\begin{tabular}{|c|c|c|c|c|}
\hline & $\mathrm{x}$ & $\mathrm{y}$ & $\mathrm{z}$ & $\mathrm{U}(\mathrm{eq})$ \\
\hline $\mathrm{H}(4)$ & 3310 & 5214 & 971 & 47 \\
\hline $\mathrm{H}(6)$ & 2091 & 4433 & 1042 & 65 \\
\hline $\mathrm{H}(7)$ & 1452 & 3643 & 1519 & 90 \\
\hline $\mathrm{H}(8)$ & 1994 & 3398 & 2383 & 91 \\
\hline $\mathrm{H}(9)$ & 3202 & 3946 & 2765 & 69 \\
\hline $\mathrm{H}(14)$ & 5485 & 4410 & 4300 & 34 \\
\hline $\mathrm{H}(16)$ & 4372 & 5120 & 4609 & 43 \\
\hline $\mathrm{H}(17)$ & 3182 & 5806 & 4442 & 51 \\
\hline $\mathrm{H}(18)$ & 2571 & 5969 & 3575 & 54 \\
\hline H(19) & 3149 & 5499 & 2913 & 52 \\
\hline $\mathrm{H}(21 \mathrm{~A})$ & 4524 & 5833 & 850 & 59 \\
\hline $\mathrm{H}(21 \mathrm{~B})$ & 5331 & 5711 & 1253 & 59 \\
\hline $\mathrm{H}(23)$ & 3467 & 6663 & 514 & 81 \\
\hline $\mathrm{H}(24)$ & 2116 & 7107 & 303 & 89 \\
\hline $\mathrm{H}(25)$ & 1671 & 7972 & 825 & 95 \\
\hline $\mathrm{H}(26)$ & 2458 & 8283 & 1588 & 101 \\
\hline $\mathrm{H}(27)$ & 3776 & 7798 & 1817 & 95 \\
\hline H(29) & 6394 & 6504 & 1084 & 94 \\
\hline $\mathrm{H}(30)$ & 7324 & 7053 & 621 & 107 \\
\hline $\mathrm{H}(31)$ & 7039 & 8163 & 288 & 87 \\
\hline $\mathrm{H}(32)$ & 5734 & 8664 & 310 & 82 \\
\hline H(33) & 4755 & 8079 & 734 & 77 \\
\hline $\mathrm{H}(34 \mathrm{~A})$ & 6276 & 3299 & 3520 & 37 \\
\hline $\mathrm{H}(34 \mathrm{~B})$ & 6506 & 3678 & 4057 & 37 \\
\hline H(36) & 8345 & 3449 & 2843 & 80 \\
\hline $\mathrm{H}(37)$ & 9318 & 2543 & 2919 & 99 \\
\hline H(38) & 9611 & 1952 & 3701 & 84 \\
\hline H(39) & 8982 & 2282 & 4399 & 84 \\
\hline $\mathrm{H}(40)$ & 8031 & 3194 & 4316 & 66 \\
\hline $\mathrm{H}(42)$ & 7827 & 5533 & 3319 & 89 \\
\hline $\mathrm{H}(43)$ & 8433 & 6434 & 3860 & 129 \\
\hline $\mathrm{H}(44)$ & 8642 & 6290 & 4708 & 149 \\
\hline $\mathrm{H}(45)$ & 8233 & 5290 & 5104 & 115 \\
\hline $\mathrm{H}(46)$ & 7631 & 4374 & 4601 & 80 \\
\hline $\mathrm{H}(50)$ & 3689 & 2799 & 3362 & 43 \\
\hline $\mathrm{H}(52)$ & 4819 & 2051 & 3110 & 48 \\
\hline $\mathrm{H}(53)$ & 6074 & 1501 & 3334 & 56 \\
\hline $\mathrm{H}(54)$ & 6829 & 1653 & 4162 & 62 \\
\hline $\mathrm{H}(55)$ & 6258 & 2234 & 4797 & 46 \\
\hline $\mathrm{H}(57 \mathrm{~A})$ & 2689 & 3607 & 3513 & 47 \\
\hline H(57B) & 2941 & 4143 & 3966 & 47 \\
\hline
\end{tabular}




\begin{tabular}{|c|c|c|c|c|}
\hline H(59) & 572 & 4000 & 4508 & 132 \\
\hline $\mathrm{H}(60)$ & -502 & 4856 & 4231 & 126 \\
\hline $\mathrm{H}(61)$ & -392 & 5380 & 3509 & 157 \\
\hline $\mathrm{H}(62)$ & 500 & 5099 & 2906 & 168 \\
\hline H(63) & 1523 & 4215 & 3227 & 133 \\
\hline H(65) & 1616 & 2077 & 4636 & 65 \\
\hline $\mathrm{H}(66)$ & 1146 & 952 & 4313 & 72 \\
\hline $\mathrm{H}(67)$ & 822 & 792 & 3432 & 69 \\
\hline H(68) & 1012 & 1675 & 2861 & 65 \\
\hline H(69) & 1469 & 2785 & 3178 & 55 \\
\hline $\mathrm{H}(73)$ & 5638 & 3930 & 1730 & 55 \\
\hline $\mathrm{H}(75)$ & 6814 & 4772 & 1671 & 78 \\
\hline H(76) & 7550 & 5441 & 1171 & 88 \\
\hline $\mathrm{H}(77)$ & 7241 & 5415 & 278 & 84 \\
\hline H(78) & 6166 & 4759 & -123 & 73 \\
\hline $\mathrm{H}(80 \mathrm{~A})$ & 3738 & 3206 & 1348 & 67 \\
\hline H(80B) & 4447 & 3260 & 1827 & 67 \\
\hline $\mathrm{H}(82)$ & 5735 & 1217 & 1214 & 110 \\
\hline H(83) & 7079 & 959 & 1585 & 149 \\
\hline $\mathrm{H}(84)$ & 7688 & 1507 & 2327 & 153 \\
\hline $\mathrm{H}(85)$ & 6971 & 2400 & 2697 & 103 \\
\hline H(86) & 5596 & 2676 & 2330 & 76 \\
\hline H(88) & 4480 & 916 & 2032 & 91 \\
\hline H(89) & 3480 & 283 & 2410 & 102 \\
\hline $\mathrm{H}(90)$ & 2232 & 804 & 2502 & 99 \\
\hline $\mathrm{H}(91)$ & 1927 & 1894 & 2179 & 110 \\
\hline H(92) & 2892 & 2501 & 1767 & 112 \\
\hline $\mathrm{H}(1)$ & 5339 & 6021 & 2175 & 77 \\
\hline $\mathrm{H}(2)$ & 6096 & 4062 & 2703 & 67 \\
\hline $\mathrm{H}(5)$ & 3233 & 3582 & 4919 & 59 \\
\hline $\mathrm{H}(7 \mathrm{~A})$ & 3648 & 3088 & 532 & 92 \\
\hline
\end{tabular}


Table S6. Crystal data and structure refinement for 2.

Identification code

Empirical formula

Formula weight

Temperature

Wavelength

Crystal system

Space group

Unit cell dimensions

Volume

Z

Density (calculated)

Absorption coefficient

$\mathrm{F}(000)$

Crystal size

Theta range for data collection

Index ranges

Reflections collected

Independent reflections

Completeness to theta $=28.78^{\circ}$

Absorption correction

Max. and min. transmission

Refinement method

Data / restraints / parameters

Goodness-of-fit on $\mathrm{F}^{2}$

Final R indices [I $>2 \operatorname{sigma(I)]~}$

$\mathrm{R}$ indices (all data)

Absolute structure parameter

Largest diff. peak and hole
2 (bpe, BINOL-P(O)Et ${ }_{2}$ )

C30 H36 O4 P2

522.53

173(2) K

$0.71073 \AA$

Monoclinic

P21

$\mathrm{a}=8.7568(9) \AA \quad \alpha=90^{\circ}$.

$\mathrm{b}=17.2784(17) \AA \quad \beta=104.529(2)^{\circ}$.

$\mathrm{c}=9.5484(10) \AA \quad \gamma=90^{\circ}$.

$1398.5(2) \AA^{3}$

2

$1.241 \mathrm{~g} / \mathrm{cm}^{3}$

$0.188 \mathrm{~mm}^{-1}$

556

$0.40 \times 0.35 \times 0.10 \mathrm{~mm}^{3}$

2.20 to $28.78^{\circ}$

$-11 \leq \mathrm{h} \leq 11,-22 \leq \mathrm{k} \leq 22,-12 \leq \mathrm{l} \leq 12$

12937

$6346[\mathrm{R}(\mathrm{int})=0.0263]$

$93.2 \%$

Semi-empirical from equivalents

0.9814 and 0.9284

Full-matrix least-squares on $\mathrm{F}^{2}$

6346 / 1 / 331

1.046

$\mathrm{R} 1=0.0508, \mathrm{wR} 2=0.1241$

$\mathrm{R} 1=0.0695, \mathrm{wR} 2=0.1369$

0.01(10)

0.744 and -0.253 e. $\AA^{-3}$ 
Table S7. Atomic coordinates $\left(\times 10^{4}\right)$ and equivalent isotropic displacement parameters $\left(\AA^{2} \times\right.$ $10^{3}$ ) for 2 . $U(e q)$ is defined as one third of the trace of the orthogonalized $U^{i j}$ tensor.

\begin{tabular}{|c|c|c|c|c|}
\hline & $\mathrm{X}$ & $\mathrm{y}$ & $\mathrm{z}$ & $\mathrm{U}(\mathrm{eq})$ \\
\hline $\mathrm{C}(1)$ & $930(3)$ & $3342(2)$ & $7842(3)$ & $27(1)$ \\
\hline $\mathrm{C}(2)$ & 1477(3) & $3932(2)$ & $7150(3)$ & $27(1)$ \\
\hline C(3) & $439(3)$ & $4387(2)$ & $6066(3)$ & $30(1)$ \\
\hline C(4) & $-1143(3)$ & $4262(2)$ & 5790(3) & $35(1)$ \\
\hline C(5) & $-1778(3)$ & $3685(2)$ & $6534(3)$ & $37(1)$ \\
\hline C(6) & $-3438(4)$ & $3577(2)$ & $6296(4)$ & $52(1)$ \\
\hline C(7) & $-4025(4)$ & $3043(2)$ & $7065(5)$ & $56(1)$ \\
\hline C(8) & $-2983(4)$ & $2586(2)$ & $8117(4)$ & $48(1)$ \\
\hline C(9) & $-1387(4)$ & 2663(2) & 8332(3) & $37(1)$ \\
\hline$C(10)$ & $-729(3)$ & $3211(2)$ & 7564(3) & $30(1)$ \\
\hline $\mathrm{C}(11)$ & 2035(3) & $2868(2)$ & 8967(3) & $26(1)$ \\
\hline$C(12)$ & 2709(3) & $2205(2)$ & 8619(3) & $27(1)$ \\
\hline C(13) & 3739(3) & $1749(2)$ & $9706(3)$ & $28(1)$ \\
\hline C(14) & 3966(3) & $1954(2)$ & 11118(3) & $30(1)$ \\
\hline C(15) & 3222(3) & $2600(2)$ & 11544(3) & $32(1)$ \\
\hline$C(16)$ & 3392(4) & 2799(2) & 13024(3) & $47(1)$ \\
\hline $\mathrm{C}(17)$ & $2629(5)$ & $3410(2)$ & 13399(4) & $58(1)$ \\
\hline C(18) & $1680(5)$ & $3889(2)$ & $12314(4)$ & $57(1)$ \\
\hline C(19) & $1522(4)$ & $3728(2)$ & 10890(3) & $40(1)$ \\
\hline$C(20)$ & 2275(3) & $3078(2)$ & 10453(3) & $30(1)$ \\
\hline$C(21)$ & 1087(3) & $4978(2)$ & $5205(3)$ & $31(1)$ \\
\hline C(22) & $2717(4)$ & $6413(2)$ & 4992(4) & $49(1)$ \\
\hline C(23) & 4146(5) & 6031(3) & $4639(4)$ & $62(1)$ \\
\hline C(24) & $254(4)$ & $6366(2)$ & $6527(4)$ & $50(1)$ \\
\hline$C(25)$ & $785(6)$ & 7035(3) & $7568(6)$ & $84(2)$ \\
\hline$C(26)$ & 4512(3) & $1026(2)$ & 9314(3) & $29(1)$ \\
\hline $\mathrm{C}(27)$ & 2153(6) & $67(3)$ & $10224(6)$ & $76(1)$ \\
\hline C(28) & $718(7)$ & $540(3)$ & 10181(8) & $108(2)$ \\
\hline C(29) & $4241(4)$ & $-603(2)$ & $8650(4)$ & $49(1)$ \\
\hline$C(30)$ & $3201(5)$ & $-1319(2)$ & $8177(4)$ & $54(1)$ \\
\hline $\mathrm{O}(1)$ & $3064(2)$ & 4067(1) & $7479(2)$ & $34(1)$ \\
\hline $\mathrm{O}(2)$ & 2416(3) & 1997(1) & $7186(2)$ & $39(1)$ \\
\hline $\mathrm{O}(3)$ & 3117(3) & $5616(1)$ & 7551(2) & $40(1)$ \\
\hline $\mathrm{O}(4)$ & 1979(3) & $449(2)$ & $7360(3)$ & $53(1)$ \\
\hline$P(1)$ & 1894(1) & 5834(1) & 6203(1) & $32(1)$ \\
\hline $\mathrm{P}(2)$ & $3117(1)$ & 243(1) & 8752(1) & $35(1)$ \\
\hline
\end{tabular}


Table S8. Bond lengths $[\AA]$ and angles $\left[{ }^{\circ}\right]$ for 2.

\begin{tabular}{|c|c|c|c|}
\hline $\mathrm{C}(1)-\mathrm{C}(2)$ & $1.365(4)$ & $\mathrm{C}(14)-\mathrm{C}(15)$ & $1.403(4)$ \\
\hline $\mathrm{C}(1)-\mathrm{C}(10)$ & $1.428(4)$ & $C(15)-C(20)$ & $1.423(4)$ \\
\hline $\mathrm{C}(1)-\mathrm{C}(11)$ & $1.496(4)$ & $\mathrm{C}(15)-\mathrm{C}(16)$ & $1.426(4)$ \\
\hline $\mathrm{C}(2)-\mathrm{O}(1)$ & $1.366(3)$ & $\mathrm{C}(16)-\mathrm{C}(17)$ & $1.346(5)$ \\
\hline $\mathrm{C}(2)-\mathrm{C}(3)$ & $1.429(4)$ & $\mathrm{C}(17)-\mathrm{C}(18)$ & $1.420(5)$ \\
\hline$C(3)-C(4)$ & $1.361(4)$ & C(18)-C(19) & $1.360(4)$ \\
\hline$C(3)-C(21)$ & $1.508(4)$ & C(19)-C(20) & $1.416(4)$ \\
\hline$C(4)-C(5)$ & $1.416(5)$ & $\mathrm{C}(21)-\mathrm{P}(1)$ & $1.805(3)$ \\
\hline$C(5)-C(10)$ & $1.424(4)$ & $\mathrm{C}(22)-\mathrm{C}(23)$ & $1.525(6)$ \\
\hline$C(5)-C(6)$ & $1.427(5)$ & $\mathrm{C}(22)-\mathrm{P}(1)$ & $1.809(3)$ \\
\hline$C(6)-C(7)$ & $1.358(6)$ & $\mathrm{C}(24)-\mathrm{C}(25)$ & $1.519(5)$ \\
\hline$C(7)-C(8)$ & $1.416(6)$ & $\mathrm{C}(24)-\mathrm{P}(1)$ & $1.796(4)$ \\
\hline $\mathrm{C}(8)-\mathrm{C}(9)$ & $1.368(5)$ & $\mathrm{C}(26)-\mathrm{P}(2)$ & $1.810(3)$ \\
\hline $\mathrm{C}(9)-\mathrm{C}(10)$ & $1.406(4)$ & $\mathrm{C}(27)-\mathrm{C}(28)$ & $1.492(7)$ \\
\hline $\mathrm{C}(11)-\mathrm{C}(12)$ & $1.368(4)$ & $\mathrm{C}(27)-\mathrm{P}(2)$ & $1.837(4)$ \\
\hline $\mathrm{C}(11)-\mathrm{C}(20)$ & $1.428(4)$ & C(29)-C(30) & $1.536(5)$ \\
\hline $\mathrm{C}(12)-\mathrm{O}(2)$ & $1.375(3)$ & $\mathrm{C}(29)-\mathrm{P}(2)$ & $1.779(3)$ \\
\hline$C(12)-C(13)$ & $1.429(4)$ & $\mathrm{O}(3)-\mathrm{P}(1)$ & $1.501(2)$ \\
\hline $\mathrm{C}(13)-\mathrm{C}(14)$ & $1.360(4)$ & $\mathrm{O}(4)-\mathrm{P}(2)$ & $1.491(2)$ \\
\hline $\mathrm{C}(13)-\mathrm{C}(26)$ & $1.512(4)$ & & \\
\hline $\mathrm{C}(2)-\mathrm{C}(1)-\mathrm{C}(10)$ & $119.5(2)$ & $\mathrm{C}(20)-\mathrm{C}(11)-\mathrm{C}(1)$ & $118.8(2)$ \\
\hline $\mathrm{C}(2)-\mathrm{C}(1)-\mathrm{C}(11)$ & $120.9(2)$ & $\mathrm{O}(2)-\mathrm{C}(12)-\mathrm{C}(11)$ & $118.4(2)$ \\
\hline $\mathrm{C}(10)-\mathrm{C}(1)-\mathrm{C}(11)$ & $119.4(2)$ & $\mathrm{O}(2)-\mathrm{C}(12)-\mathrm{C}(13)$ & $120.3(2)$ \\
\hline $\mathrm{O}(1)-\mathrm{C}(2)-\mathrm{C}(1)$ & $118.4(2)$ & $\mathrm{C}(11)-\mathrm{C}(12)-\mathrm{C}(13)$ & $121.2(2)$ \\
\hline $\mathrm{O}(1)-\mathrm{C}(2)-\mathrm{C}(3)$ & $119.9(2)$ & $\mathrm{C}(14)-\mathrm{C}(13)-\mathrm{C}(12)$ & $119.0(2)$ \\
\hline $\mathrm{C}(1)-\mathrm{C}(2)-\mathrm{C}(3)$ & 121.6(3) & $\mathrm{C}(14)-\mathrm{C}(13)-\mathrm{C}(26)$ & $119.8(2)$ \\
\hline $\mathrm{C}(4)-\mathrm{C}(3)-\mathrm{C}(2)$ & 119.0(3) & $\mathrm{C}(12)-\mathrm{C}(13)-\mathrm{C}(26)$ & $121.1(2)$ \\
\hline$C(4)-C(3)-C(21)$ & $120.4(2)$ & $\mathrm{C}(13)-\mathrm{C}(14)-\mathrm{C}(15)$ & 122.2(3) \\
\hline $\mathrm{C}(2)-\mathrm{C}(3)-\mathrm{C}(21)$ & $120.6(2)$ & $\mathrm{C}(14)-\mathrm{C}(15)-\mathrm{C}(20)$ & $118.5(2)$ \\
\hline $\mathrm{C}(3)-\mathrm{C}(4)-\mathrm{C}(5)$ & 121.5(3) & $\mathrm{C}(14)-\mathrm{C}(15)-\mathrm{C}(16)$ & 122.6(3) \\
\hline $\mathrm{C}(4)-\mathrm{C}(5)-\mathrm{C}(10)$ & 119.0(3) & $\mathrm{C}(20)-\mathrm{C}(15)-\mathrm{C}(16)$ & 118.8(3) \\
\hline$C(4)-C(5)-C(6)$ & 121.6(3) & $\mathrm{C}(17)-\mathrm{C}(16)-\mathrm{C}(15)$ & 121.2(3) \\
\hline$C(10)-C(5)-C(6)$ & 119.3(3) & $\mathrm{C}(16)-\mathrm{C}(17)-\mathrm{C}(18)$ & 120.1(3) \\
\hline $\mathrm{C}(7)-\mathrm{C}(6)-\mathrm{C}(5)$ & $120.8(3)$ & $\mathrm{C}(19)-\mathrm{C}(18)-\mathrm{C}(17)$ & 120.2(3) \\
\hline $\mathrm{C}(6)-\mathrm{C}(7)-\mathrm{C}(8)$ & 119.9(3) & $\mathrm{C}(18)-\mathrm{C}(19)-\mathrm{C}(20)$ & 121.3(3) \\
\hline $\mathrm{C}(9)-\mathrm{C}(8)-\mathrm{C}(7)$ & 120.3(3) & $\mathrm{C}(11)-\mathrm{C}(20)-\mathrm{C}(15)$ & 119.6(3) \\
\hline C(8)-C(9)-C(10) & 121.6(3) & $\mathrm{C}(11)-\mathrm{C}(20)-\mathrm{C}(19)$ & 122.1(3) \\
\hline C(9)-C(10)-C(5) & 118.0(3) & $\mathrm{C}(15)-\mathrm{C}(20)-\mathrm{C}(19)$ & 118.2(2) \\
\hline $\mathrm{C}(9)-\mathrm{C}(10)-\mathrm{C}(1)$ & 122.7(3) & $\mathrm{C}(3)-\mathrm{C}(21)-\mathrm{P}(1)$ & $114.76(18)$ \\
\hline $\mathrm{C}(5)-\mathrm{C}(10)-\mathrm{C}(1)$ & 119.1(3) & $\mathrm{C}(23)-\mathrm{C}(22)-\mathrm{P}(1)$ & 112.5(3) \\
\hline$C(12)-C(11)-C(20)$ & $119.3(2)$ & $\mathrm{C}(25)-\mathrm{C}(24)-\mathrm{P}(1)$ & $112.0(3)$ \\
\hline$C(12)-C(11)-C(1)$ & $121.7(2)$ & $\mathrm{C}(13)-\mathrm{C}(26)-\mathrm{P}(2)$ & 112.31(19) \\
\hline
\end{tabular}




$\begin{array}{llll}\mathrm{C}(28)-\mathrm{C}(27)-\mathrm{P}(2) & 115.7(4) & \mathrm{C}(24)-\mathrm{P}(1)-\mathrm{C}(22) & 106.91(18) \\ \mathrm{C}(30)-\mathrm{C}(29)-\mathrm{P}(2) & 112.5(2) & \mathrm{O}(4)-\mathrm{P}(2)-\mathrm{C}(29) & 113.74(15) \\ \mathrm{O}(3)-\mathrm{P}(1)-\mathrm{C}(21) & 110.34(13) & \mathrm{O}(4)-\mathrm{P}(2)-\mathrm{C}(26) & 109.67(14) \\ \mathrm{O}(3)-\mathrm{P}(1)-\mathrm{C}(24) & 114.24(15) & \mathrm{C}(29)-\mathrm{P}(2)-\mathrm{C}(26) & 106.77(14) \\ \mathrm{C}(21)-\mathrm{P}(1)-\mathrm{C}(24) & 106.62(15) & \mathrm{O}(4)-\mathrm{P}(2)-\mathrm{C}(27) & 112.9(2) \\ \mathrm{O}(3)-\mathrm{P}(1)-\mathrm{C}(22) & 111.93(15) & \mathrm{C}(29)-\mathrm{P}(2)-\mathrm{C}(27) & 105.8(2) \\ \mathrm{C}(21)-\mathrm{P}(1)-\mathrm{C}(22) & 106.35(16) & \mathrm{C}(26)-\mathrm{P}(2)-\mathrm{C}(27) & 107.62(16)\end{array}$

Symmetry transformations used to generate equivalent atoms: 
Table S9. Anisotropic displacement parameters $\left(\AA^{2} \times 10^{3}\right)$ for $\mathbf{2}$. The anisotropic displacement factor exponent takes the form: $-2 \pi^{2}\left[\mathrm{~h}^{2} \mathrm{a}^{* 2} \mathrm{U}^{11}+\ldots+2 \mathrm{hka} * \mathrm{~b} * \mathrm{U}^{12}\right]$

\begin{tabular}{|c|c|c|c|c|c|c|}
\hline & $\mathrm{U}^{11}$ & $\mathrm{U}^{22}$ & $\mathrm{U}^{33}$ & $\mathrm{U}^{23}$ & $\mathrm{U}^{13}$ & $\mathrm{U}^{12}$ \\
\hline$\overline{C(1)}$ & $27(1)$ & $28(1)$ & $22(1)$ & $-3(1)$ & $-2(1)$ & $3(1)$ \\
\hline $\mathrm{C}(2)$ & $29(1)$ & $28(1)$ & $22(1)$ & $-3(1)$ & $2(1)$ & $3(1)$ \\
\hline C(3) & $33(2)$ & $28(1)$ & $24(1)$ & $-7(1)$ & $-1(1)$ & $4(1)$ \\
\hline C(4) & $30(2)$ & $33(2)$ & 33(1) & $-3(1)$ & $-8(1)$ & $6(1)$ \\
\hline$C(5)$ & $25(1)$ & $36(2)$ & $43(2)$ & $-8(1)$ & $-2(1)$ & $4(1)$ \\
\hline C(6) & $27(2)$ & $48(2)$ & 72(3) & $-3(2)$ & $-4(2)$ & $2(1)$ \\
\hline $\mathrm{C}(7)$ & $23(2)$ & $54(2)$ & 88(3) & $-10(2)$ & $8(2)$ & $-2(2)$ \\
\hline C(8) & $42(2)$ & $48(2)$ & $59(2)$ & $-9(2)$ & $19(2)$ & $-8(2)$ \\
\hline C(9) & $36(2)$ & $36(2)$ & $37(2)$ & $-7(1)$ & $8(1)$ & $-3(1)$ \\
\hline C(10) & $28(1)$ & $30(2)$ & $28(1)$ & $-7(1)$ & $3(1)$ & $-1(1)$ \\
\hline $\mathrm{C}(11)$ & $23(1)$ & $29(1)$ & $25(1)$ & $3(1)$ & $1(1)$ & $-1(1)$ \\
\hline C(12) & $28(1)$ & $31(2)$ & $21(1)$ & $-1(1)$ & $2(1)$ & $1(1)$ \\
\hline C(13) & $26(1)$ & $28(1)$ & $27(1)$ & $-2(1)$ & $1(1)$ & $-2(1)$ \\
\hline C(14) & 31(1) & $27(1)$ & $28(1)$ & $0(1)$ & $-1(1)$ & $1(1)$ \\
\hline$C(15)$ & $35(2)$ & $31(2)$ & $25(1)$ & $-5(1)$ & $-1(1)$ & $2(1)$ \\
\hline$C(16)$ & $57(2)$ & $49(2)$ & $26(1)$ & $-2(1)$ & $-3(1)$ & $13(2)$ \\
\hline $\mathrm{C}(17)$ & $79(3)$ & $60(2)$ & $25(2)$ & $-11(2)$ & $-2(2)$ & $24(2)$ \\
\hline C(18) & $64(2)$ & $59(2)$ & $36(2)$ & $-14(2)$ & $-9(2)$ & $30(2)$ \\
\hline C(19) & $41(2)$ & $41(2)$ & $30(2)$ & $-6(1)$ & $-4(1)$ & $13(1)$ \\
\hline C(20) & $29(1)$ & $31(2)$ & $24(1)$ & $-3(1)$ & $-2(1)$ & $0(1)$ \\
\hline $\mathrm{C}(21)$ & $39(2)$ & $30(2)$ & $20(1)$ & $-2(1)$ & $0(1)$ & $4(1)$ \\
\hline$C(22)$ & $60(2)$ & $46(2)$ & $37(2)$ & $12(1)$ & $8(2)$ & $-5(2)$ \\
\hline C(23) & 71(3) & 68(3) & $56(2)$ & $-4(2)$ & $35(2)$ & $-13(2)$ \\
\hline$C(24)$ & $51(2)$ & $42(2)$ & $53(2)$ & $-13(2)$ & $10(2)$ & $1(2)$ \\
\hline$C(25)$ & $75(3)$ & $71(3)$ & $112(4)$ & $-50(3)$ & $37(3)$ & $-15(2)$ \\
\hline$C(26)$ & $28(1)$ & $30(2)$ & $26(1)$ & $-2(1)$ & $-1(1)$ & $1(1)$ \\
\hline C(27) & $76(3)$ & $56(3)$ & $114(4)$ & $-37(3)$ & $56(3)$ & $-35(2)$ \\
\hline C(28) & $110(5)$ & $77(4)$ & $165(6)$ & $-46(4)$ & $89(5)$ & $-30(3)$ \\
\hline C(29) & $44(2)$ & $37(2)$ & $59(2)$ & $-16(2)$ & $-3(2)$ & $12(2)$ \\
\hline C(30) & $61(2)$ & $32(2)$ & $61(2)$ & $-7(2)$ & $3(2)$ & $0(2)$ \\
\hline $\mathrm{O}(1)$ & $27(1)$ & $34(1)$ & 38(1) & $7(1)$ & $2(1)$ & $0(1)$ \\
\hline $\mathrm{O}(2)$ & $48(1)$ & $43(1)$ & $22(1)$ & $-5(1)$ & $1(1)$ & $14(1)$ \\
\hline $\mathrm{O}(3)$ & $42(1)$ & $43(1)$ & 28(1) & $0(1)$ & $-3(1)$ & $-8(1)$ \\
\hline $\mathrm{O}(4)$ & $46(1)$ & $44(1)$ & $51(2)$ & $-16(1)$ & $-17(1)$ & $7(1)$ \\
\hline$P(1)$ & $40(1)$ & $31(1)$ & $24(1)$ & $-2(1)$ & $4(1)$ & $-2(1)$ \\
\hline $\mathrm{P}(2)$ & $31(1)$ & $32(1)$ & $39(1)$ & $-11(1)$ & $-1(1)$ & $1(1)$ \\
\hline
\end{tabular}


Table S10. Hydrogen coordinates $\left(\times 10^{4}\right)$ and isotropic displacement parameters $\left(\AA^{2} \times 10^{3}\right)$ for 2.

\begin{tabular}{|c|c|c|c|c|}
\hline & $\mathrm{x}$ & $\mathrm{y}$ & $\mathrm{z}$ & $\mathrm{U}(\mathrm{eq})$ \\
\hline $\mathrm{H}(4)$ & -1838 & 4568 & 5081 & 42 \\
\hline $\mathrm{H}(6)$ & -4142 & 3882 & 5591 & 63 \\
\hline $\mathrm{H}(7)$ & -5133 & 2976 & 6898 & 67 \\
\hline $\mathrm{H}(8)$ & -3395 & 2224 & 8676 & 58 \\
\hline $\mathrm{H}(9)$ & -705 & 2338 & 9017 & 44 \\
\hline $\mathrm{H}(14)$ & 4650 & 1650 & 11839 & 36 \\
\hline $\mathrm{H}(16)$ & 4056 & 2495 & 13760 & 56 \\
\hline $\mathrm{H}(17)$ & 2726 & 3522 & 14392 & 69 \\
\hline $\mathrm{H}(18)$ & 1153 & 4324 & 12583 & 68 \\
\hline $\mathrm{H}(19)$ & 894 & 4057 & 10176 & 48 \\
\hline $\mathrm{H}(21 \mathrm{~A})$ & 232 & 5137 & 4363 & 37 \\
\hline $\mathrm{H}(21 \mathrm{~B})$ & 1922 & 4731 & 4828 & 37 \\
\hline $\mathrm{H}(22 \mathrm{~A})$ & 1896 & 6500 & 4083 & 58 \\
\hline $\mathrm{H}(22 \mathrm{~B})$ & 3031 & 6924 & 5442 & 58 \\
\hline $\mathrm{H}(23 \mathrm{~A})$ & 4914 & 5891 & 5540 & 92 \\
\hline $\mathrm{H}(23 \mathrm{~B})$ & 4632 & 6394 & 4089 & 92 \\
\hline $\mathrm{H}(23 \mathrm{C})$ & 3811 & 5564 & 4062 & 92 \\
\hline $\mathrm{H}(24 \mathrm{~A})$ & -391 & 6570 & 5597 & 60 \\
\hline $\mathrm{H}(24 \mathrm{~B})$ & -417 & 6013 & 6929 & 60 \\
\hline $\mathrm{H}(25 \mathrm{~A})$ & 1514 & 6843 & 8453 & 125 \\
\hline $\mathrm{H}(25 \mathrm{~B})$ & -136 & 7269 & 7810 & 125 \\
\hline $\mathrm{H}(25 \mathrm{C})$ & 1319 & 7424 & 7114 & 125 \\
\hline $\mathrm{H}(26 \mathrm{~A})$ & 5349 & 856 & 10161 & 35 \\
\hline $\mathrm{H}(26 \mathrm{~B})$ & 5016 & 1146 & 8521 & 35 \\
\hline $\mathrm{H}(27 \mathrm{~A})$ & 1858 & -487 & 10205 & 92 \\
\hline H(27B) & 2930 & 165 & 11156 & 92 \\
\hline $\mathrm{H}(28 \mathrm{~A})$ & 1024 & 1081 & 10395 & 162 \\
\hline $\mathrm{H}(28 \mathrm{~B})$ & 192 & 345 & 10903 & 162 \\
\hline $\mathrm{H}(28 \mathrm{C})$ & -5 & 505 & 9216 & 162 \\
\hline $\mathrm{H}(29 \mathrm{~A})$ & 4891 & -513 & 7954 & 59 \\
\hline $\mathrm{H}(29 \mathrm{~B})$ & 4965 & -703 & 9610 & 59 \\
\hline $\mathrm{H}(30 \mathrm{~A})$ & 2558 & -1412 & 8864 & 81 \\
\hline $\mathrm{H}(30 \mathrm{~B})$ & 3872 & -1770 & 8153 & 81 \\
\hline $\mathrm{H}(30 \mathrm{C})$ & 2510 & -1231 & 7210 & 81 \\
\hline $\mathrm{H}(1)$ & 3230 & 4546 & 7499 & 51 \\
\hline $\mathrm{H}(2)$ & 2220 & 1521 & 7103 & 59 \\
\hline
\end{tabular}


Table S11. Crystal data and structure refinement for 3.

Identification code

Empirical formula

Formula weight

Temperature

Wavelength

Crystal system

Space group

Unit cell dimensions

Volume

$\mathrm{Z}$

Density (calculated)

Absorption coefficient

$\mathrm{F}(000)$

Crystal size

Theta range for data collection

Index ranges

Reflections collected

Independent reflections

Completeness to theta $=28.73^{\circ}$

Absorption correction

Max. and min. transmission

Refinement method

Data / restraints / parameters

Goodness-of-fit on $\mathrm{F}^{2}$

Final R indices [I $>2 \operatorname{sigma(I)]~}$

$\mathrm{R}$ indices (all data)

Absolute structure parameter

Largest diff. peak and hole
3 (s76p1 ,BINOL-CH${ }_{2} \mathrm{POBu}_{2}^{\mathrm{t}}$.toluene)

C45 H60 O4 P2

726.87

153(2) K

$0.71073 \AA$

Triclinic

P1

$\mathrm{a}=11.5165(15) \AA \quad \alpha=108.081(2)^{\circ}$

$\mathrm{b}=11.7891(15) \AA \quad \beta=101.365(2)^{\circ}$

$\mathrm{c}=16.411(2) \AA \quad \gamma=90.766(2)^{\circ}$

2069.9(5) $\AA^{3}$

2

$1.166 \mathrm{~g} / \mathrm{cm}^{3}$

$0.145 \mathrm{~mm}^{-1}$

784

$0.35 \times 0.33 \times 0.29 \mathrm{~mm}^{3}$

1.34 to $28.73^{\circ}$

$-14 \leq \mathrm{h} \leq 15,-15 \leq \mathrm{k} \leq 15,-22 \leq \mathrm{l} \leq 21$

19142

$16574[\mathrm{R}(\mathrm{int})=0.0192]$

$89.9 \%$

Semi-empirical from equivalents

0.9715 and 0.9577

Full-matrix least-squares on $\mathrm{F}^{2}$

16574 / 3 / 949

1.013

$\mathrm{R} 1=0.0535, \mathrm{wR} 2=0.1370$

$\mathrm{R} 1=0.0695, \mathrm{wR} 2=0.1514$

$-0.05(6)$

0.547 and -0.316 e. $\AA^{-3}$ 
Table S12. Atomic coordinates $\left(\times 10^{4}\right)$ and equivalent isotropic displacement parameters $\left(\AA^{2} \times\right.$ $10^{3}$ ) for 3 . $U(e q)$ is defined as one third of the trace of the orthogonalized $U^{i j}$ tensor.

\begin{tabular}{|c|c|c|c|c|}
\hline & $x$ & $\mathrm{y}$ & $\mathrm{z}$ & $\mathrm{U}(\mathrm{eq})$ \\
\hline $\mathrm{C}(1)$ & $8311(2)$ & $7225(2)$ & $7705(2)$ & $30(1)$ \\
\hline $\mathrm{C}(2)$ & 7263(3) & $7395(2)$ & $8000(2)$ & 31(1) \\
\hline C(3) & $6779(3)$ & 6530(3) & $8320(2)$ & 31(1) \\
\hline C(4) & 7382(3) & 5539(3) & $8341(2)$ & $36(1)$ \\
\hline C(5) & $8457(3)$ & 5340(3) & $8059(2)$ & $36(1)$ \\
\hline C(6) & $9079(3)$ & 4318(3) & $8079(2)$ & $45(1)$ \\
\hline C(7) & 10132(3) & 4149(3) & $7811(2)$ & $48(1)$ \\
\hline C(8) & 10618(3) & 5009(3) & $7504(2)$ & $44(1)$ \\
\hline C(9) & 10033(3) & 5988(3) & $7456(2)$ & $37(1)$ \\
\hline$C(10)$ & 8937(3) & 6194(3) & $7737(2)$ & $32(1)$ \\
\hline $\mathrm{C}(11)$ & $8804(2)$ & 8131(2) & $7378(2)$ & $30(1)$ \\
\hline $\mathrm{C}(12)$ & 8635(3) & 7962(3) & $6498(2)$ & $33(1)$ \\
\hline C(13) & 9106(3) & 8824(3) & $6168(2)$ & $34(1)$ \\
\hline $\mathrm{C}(14)$ & 9777(3) & 9815(3) & $6751(2)$ & $36(1)$ \\
\hline$C(15)$ & 10025(3) & 10007(3) & 7663(2) & $36(1)$ \\
\hline$C(16)$ & 10775(3) & 10999(3) & $8269(2)$ & $45(1)$ \\
\hline $\mathrm{C}(17)$ & 10987(3) & 11174(3) & $9147(3)$ & 53(1) \\
\hline C(18) & 10466(3) & 10359(3) & $9468(2)$ & $48(1)$ \\
\hline C(19) & $9754(3)$ & 9383(3) & $8912(2)$ & $40(1)$ \\
\hline $\mathrm{C}(20)$ & $9512(3)$ & 9167(3) & $7986(2)$ & $32(1)$ \\
\hline $\mathrm{C}(21)$ & $5545(3)$ & 6591(3) & $8516(2)$ & $33(1)$ \\
\hline $\mathrm{C}(22)$ & 5938(3) & 7894(3) & 10447(2) & $36(1)$ \\
\hline C(23) & $7263(4)$ & $7826(5)$ & 10442(3) & $70(1)$ \\
\hline$C(24)$ & $5512(4)$ & 6825(3) & 10690(3) & 61(1) \\
\hline C(25) & $5803(4)$ & 9063(3) & $11142(2)$ & $56(1)$ \\
\hline C(26) & 3537(3) & 7708(3) & $9149(2)$ & $44(1)$ \\
\hline C(27) & 3085(3) & $6418(4)$ & $9035(3)$ & 61(1) \\
\hline C(28) & $3075(4)$ & 8566(4) & 9905(3) & $63(1)$ \\
\hline C(29) & 3061(3) & $8005(4)$ & 8305(3) & $63(1)$ \\
\hline C(30) & 8781(3) & $8687(2)$ & $5207(2)$ & $33(1)$ \\
\hline $\mathrm{C}(31)$ & 10870(3) & 7856(3) & $4421(2)$ & 41(1) \\
\hline C(32) & 11442(3) & 6744(3) & 3943(3) & $47(1)$ \\
\hline C(33) & $10928(4)$ & 8809(3) & 3956(3) & 54(1) \\
\hline C(34) & 11573(3) & 8370(3) & 5368(3) & 51(1) \\
\hline C(35) & 8275(3) & 7148(3) & $3402(2)$ & $44(1)$ \\
\hline C(36) & $7911(4)$ & 8315(3) & $3224(3)$ & $57(1)$ \\
\hline C(37) & $8741(4)$ & 6342(3) & 2622(2) & $55(1)$ \\
\hline C(38) & 7165(3) & 6473(3) & 3474(3) & $52(1)$ \\
\hline C(39) & $3707(2)$ & $2076(2)$ & $6507(2)$ & $32(1)$ \\
\hline$C(40)$ & 3964(3) & 2238(3) & $7387(2)$ & $33(1)$ \\
\hline
\end{tabular}




\begin{tabular}{|c|c|c|c|c|}
\hline$C(41)$ & 4587(3) & 1391(3) & $7734(2)$ & $35(1)$ \\
\hline $\mathrm{C}(42)$ & 4952(3) & 418(3) & $7158(2)$ & $39(1)$ \\
\hline$C(43)$ & $4750(3)$ & 236(3) & $6254(2)$ & $39(1)$ \\
\hline$C(44)$ & 5172(3) & $-748(3)$ & 5665(3) & 49(1) \\
\hline$C(45)$ & 4949(4) & $-923(3)$ & 4792(3) & $57(1)$ \\
\hline $\mathrm{C}(46)$ & 4291(3) & $-99(3)$ & 4456(3) & $49(1)$ \\
\hline $\mathrm{C}(47)$ & 3892(3) & 863(3) & 4999(2) & $40(1)$ \\
\hline C(48) & 4102(3) & 1070(3) & $5911(2)$ & $34(1)$ \\
\hline C(49) & 3063(3) & 2992(3) & $6172(2)$ & 31(1) \\
\hline$C(50)$ & 1843(3) & $2844(2)$ & $5896(2)$ & $31(1)$ \\
\hline$C(51)$ & $1210(2)$ & $3710(2)$ & $5568(2)$ & 31(1) \\
\hline$C(52)$ & 1839(3) & 4698(3) & $5560(2)$ & $36(1)$ \\
\hline C(53) & 3091(3) & 4866(3) & $5823(2)$ & $36(1)$ \\
\hline$C(54)$ & 3741(3) & $5874(3)$ & $5789(2)$ & $45(1)$ \\
\hline C(55) & 4949(3) & 5999(3) & $6017(2)$ & $46(1)$ \\
\hline$C(56)$ & 5573(3) & 5125(3) & $6296(2)$ & $42(1)$ \\
\hline$C(57)$ & 4972(3) & 4162(3) & $6366(2)$ & $36(1)$ \\
\hline $\mathrm{C}(58)$ & 3709(3) & 3999(3) & $6128(2)$ & $32(1)$ \\
\hline C(59) & 4743(3) & 1525(3) & $8699(2)$ & $38(1)$ \\
\hline $\mathrm{C}(60)$ & 7245(3) & 2412(3) & $9617(2)$ & $38(1)$ \\
\hline$C(61)$ & 7478(3) & 1852(3) & $8700(2)$ & $45(1)$ \\
\hline$C(62)$ & 7529(3) & 1531(3) & $10135(2)$ & $46(1)$ \\
\hline C(63) & 8062(3) & $3580(3)$ & 10079(2) & $46(1)$ \\
\hline C(64) & 5101(3) & 3063(3) & 10496(2) & $43(1)$ \\
\hline C(65) & $3935(4)$ & $3649(3)$ & 10342(3) & $54(1)$ \\
\hline C(66) & $5926(4)$ & 3949(3) & 11299(2) & $55(1)$ \\
\hline $\mathrm{C}(67)$ & $4834(4)$ & 1897(3) & 10687(3) & $59(1)$ \\
\hline C(68) & $-126(3)$ & $3620(3)$ & $5345(2)$ & $34(1)$ \\
\hline C(69) & $-592(3)$ & 2293(3) & $3431(2)$ & $44(1)$ \\
\hline $\mathrm{C}(70)$ & $-820(7)$ & $3445(4)$ & $3223(4)$ & $100(2)$ \\
\hline $\mathrm{C}(71)$ & $-1263(6)$ & 1257(5) & 2671(3) & $95(2)$ \\
\hline $\mathrm{C}(72)$ & 715(5) & 2066(6) & 3515(3) & $94(2)$ \\
\hline C(73) & $-2501(3)$ & 2501(3) & 4564(3) & $52(1)$ \\
\hline $\mathrm{C}(74)$ & $-3301(4)$ & $1473(4)$ & $3843(5)$ & 109(3) \\
\hline$C(75)$ & $-2931(4)$ & $3691(4)$ & $4505(4)$ & 70(1) \\
\hline $\mathrm{C}(76)$ & $-2591(4)$ & 2397(7) & $5462(5)$ & $102(2)$ \\
\hline $\mathrm{C}(77)$ & $2270(5)$ & 7164(8) & $1664(4)$ & $97(2)$ \\
\hline$C(78)$ & 1760(9) & $6247(6)$ & $971(5)$ & $110(2)$ \\
\hline C(79) & 888(8) & $6082(14)$ & $364(7)$ & 168(5) \\
\hline $\mathrm{C}(80)$ & 292(7) & $7146(9)$ & 481(6) & $111(2)$ \\
\hline C(81) & 594(7) & 8175(8) & $1103(6)$ & $101(2)$ \\
\hline C(82) & 1605(8) & $8312(7)$ & $1776(6)$ & 121(3) \\
\hline C(83) & 3308(11) & 7319(13) & 2326(6) & 236(8) \\
\hline $\mathrm{C}(84)$ & $3351(6)$ & $3624(7)$ & $2950(5)$ & $111(2)$ \\
\hline $\mathrm{C}(85)$ & 2376(6) & $3560(9)$ & 2237(6) & $122(3)$ \\
\hline
\end{tabular}




\begin{tabular}{llllr}
$\mathrm{C}(86)$ & $1882(9)$ & $2516(10)$ & $1629(5)$ & $135(4)$ \\
$\mathrm{C}(87)$ & $2380(7)$ & $1560(10)$ & $1772(5)$ & $122(3)$ \\
$\mathrm{C}(88)$ & $3300(6)$ & $1548(8)$ & $2423(4)$ & $113(2)$ \\
$\mathrm{C}(89)$ & $3743(6)$ & $2522(8)$ & $3012(5)$ & $106(2)$ \\
$\mathrm{C}(90)$ & $3979(9)$ & $4753(11)$ & $3503(6)$ & $213(7)$ \\
$\mathrm{O}(1)$ & $6688(2)$ & $8368(2)$ & $7931(2)$ & $37(1)$ \\
$\mathrm{O}(2)$ & $7965(2)$ & $6958(2)$ & $5929(1)$ & $40(1)$ \\
$\mathrm{O}(3)$ & $5562(2)$ & $9042(2)$ & $9216(1)$ & $36(1)$ \\
$\mathrm{O}(4)$ & $9323(2)$ & $6319(2)$ & $4745(2)$ & $39(1)$ \\
$\mathrm{O}(5)$ & $3587(2)$ & $3227(2)$ & $7942(2)$ & $40(1)$ \\
$\mathrm{O}(6)$ & $1264(2)$ & $1892(2)$ & $5980(2)$ & $39(1)$ \\
$\mathrm{O}(7)$ & $5555(2)$ & $3892(2)$ & $9189(2)$ & $40(1)$ \\
$\mathrm{O}(8)$ & $-585(2)$ & $1179(2)$ & $4665(2)$ & $38(1)$ \\
$\mathrm{P}(1)$ & $5173(1)$ & $7895(1)$ & $9343(1)$ & $32(1)$ \\
$\mathrm{P}(2)$ & $9341(1)$ & $7421(1)$ & $4457(1)$ & $33(1)$ \\
$\mathrm{P}(3)$ & $5684(1)$ & $2802(1)$ & $9488(1)$ & $33(1)$ \\
$\mathrm{P}(4)$ & $-938(1)$ & $2321(1)$ & $4486(1)$ & $33(1)$ \\
\hline
\end{tabular}


Table S13. Bond lengths $[\AA]$ and angles $\left[{ }^{\circ}\right]$ for $\mathbf{3}$.

\begin{tabular}{|c|c|c|c|}
\hline $\mathrm{C}(1)-\mathrm{C}(2)$ & $1.380(4)$ & $\mathrm{C}(35)-\mathrm{C}(38)$ & $1.540(5)$ \\
\hline $\mathrm{C}(1)-\mathrm{C}(10)$ & $1.431(4)$ & $\mathrm{C}(35)-\mathrm{P}(2)$ & 1.848(3) \\
\hline $\mathrm{C}(1)-\mathrm{C}(11)$ & $1.487(4)$ & C(39)-C(40) & $1.366(4)$ \\
\hline $\mathrm{C}(2)-\mathrm{O}(1)$ & 1.357(3) & C(39)-C(48) & $1.428(4)$ \\
\hline $\mathrm{C}(2)-\mathrm{C}(3)$ & $1.435(4)$ & C(39)-C(49) & $1.499(4)$ \\
\hline$C(3)-C(4)$ & $1.374(4)$ & $\mathrm{C}(40)-\mathrm{O}(5)$ & $1.374(4)$ \\
\hline$C(3)-C(21)$ & $1.515(4)$ & $\mathrm{C}(40)-\mathrm{C}(41)$ & $1.432(4)$ \\
\hline$C(4)-C(5)$ & $1.404(4)$ & $\mathrm{C}(41)-\mathrm{C}(42)$ & $1.372(5)$ \\
\hline$C(5)-C(6)$ & $1.416(4)$ & $\mathrm{C}(41)-\mathrm{C}(59)$ & $1.515(4)$ \\
\hline$C(5)-C(10)$ & $1.423(4)$ & $C(42)-C(43)$ & $1.403(5)$ \\
\hline$C(6)-C(7)$ & $1.366(5)$ & $\mathrm{C}(43)-\mathrm{C}(44)$ & $1.421(5)$ \\
\hline$C(7)-C(8)$ & $1.416(5)$ & $\mathrm{C}(43)-\mathrm{C}(48)$ & $1.428(4)$ \\
\hline $\mathrm{C}(8)-\mathrm{C}(9)$ & $1.360(4)$ & $\mathrm{C}(44)-\mathrm{C}(45)$ & $1.354(6)$ \\
\hline $\mathrm{C}(9)-\mathrm{C}(10)$ & $1.426(4)$ & $\mathrm{C}(45)-\mathrm{C}(46)$ & $1.417(5)$ \\
\hline $\mathrm{C}(11)-\mathrm{C}(12)$ & $1.368(4)$ & $\mathrm{C}(46)-\mathrm{C}(47)$ & $1.357(5)$ \\
\hline$C(11)-C(20)$ & $1.432(4)$ & $C(47)-C(48)$ & $1.410(5)$ \\
\hline $\mathrm{C}(12)-\mathrm{O}(2)$ & $1.369(3)$ & $\mathrm{C}(49)-\mathrm{C}(50)$ & $1.380(4)$ \\
\hline $\mathrm{C}(12)-\mathrm{C}(13)$ & $1.436(4)$ & C(49)-C(58) & $1.422(4)$ \\
\hline $\mathrm{C}(13)-\mathrm{C}(14)$ & $1.367(4)$ & $\mathrm{C}(50)-\mathrm{O}(6)$ & $1.355(3)$ \\
\hline $\mathrm{C}(13)-\mathrm{C}(30)$ & $1.504(4)$ & $\mathrm{C}(50)-\mathrm{C}(51)$ & $1.438(4)$ \\
\hline $\mathrm{C}(14)-\mathrm{C}(15)$ & $1.411(5)$ & $\mathrm{C}(51)-\mathrm{C}(52)$ & $1.368(4)$ \\
\hline $\mathrm{C}(15)-\mathrm{C}(20)$ & $1.429(4)$ & $\mathrm{C}(51)-\mathrm{C}(68)$ & $1.504(4)$ \\
\hline $\mathrm{C}(15)-\mathrm{C}(16)$ & $1.420(4)$ & $\mathrm{C}(52)-\mathrm{C}(53)$ & $1.414(4)$ \\
\hline $\mathrm{C}(16)-\mathrm{C}(17)$ & $1.363(5)$ & $\mathrm{C}(53)-\mathrm{C}(58)$ & $1.413(4)$ \\
\hline $\mathrm{C}(17)-\mathrm{C}(18)$ & $1.407(5)$ & $\mathrm{C}(53)-\mathrm{C}(54)$ & $1.418(4)$ \\
\hline $\mathrm{C}(18)-\mathrm{C}(19)$ & $1.363(5)$ & $\mathrm{C}(54)-\mathrm{C}(55)$ & $1.361(5)$ \\
\hline $\mathrm{C}(19)-\mathrm{C}(20)$ & $1.429(4)$ & $\mathrm{C}(55)-\mathrm{C}(56)$ & $1.403(5)$ \\
\hline $\mathrm{C}(21)-\mathrm{P}(1)$ & 1.828(3) & $\mathrm{C}(56)-\mathrm{C}(57)$ & $1.367(4)$ \\
\hline $\mathrm{C}(22)-\mathrm{C}(25)$ & $1.524(5)$ & $\mathrm{C}(57)-\mathrm{C}(58)$ & $1.424(4)$ \\
\hline $\mathrm{C}(22)-\mathrm{C}(24)$ & $1.538(5)$ & C(59)-P(3) & $1.819(3)$ \\
\hline $\mathrm{C}(22)-\mathrm{C}(23)$ & $1.531(5)$ & $\mathrm{C}(60)-\mathrm{C}(61)$ & $1.523(5)$ \\
\hline $\mathrm{C}(22)-\mathrm{P}(1)$ & 1.852(3) & $\mathrm{C}(60)-\mathrm{C}(62)$ & $1.534(4)$ \\
\hline $\mathrm{C}(26)-\mathrm{C}(28)$ & $1.529(5)$ & $\mathrm{C}(60)-\mathrm{C}(63)$ & $1.547(4)$ \\
\hline $\mathrm{C}(26)-\mathrm{C}(29)$ & $1.532(5)$ & $\mathrm{C}(60)-\mathrm{P}(3)$ & $1.849(3)$ \\
\hline $\mathrm{C}(26)-\mathrm{C}(27)$ & $1.544(5)$ & $\mathrm{C}(64)-\mathrm{C}(66)$ & $1.535(5)$ \\
\hline $\mathrm{C}(26)-\mathrm{P}(1)$ & $1.847(3)$ & $\mathrm{C}(64)-\mathrm{C}(65)$ & $1.532(5)$ \\
\hline $\mathrm{C}(30)-\mathrm{P}(2)$ & 1.832(3) & $\mathrm{C}(64)-\mathrm{C}(67)$ & $1.541(4)$ \\
\hline $\mathrm{C}(31)-\mathrm{C}(33)$ & $1.549(5)$ & $\mathrm{C}(64)-\mathrm{P}(3)$ & $1.847(3)$ \\
\hline $\mathrm{C}(31)-\mathrm{C}(34)$ & $1.532(5)$ & $\mathrm{C}(68)-\mathrm{P}(4)$ & $1.819(3)$ \\
\hline $\mathrm{C}(31)-\mathrm{C}(32)$ & $1.534(5)$ & $\mathrm{C}(69)-\mathrm{C}(70)$ & $1.514(5)$ \\
\hline $\mathrm{C}(31)-\mathrm{P}(2)$ & $1.844(3)$ & $\mathrm{C}(69)-\mathrm{C}(71)$ & $1.514(6)$ \\
\hline $\mathrm{C}(35)-\mathrm{C}(37)$ & $1.536(5)$ & $\mathrm{C}(69)-\mathrm{C}(72)$ & $1.520(6)$ \\
\hline$C(35)-C(36)$ & $1.540(4)$ & $\mathrm{C}(69)-\mathrm{P}(4)$ & $1.843(4)$ \\
\hline
\end{tabular}




\begin{tabular}{|c|c|c|c|}
\hline $\mathrm{C}(73)-\mathrm{C}(76)$ & $1.540(7)$ & $\mathrm{C}(84)-\mathrm{C}(89)$ & $1.409(10)$ \\
\hline $\mathrm{C}(73)-\mathrm{C}(75)$ & $1.519(5)$ & $\mathrm{C}(84)-\mathrm{C}(85)$ & $1.436(10)$ \\
\hline $\mathrm{C}(73)-\mathrm{C}(74)$ & $1.539(6)$ & $\mathrm{C}(84)-\mathrm{C}(90)$ & $1.443(13)$ \\
\hline $\mathrm{C}(73)-\mathrm{P}(4)$ & $1.840(3)$ & $\mathrm{C}(85)-\mathrm{C}(86)$ & $1.353(11)$ \\
\hline $\mathrm{C}(77)-\mathrm{C}(78)$ & $1.329(9)$ & $\mathrm{C}(86)-\mathrm{C}(87)$ & $1.336(12)$ \\
\hline $\mathrm{C}(77)-\mathrm{C}(83)$ & $1.415(13)$ & $\mathrm{C}(87)-\mathrm{C}(88)$ & $1.352(10)$ \\
\hline $\mathrm{C}(77)-\mathrm{C}(82)$ & $1.542(10)$ & $\mathrm{C}(88)-\mathrm{C}(89)$ & $1.271(10)$ \\
\hline $\mathrm{C}(78)-\mathrm{C}(79)$ & $1.231(11)$ & $\mathrm{O}(3)-\mathrm{P}(1)$ & $1.506(2)$ \\
\hline $\mathrm{C}(79)-\mathrm{C}(80)$ & $1.417(13)$ & $\mathrm{O}(4)-\mathrm{P}(2)$ & $1.515(2)$ \\
\hline $\mathrm{C}(80)-\mathrm{C}(81)$ & $1.309(10)$ & $\mathrm{O}(7)-\mathrm{P}(3)$ & $1.510(2)$ \\
\hline $\mathrm{C}(81)-\mathrm{C}(82)$ & $1.406(11)$ & $\mathrm{O}(8)-\mathrm{P}(4)$ & $1.508(2)$ \\
\hline$C(2)-C(1)-C(10)$ & $120.0(2)$ & $\mathrm{C}(17)-\mathrm{C}(16)-\mathrm{C}(15)$ & $121.0(3)$ \\
\hline $\mathrm{C}(2)-\mathrm{C}(1)-\mathrm{C}(11)$ & $119.9(2)$ & $\mathrm{C}(16)-\mathrm{C}(17)-\mathrm{C}(18)$ & $120.0(3)$ \\
\hline $\mathrm{C}(10)-\mathrm{C}(1)-\mathrm{C}(11)$ & 120.1(3) & $\mathrm{C}(19)-\mathrm{C}(18)-\mathrm{C}(17)$ & 121.1(3) \\
\hline $\mathrm{O}(1)-\mathrm{C}(2)-\mathrm{C}(1)$ & $117.6(2)$ & $\mathrm{C}(18)-\mathrm{C}(19)-\mathrm{C}(20)$ & $120.5(3)$ \\
\hline $\mathrm{O}(1)-\mathrm{C}(2)-\mathrm{C}(3)$ & 121.8(3) & $\mathrm{C}(15)-\mathrm{C}(20)-\mathrm{C}(11)$ & 119.4(3) \\
\hline $\mathrm{C}(1)-\mathrm{C}(2)-\mathrm{C}(3)$ & $120.5(3)$ & $\mathrm{C}(15)-\mathrm{C}(20)-\mathrm{C}(19)$ & 118.3(3) \\
\hline $\mathrm{C}(4)-\mathrm{C}(3)-\mathrm{C}(2)$ & 119.0(3) & $\mathrm{C}(11)-\mathrm{C}(20)-\mathrm{C}(19)$ & 122.3(3) \\
\hline $\mathrm{C}(4)-\mathrm{C}(3)-\mathrm{C}(21)$ & 118.7(3) & $\mathrm{C}(3)-\mathrm{C}(21)-\mathrm{P}(1)$ & $120.5(2)$ \\
\hline $\mathrm{C}(2)-\mathrm{C}(3)-\mathrm{C}(21)$ & 121.7(3) & $C(25)-C(22)-C(24)$ & $110.0(3)$ \\
\hline $\mathrm{C}(3)-\mathrm{C}(4)-\mathrm{C}(5)$ & 122.2(3) & $C(25)-C(22)-C(23)$ & $107.4(3)$ \\
\hline$C(6)-C(5)-C(4)$ & 122.3(3) & $C(24)-C(22)-C(23)$ & $108.4(3)$ \\
\hline$C(6)-C(5)-C(10)$ & 118.9(3) & $\mathrm{C}(25)-\mathrm{C}(22)-\mathrm{P}(1)$ & $110.6(2)$ \\
\hline $\mathrm{C}(4)-\mathrm{C}(5)-\mathrm{C}(10)$ & 118.9(3) & $\mathrm{C}(24)-\mathrm{C}(22)-\mathrm{P}(1)$ & $113.2(2)$ \\
\hline $\mathrm{C}(7)-\mathrm{C}(6)-\mathrm{C}(5)$ & 121.3(3) & $\mathrm{C}(23)-\mathrm{C}(22)-\mathrm{P}(1)$ & $107.0(2)$ \\
\hline $\mathrm{C}(6)-\mathrm{C}(7)-\mathrm{C}(8)$ & 119.7(3) & $\mathrm{C}(28)-\mathrm{C}(26)-\mathrm{C}(29)$ & 109.3(3) \\
\hline $\mathrm{C}(9)-\mathrm{C}(8)-\mathrm{C}(7)$ & $120.6(3)$ & $\mathrm{C}(28)-\mathrm{C}(26)-\mathrm{C}(27)$ & $108.4(3)$ \\
\hline $\mathrm{C}(8)-\mathrm{C}(9)-\mathrm{C}(10)$ & $121.0(3)$ & $C(29)-C(26)-C(27)$ & $109.5(3)$ \\
\hline $\mathrm{C}(1)-\mathrm{C}(10)-\mathrm{C}(5)$ & 119.4(3) & $\mathrm{C}(28)-\mathrm{C}(26)-\mathrm{P}(1)$ & 111.2(3) \\
\hline $\mathrm{C}(1)-\mathrm{C}(10)-\mathrm{C}(9)$ & 122.2(3) & $\mathrm{C}(29)-\mathrm{C}(26)-\mathrm{P}(1)$ & $106.3(2)$ \\
\hline $\mathrm{C}(5)-\mathrm{C}(10)-\mathrm{C}(9)$ & 118.4(3) & $\mathrm{C}(27)-\mathrm{C}(26)-\mathrm{P}(1)$ & $112.0(2)$ \\
\hline $\mathrm{C}(12)-\mathrm{C}(11)-\mathrm{C}(20)$ & $119.3(2)$ & $\mathrm{C}(13)-\mathrm{C}(30)-\mathrm{P}(2)$ & $117.0(2)$ \\
\hline $\mathrm{C}(12)-\mathrm{C}(11)-\mathrm{C}(1)$ & $120.8(2)$ & $\mathrm{C}(33)-\mathrm{C}(31)-\mathrm{C}(34)$ & 108.7(3) \\
\hline $\mathrm{C}(20)-\mathrm{C}(11)-\mathrm{C}(1)$ & 119.8(3) & $\mathrm{C}(33)-\mathrm{C}(31)-\mathrm{C}(32)$ & 108.8(3) \\
\hline $\mathrm{O}(2)-\mathrm{C}(12)-\mathrm{C}(11)$ & $118.2(2)$ & $\mathrm{C}(34)-\mathrm{C}(31)-\mathrm{C}(32)$ & 109.1(3) \\
\hline $\mathrm{O}(2)-\mathrm{C}(12)-\mathrm{C}(13)$ & $120.0(3)$ & $\mathrm{C}(33)-\mathrm{C}(31)-\mathrm{P}(2)$ & $113.1(2)$ \\
\hline $\mathrm{C}(11)-\mathrm{C}(12)-\mathrm{C}(13)$ & 121.7(3) & $\mathrm{C}(34)-\mathrm{C}(31)-\mathrm{P}(2)$ & $107.7(2)$ \\
\hline $\mathrm{C}(14)-\mathrm{C}(13)-\mathrm{C}(12)$ & 118.7(3) & $\mathrm{C}(32)-\mathrm{C}(31)-\mathrm{P}(2)$ & $109.4(2)$ \\
\hline $\mathrm{C}(14)-\mathrm{C}(13)-\mathrm{C}(30)$ & 120.7(3) & $C(37)-C(35)-C(36)$ & 109.5(3) \\
\hline $\mathrm{C}(12)-\mathrm{C}(13)-\mathrm{C}(30)$ & $120.4(3)$ & $\mathrm{C}(37)-\mathrm{C}(35)-\mathrm{C}(38)$ & $107.4(3)$ \\
\hline $\mathrm{C}(13)-\mathrm{C}(14)-\mathrm{C}(15)$ & 121.9(3) & $\mathrm{C}(36)-\mathrm{C}(35)-\mathrm{C}(38)$ & 108.7(3) \\
\hline $\mathrm{C}(14)-\mathrm{C}(15)-\mathrm{C}(20)$ & 118.8(3) & $\mathrm{C}(37)-\mathrm{C}(35)-\mathrm{P}(2)$ & $112.5(3)$ \\
\hline $\mathrm{C}(14)-\mathrm{C}(15)-\mathrm{C}(16)$ & 122.2(3) & $\mathrm{C}(36)-\mathrm{C}(35)-\mathrm{P}(2)$ & $112.7(2)$ \\
\hline $\mathrm{C}(20)-\mathrm{C}(15)-\mathrm{C}(16)$ & 119.0(3) & $\mathrm{C}(38)-\mathrm{C}(35)-\mathrm{P}(2)$ & $105.8(2)$ \\
\hline
\end{tabular}




\begin{tabular}{|c|c|c|c|}
\hline$C(40)-C(39)-C(48)$ & 120.2(3) & $\mathrm{C}(62)-\mathrm{C}(60)-\mathrm{P}(3)$ & $113.9(2)$ \\
\hline$C(40)-C(39)-C(49)$ & 119.8(3) & $\mathrm{C}(63)-\mathrm{C}(60)-\mathrm{P}(3)$ & 108.3(2) \\
\hline C(48)-C(39)-C(49) & 119.9(3) & $\mathrm{C}(66)-\mathrm{C}(64)-\mathrm{C}(65)$ & 106.9(3) \\
\hline $\mathrm{O}(5)-\mathrm{C}(40)-\mathrm{C}(39)$ & $118.6(2)$ & $\mathrm{C}(66)-\mathrm{C}(64)-\mathrm{C}(67)$ & 110.1(3) \\
\hline $\mathrm{O}(5)-\mathrm{C}(40)-\mathrm{C}(41)$ & 119.7(3) & $\mathrm{C}(65)-\mathrm{C}(64)-\mathrm{C}(67)$ & 108.4(3) \\
\hline$C(39)-C(40)-C(41)$ & $121.6(3)$ & $\mathrm{C}(66)-\mathrm{C}(64)-\mathrm{P}(3)$ & $112.2(3)$ \\
\hline$C(42)-C(41)-C(40)$ & 117.9(3) & $\mathrm{C}(65)-\mathrm{C}(64)-\mathrm{P}(3)$ & $106.0(2)$ \\
\hline$C(42)-C(41)-C(59)$ & 121.6(3) & $\mathrm{C}(67)-\mathrm{C}(64)-\mathrm{P}(3)$ & $112.9(2)$ \\
\hline$C(40)-C(41)-C(59)$ & 120.3(3) & $\mathrm{C}(51)-\mathrm{C}(68)-\mathrm{P}(4)$ & $120.0(2)$ \\
\hline$C(41)-C(42)-C(43)$ & $122.6(3)$ & $\mathrm{C}(70)-\mathrm{C}(69)-\mathrm{C}(71)$ & $108.8(4)$ \\
\hline$C(42)-C(43)-C(44)$ & $122.4(3)$ & C(70)-C(69)-C(72) & $110.7(5)$ \\
\hline$C(42)-C(43)-C(48)$ & 118.9(3) & C(71)-C(69)-C(72) & 106.3(4) \\
\hline$C(44)-C(43)-C(48)$ & 118.7(3) & $\mathrm{C}(70)-\mathrm{C}(69)-\mathrm{P}(4)$ & 113.2(3) \\
\hline$C(45)-C(44)-C(43)$ & 121.6(3) & $\mathrm{C}(71)-\mathrm{C}(69)-\mathrm{P}(4)$ & $112.4(3)$ \\
\hline$C(44)-C(45)-C(46)$ & 119.4(3) & $\mathrm{C}(72)-\mathrm{C}(69)-\mathrm{P}(4)$ & $105.2(3)$ \\
\hline$C(47)-C(46)-C(45)$ & $120.8(4)$ & C(76)-C(73)-C(75) & $109.1(4)$ \\
\hline$C(46)-C(47)-C(48)$ & $121.5(3)$ & $\mathrm{C}(76)-\mathrm{C}(73)-\mathrm{C}(74)$ & $108.5(5)$ \\
\hline$C(47)-C(48)-C(43)$ & 118.1(3) & C(75)-C(73)-C(74) & 109.3(4) \\
\hline $\mathrm{C}(47)-\mathrm{C}(48)-\mathrm{C}(39)$ & 123.3(3) & $\mathrm{C}(76)-\mathrm{C}(73)-\mathrm{P}(4)$ & $106.0(3)$ \\
\hline C(43)-C(48)-C(39) & 118.6(3) & $\mathrm{C}(75)-\mathrm{C}(73)-\mathrm{P}(4)$ & 114.1(3) \\
\hline$C(50)-C(49)-C(58)$ & $120.2(2)$ & $\mathrm{C}(74)-\mathrm{C}(73)-\mathrm{P}(4)$ & 109.6(3) \\
\hline$C(50)-C(49)-C(39)$ & $119.8(2)$ & $\mathrm{C}(78)-\mathrm{C}(77)-\mathrm{C}(83)$ & 133.5(10) \\
\hline C(58)-C(49)-C(39) & $120.1(2)$ & C(78)-C(77)-C(82) & $114.0(6)$ \\
\hline $\mathrm{O}(6)-\mathrm{C}(50)-\mathrm{C}(49)$ & $117.9(2)$ & $\mathrm{C}(83)-\mathrm{C}(77)-\mathrm{C}(82)$ & $112.5(9)$ \\
\hline $\mathrm{O}(6)-\mathrm{C}(50)-\mathrm{C}(51)$ & 121.5(3) & C(79)-C(78)-C(77) & $135.8(11)$ \\
\hline$C(49)-C(50)-C(51)$ & 120.6(3) & C(78)-C(79)-C(80) & 109.1(12) \\
\hline$C(52)-C(51)-C(50)$ & 118.6(3) & C(81)-C(80)-C(79) & 127.9(9) \\
\hline$C(52)-C(51)-C(68)$ & $120.0(2)$ & $\mathrm{C}(80)-\mathrm{C}(81)-\mathrm{C}(82)$ & $120.4(8)$ \\
\hline$C(50)-C(51)-C(68)$ & $121.0(2)$ & C(81)-C(82)-C(77) & $112.7(7)$ \\
\hline$C(51)-C(52)-C(53)$ & 122.3(3) & C(89)-C(84)-C(85) & 116.1(7) \\
\hline C(58)-C(53)-C(52) & 118.7(3) & C(89)-C(84)-C(90) & $122.0(8)$ \\
\hline$C(58)-C(53)-C(54)$ & 119.3(3) & $\mathrm{C}(85)-\mathrm{C}(84)-\mathrm{C}(90)$ & $121.5(9)$ \\
\hline$C(52)-C(53)-C(54)$ & $122.0(3)$ & C(86)-C(85)-C(84) & 123.1(9) \\
\hline$C(55)-C(54)-C(53)$ & 121.0(3) & C(87)-C(86)-C(85) & 113.0(10) \\
\hline$C(54)-C(55)-C(56)$ & 120.1(3) & $\mathrm{C}(86)-\mathrm{C}(87)-\mathrm{C}(88)$ & $127.3(11)$ \\
\hline$C(57)-C(56)-C(55)$ & $120.4(3)$ & C(89)-C(88)-C(87) & 119.9(9) \\
\hline$C(56)-C(57)-C(58)$ & 121.0(3) & C(88)-C(89)-C(84) & $120.3(7)$ \\
\hline C(53)-C(58)-C(49) & 119.6(3) & $\mathrm{O}(3)-\mathrm{P}(1)-\mathrm{C}(21)$ & $111.17(13)$ \\
\hline $\mathrm{C}(53)-\mathrm{C}(58)-\mathrm{C}(57)$ & 118.1(3) & $\mathrm{O}(3)-\mathrm{P}(1)-\mathrm{C}(26)$ & $110.42(15)$ \\
\hline C(49)-C(58)-C(57) & $122.3(3)$ & $\mathrm{C}(21)-\mathrm{P}(1)-\mathrm{C}(26)$ & 103.64(15) \\
\hline C(41)-C(59)-P(3) & $117.9(2)$ & $\mathrm{O}(3)-\mathrm{P}(1)-\mathrm{C}(22)$ & 108.48(13) \\
\hline$C(61)-C(60)-C(62)$ & 108.9(3) & $\mathrm{C}(21)-\mathrm{P}(1)-\mathrm{C}(22)$ & $109.27(14)$ \\
\hline$C(61)-C(60)-C(63)$ & 108.7(3) & $\mathrm{C}(26)-\mathrm{P}(1)-\mathrm{C}(22)$ & 113.84(16) \\
\hline$C(62)-C(60)-C(63)$ & 109.6(3) & $\mathrm{O}(4)-\mathrm{P}(2)-\mathrm{C}(30)$ & $111.40(13)$ \\
\hline $\mathrm{C}(61)-\mathrm{C}(60)-\mathrm{P}(3)$ & $107.2(2)$ & $\mathrm{O}(4)-\mathrm{P}(2)-\mathrm{C}(31)$ & $110.19(14)$ \\
\hline
\end{tabular}




$\begin{array}{llll}\mathrm{C}(30)-\mathrm{P}(2)-\mathrm{C}(31) & 108.57(15) & \mathrm{C}(59)-\mathrm{P}(3)-\mathrm{C}(60) & 108.78(15) \\ \mathrm{O}(4)-\mathrm{P}(2)-\mathrm{C}(35) & 109.61(15) & \mathrm{C}(64)-\mathrm{P}(3)-\mathrm{C}(60) & 113.28(16) \\ \mathrm{C}(30)-\mathrm{P}(2)-\mathrm{C}(35) & 103.57(15) & \mathrm{O}(8)-\mathrm{P}(4)-\mathrm{C}(68) & 110.85(13) \\ \mathrm{C}(31)-\mathrm{P}(2)-\mathrm{C}(35) & 113.35(17) & \mathrm{O}(8)-\mathrm{P}(4)-\mathrm{C}(73) & 108.59(15) \\ \mathrm{O}(7)-\mathrm{P}(3)-\mathrm{C}(59) & 110.85(13) & \mathrm{C}(68)-\mathrm{P}(4)-\mathrm{C}(73) & 104.18(16) \\ \mathrm{O}(7)-\mathrm{P}(3)-\mathrm{C}(64) & 109.59(14) & \mathrm{O}(8)-\mathrm{P}(4)-\mathrm{C}(69) & 108.89(14) \\ \mathrm{C}(59)-\mathrm{P}(3)-\mathrm{C}(64) & 103.90(16) & \mathrm{C}(68)-\mathrm{P}(4)-\mathrm{C}(69) & 108.84(16) \\ \mathrm{O}(7)-\mathrm{P}(3)-\mathrm{C}(60) & 110.29(14) & \mathrm{C}(73)-\mathrm{P}(4)-\mathrm{C}(69) & 115.42(18)\end{array}$

Symmetry transformations used to generate equivalent atoms: 
Table S14. Anisotropic displacement parameters $\left(\AA^{2} \times 10^{3}\right)$ for 3 . The anisotropic displacement factor exponent takes the form: $-2 \pi^{2}\left[\mathrm{~h}^{2} \mathrm{a}^{* 2} \mathrm{U}^{11}+\ldots+2 \mathrm{hka} \mathrm{b}^{*} \mathrm{U}^{12}\right]$

\begin{tabular}{lcccccc}
\hline & $\mathrm{U}^{11}$ & $\mathrm{U}^{22}$ & $\mathrm{U}^{33}$ & $\mathrm{U}^{23}$ & $\mathrm{U}^{13}$ & $\mathrm{U}^{12}$ \\
\hline $\mathrm{C}(1)$ & $28(1)$ & $30(1)$ & $35(1)$ & $17(1)$ & $4(1)$ & $-4(1)$ \\
$\mathrm{C}(2)$ & $30(1)$ & $29(1)$ & $37(2)$ & $19(1)$ & $3(1)$ & $0(1)$ \\
$\mathrm{C}(3)$ & $33(2)$ & $30(1)$ & $36(1)$ & $17(1)$ & $8(1)$ & $0(1)$ \\
$\mathrm{C}(4)$ & $39(2)$ & $31(2)$ & $45(2)$ & $22(1)$ & $9(1)$ & $-1(1)$ \\
$\mathrm{C}(5)$ & $39(2)$ & $31(2)$ & $40(2)$ & $17(1)$ & $9(1)$ & $5(1)$ \\
$\mathrm{C}(6)$ & $53(2)$ & $36(2)$ & $54(2)$ & $25(2)$ & $14(2)$ & $9(2)$ \\
$\mathrm{C}(7)$ & $56(2)$ & $42(2)$ & $52(2)$ & $20(2)$ & $12(2)$ & $23(2)$ \\
$\mathrm{C}(8)$ & $37(2)$ & $50(2)$ & $41(2)$ & $12(2)$ & $7(1)$ & $10(2)$ \\
$\mathrm{C}(9)$ & $32(2)$ & $41(2)$ & $37(2)$ & $13(1)$ & $5(1)$ & $-1(1)$ \\
$\mathrm{C}(10)$ & $31(1)$ & $31(1)$ & $34(1)$ & $12(1)$ & $5(1)$ & $1(1)$ \\
$\mathrm{C}(11)$ & $23(1)$ & $29(1)$ & $42(2)$ & $16(1)$ & $8(1)$ & $1(1)$ \\
$\mathrm{C}(12)$ & $32(2)$ & $28(1)$ & $42(2)$ & $17(1)$ & $8(1)$ & $-1(1)$ \\
$\mathrm{C}(13)$ & $36(2)$ & $29(1)$ & $42(2)$ & $18(1)$ & $14(1)$ & $4(1)$ \\
$\mathrm{C}(14)$ & $39(2)$ & $30(2)$ & $48(2)$ & $20(1)$ & $17(1)$ & $-1(1)$ \\
$\mathrm{C}(15)$ & $29(2)$ & $30(2)$ & $52(2)$ & $16(1)$ & $11(1)$ & $1(1)$ \\
$\mathrm{C}(16)$ & $40(2)$ & $32(2)$ & $62(2)$ & $14(2)$ & $13(2)$ & $-4(1)$ \\
$\mathrm{C}(17)$ & $45(2)$ & $43(2)$ & $61(2)$ & $7(2)$ & $2(2)$ & $-12(2)$ \\
$\mathrm{C}(18)$ & $45(2)$ & $51(2)$ & $43(2)$ & $15(2)$ & $2(1)$ & $1(2)$ \\
$\mathrm{C}(19)$ & $39(2)$ & $40(2)$ & $43(2)$ & $18(1)$ & $6(1)$ & $2(1)$ \\
$\mathrm{C}(20)$ & $27(1)$ & $29(1)$ & $45(2)$ & $18(1)$ & $8(1)$ & $2(1)$ \\
$\mathrm{C}(21)$ & $32(2)$ & $28(1)$ & $44(2)$ & $15(1)$ & $10(1)$ & $-1(1)$ \\
$\mathrm{C}(22)$ & $39(2)$ & $29(2)$ & $42(2)$ & $16(1)$ & $6(1)$ & $3(1)$ \\
$\mathrm{C}(23)$ & $40(2)$ & $111(4)$ & $56(2)$ & $29(2)$ & $-2(2)$ & $6(2)$ \\
$\mathrm{C}(24)$ & $81(3)$ & $42(2)$ & $62(2)$ & $32(2)$ & $-5(2)$ & $-8(2)$ \\
$\mathrm{C}(25)$ & $77(3)$ & $46(2)$ & $42(2)$ & $16(2)$ & $4(2)$ & $11(2)$ \\
$\mathrm{C}(26)$ & $22(1)$ & $48(2)$ & $61(2)$ & $17(2)$ & $9(1)$ & $0(1)$ \\
$\mathrm{C}(27)$ & $35(2)$ & $58(2)$ & $93(3)$ & $32(2)$ & $8(2)$ & $-11(2)$ \\
$\mathrm{C}(28)$ & $42(2)$ & $62(3)$ & $84(3)$ & $15(2)$ & $25(2)$ & $11(2)$ \\
$\mathrm{C}(29)$ & $33(2)$ & $93(3)$ & $74(3)$ & $49(2)$ & $0(2)$ & $12(2)$ \\
$\mathrm{C}(30)$ & $38(2)$ & $28(1)$ & $42(2)$ & $20(1)$ & $12(1)$ & $3(1)$ \\
$\mathrm{C}(31)$ & $37(2)$ & $38(2)$ & $61(2)$ & $25(2)$ & $21(2)$ & $10(1)$ \\
$\mathrm{C}(32)$ & $49(2)$ & $44(2)$ & $60(2)$ & $27(2)$ & $21(2)$ & $15(2)$ \\
$\mathrm{C}(33)$ & $55(2)$ & $44(2)$ & $82(3)$ & $35(2)$ & $32(2)$ & $12(2)$ \\
$\mathrm{C}(34)$ & $36(2)$ & $51(2)$ & $64(2)$ & $16(2)$ & $10(2)$ & $6(2)$ \\
$\mathrm{C}(35)$ & $55(2)$ & $36(2)$ & $41(2)$ & $17(1)$ & $1(2)$ & $9(2)$ \\
$\mathrm{C}(36)$ & $75(3)$ & $43(2)$ & $54(2)$ & $26(2)$ & $-1(2)$ & $12(2)$ \\
$\mathrm{C}(37)$ & $76(3)$ & $43(2)$ & $43(2)$ & $14(2)$ & $9(2)$ & $9(2)$ \\
$\mathrm{C}(38)$ & $49(2)$ & $43(2)$ & $61(2)$ & $21(2)$ & $-4(2)$ & $0(2)$ \\
$\mathrm{C}(39)$ & $24(1)$ & $27(1)$ & $46(2)$ & $17(1)$ & $1(1)$ & $1(1)$ \\
$\mathrm{C}(40)$ & $27(1)$ & $30(1)$ & $45(2)$ & $19(1)$ & $2(1)$ & $2(1)$ \\
& & & & & & \\
& & & & 29 & &
\end{tabular}




\begin{tabular}{|c|c|c|c|c|c|c|}
\hline $\mathrm{C}(41)$ & $32(2)$ & $29(2)$ & $45(2)$ & $19(1)$ & $-2(1)$ & $0(1)$ \\
\hline$C(42)$ & $34(2)$ & $26(1)$ & $54(2)$ & $17(1)$ & $-5(1)$ & $4(1)$ \\
\hline$C(43)$ & $32(2)$ & $27(2)$ & $52(2)$ & $13(1)$ & $-4(1)$ & $2(1)$ \\
\hline$C(44)$ & $43(2)$ & $33(2)$ & $64(2)$ & $12(2)$ & $-2(2)$ & $7(1)$ \\
\hline$C(45)$ & $52(2)$ & $41(2)$ & $64(2)$ & $1(2)$ & $8(2)$ & $9(2)$ \\
\hline$C(46)$ & $49(2)$ & $46(2)$ & $48(2)$ & $12(2)$ & $7(2)$ & $1(2)$ \\
\hline $\mathrm{C}(47)$ & $35(2)$ & $35(2)$ & $49(2)$ & $16(1)$ & $2(1)$ & 1(1) \\
\hline $\mathrm{C}(48)$ & $25(1)$ & $28(1)$ & $48(2)$ & $16(1)$ & $0(1)$ & 1(1) \\
\hline$C(49)$ & $31(1)$ & $29(1)$ & $36(1)$ & $17(1)$ & $5(1)$ & $5(1)$ \\
\hline$C(50)$ & $32(2)$ & $27(1)$ & $37(2)$ & $17(1)$ & $6(1)$ & $4(1)$ \\
\hline $\mathrm{C}(51)$ & $27(1)$ & $29(1)$ & $41(2)$ & $18(1)$ & $4(1)$ & $4(1)$ \\
\hline$C(52)$ & $34(2)$ & $33(2)$ & $45(2)$ & $23(1)$ & $2(1)$ & $5(1)$ \\
\hline C(53) & $36(2)$ & $31(2)$ & $44(2)$ & $20(1)$ & 1(1) & $0(1)$ \\
\hline$C(54)$ & $43(2)$ & $38(2)$ & $59(2)$ & $28(2)$ & $1(2)$ & $-2(1)$ \\
\hline C(55) & $42(2)$ & $42(2)$ & $56(2)$ & $24(2)$ & $3(2)$ & $-8(2)$ \\
\hline$C(56)$ & $32(2)$ & $44(2)$ & $45(2)$ & $14(1)$ & $0(1)$ & $-5(1)$ \\
\hline $\mathrm{C}(57)$ & $32(2)$ & $35(2)$ & $41(2)$ & $15(1)$ & $2(1)$ & $4(1)$ \\
\hline $\mathrm{C}(58)$ & $32(2)$ & $29(1)$ & $33(1)$ & $12(1)$ & $3(1)$ & $3(1)$ \\
\hline C(59) & $36(2)$ & $31(2)$ & $52(2)$ & $25(1)$ & $2(1)$ & 1(1) \\
\hline $\mathrm{C}(60)$ & $36(2)$ & $34(2)$ & $45(2)$ & $18(1)$ & $3(1)$ & 1(1) \\
\hline $\mathrm{C}(61)$ & $36(2)$ & $47(2)$ & $56(2)$ & $22(2)$ & $11(2)$ & 1(1) \\
\hline$C(62)$ & $42(2)$ & $44(2)$ & $54(2)$ & $26(2)$ & $2(2)$ & $6(1)$ \\
\hline C(63) & $41(2)$ & $43(2)$ & $53(2)$ & $19(2)$ & $1(2)$ & $-7(1)$ \\
\hline $\mathrm{C}(64)$ & $57(2)$ & $32(2)$ & $47(2)$ & $20(1)$ & $19(2)$ & $2(1)$ \\
\hline $\mathrm{C}(65)$ & $53(2)$ & $41(2)$ & $80(3)$ & $24(2)$ & $34(2)$ & $8(2)$ \\
\hline $\mathrm{C}(66)$ & $82(3)$ & $42(2)$ & $44(2)$ & $16(2)$ & $18(2)$ & $3(2)$ \\
\hline $\mathrm{C}(67)$ & 91(3) & $39(2)$ & $66(2)$ & $30(2)$ & $37(2)$ & $4(2)$ \\
\hline C(68) & $28(2)$ & $28(1)$ & $49(2)$ & $15(1)$ & $7(1)$ & $6(1)$ \\
\hline C(69) & $57(2)$ & $32(2)$ & $45(2)$ & $18(1)$ & $6(2)$ & $3(2)$ \\
\hline $\mathrm{C}(70)$ & 197(7) & $48(3)$ & $94(4)$ & $48(3)$ & $79(4)$ & $42(3)$ \\
\hline $\mathrm{C}(71)$ & $150(6)$ & $75(3)$ & $47(2)$ & $10(2)$ & $10(3)$ & $-42(3)$ \\
\hline$C(72)$ & $78(3)$ & 153(5) & 61(3) & $35(3)$ & 41(3) & $27(4)$ \\
\hline $\mathrm{C}(73)$ & $26(2)$ & $40(2)$ & $88(3)$ & $22(2)$ & $9(2)$ & $4(1)$ \\
\hline $\mathrm{C}(74)$ & $33(2)$ & $53(3)$ & 197(7) & $-12(3)$ & $9(3)$ & $-7(2)$ \\
\hline$C(75)$ & $39(2)$ & $50(2)$ & $122(4)$ & $30(2)$ & $14(2)$ & $14(2)$ \\
\hline$C(76)$ & $47(3)$ & $160(6)$ & $146(5)$ & $98(5)$ & $50(3)$ & $24(3)$ \\
\hline $\mathrm{C}(77)$ & $56(3)$ & 187(7) & $77(4)$ & $77(4)$ & 23(3) & $10(4)$ \\
\hline$C(78)$ & 173(8) & $83(4)$ & $83(4)$ & $19(3)$ & $62(5)$ & $0(4)$ \\
\hline $\mathrm{C}(79)$ & $76(5)$ & $316(16)$ & 121(7) & $86(8)$ & $18(5)$ & $-24(7)$ \\
\hline $\mathrm{C}(80)$ & $77(4)$ & $143(7)$ & $144(7)$ & $69(6)$ & $56(4)$ & $22(5)$ \\
\hline $\mathrm{C}(81)$ & $86(5)$ & 113(5) & 134(6) & $62(5)$ & $52(4)$ & $18(4)$ \\
\hline $\mathrm{C}(82)$ & 139(7) & $132(6)$ & 119(5) & $42(5)$ & $90(5)$ & $-3(5)$ \\
\hline C(83) & 213(12) & $370(18)$ & $87(5)$ & $12(8)$ & $55(7)$ & $-152(13)$ \\
\hline$C(84)$ & $84(4)$ & $129(6)$ & $96(4)$ & $9(4)$ & $9(4)$ & $-32(4)$ \\
\hline$C(85)$ & $81(4)$ & 210(9) & 132(6) & $120(7)$ & $44(5)$ & $49(5)$ \\
\hline
\end{tabular}




\begin{tabular}{lcccccc}
$\mathrm{C}(86)$ & $151(8)$ & $173(8)$ & $77(4)$ & $29(5)$ & $35(5)$ & $-78(7)$ \\
$\mathrm{C}(87)$ & $88(5)$ & $197(9)$ & $108(6)$ & $79(6)$ & $37(4)$ & $3(5)$ \\
$\mathrm{C}(88)$ & $67(4)$ & $186(8)$ & $80(4)$ & $35(5)$ & $13(3)$ & $-30(4)$ \\
$\mathrm{C}(89)$ & $64(3)$ & $160(7)$ & $111(5)$ & $74(5)$ & $13(3)$ & $24(4)$ \\
$\mathrm{C}(90)$ & $149(8)$ & $321(15)$ & $98(6)$ & $-20(8)$ & $-3(6)$ & $114(10)$ \\
$\mathrm{O}(1)$ & $36(1)$ & $35(1)$ & $51(1)$ & $26(1)$ & $14(1)$ & $8(1)$ \\
$\mathrm{O}(2)$ & $46(1)$ & $39(1)$ & $35(1)$ & $15(1)$ & $5(1)$ & $-12(1)$ \\
$\mathrm{O}(3)$ & $37(1)$ & $30(1)$ & $46(1)$ & $18(1)$ & $11(1)$ & $3(1)$ \\
$\mathrm{O}(4)$ & $49(1)$ & $31(1)$ & $48(1)$ & $25(1)$ & $13(1)$ & $9(1)$ \\
$\mathrm{O}(5)$ & $43(1)$ & $34(1)$ & $45(1)$ & $16(1)$ & $7(1)$ & $13(1)$ \\
$\mathrm{O}(6)$ & $33(1)$ & $37(1)$ & $54(1)$ & $30(1)$ & $5(1)$ & $2(1)$ \\
$\mathrm{O}(7)$ & $45(1)$ & $33(1)$ & $50(1)$ & $25(1)$ & $6(1)$ & $0(1)$ \\
$\mathrm{O}(8)$ & $35(1)$ & $28(1)$ & $54(1)$ & $21(1)$ & $4(1)$ & $4(1)$ \\
$\mathrm{P}(1)$ & $27(1)$ & $30(1)$ & $43(1)$ & $17(1)$ & $7(1)$ & $1(1)$ \\
$\mathrm{P}(2)$ & $40(1)$ & $27(1)$ & $40(1)$ & $19(1)$ & $9(1)$ & $6(1)$ \\
$\mathrm{P}(3)$ & $36(1)$ & $28(1)$ & $41(1)$ & $19(1)$ & $7(1)$ & $1(1)$ \\
$\mathrm{P}(4)$ & $27(1)$ & $26(1)$ & $49(1)$ & $17(1)$ & $5(1)$ & $4(1)$ \\
& & & & & & \\
\hline
\end{tabular}


Table S15. Hydrogen coordinates $\left(\times 10^{4}\right)$ and isotropic displacement parameters $\left(\AA^{2} \times 10^{3}\right)$ for 3.

\begin{tabular}{|c|c|c|c|c|}
\hline & $\mathrm{x}$ & $\mathrm{y}$ & $\mathrm{z}$ & $\mathrm{U}(\mathrm{eq})$ \\
\hline $\mathrm{H}(4)$ & 7060 & 4968 & 8555 & 43 \\
\hline $\mathrm{H}(6)$ & 8758 & 3738 & 8284 & 54 \\
\hline $\mathrm{H}(7)$ & 10537 & 3457 & 7829 & 58 \\
\hline $\mathrm{H}(8)$ & 11360 & 4900 & 7331 & 52 \\
\hline $\mathrm{H}(9)$ & 10359 & 6543 & 7231 & 44 \\
\hline $\mathrm{H}(14)$ & 10084 & 10392 & 6537 & 43 \\
\hline $\mathrm{H}(16)$ & 11135 & 11550 & 8058 & 53 \\
\hline $\mathrm{H}(17)$ & 11486 & 11846 & 9544 & 64 \\
\hline $\mathrm{H}(18)$ & 10614 & 10492 & 10082 & 57 \\
\hline $\mathrm{H}(19)$ & 9416 & 8841 & 9142 & 48 \\
\hline $\mathrm{H}(21 \mathrm{~A})$ & 5386 & 5880 & 8689 & 40 \\
\hline $\mathrm{H}(21 \mathrm{~B})$ & 4976 & 6500 & 7957 & 40 \\
\hline $\mathrm{H}(23 \mathrm{~A})$ & 7530 & 8448 & 10223 & 105 \\
\hline $\mathrm{H}(23 \mathrm{~B})$ & 7394 & 7037 & 10059 & 105 \\
\hline $\mathrm{H}(23 \mathrm{C})$ & 7714 & 7950 & 11039 & 105 \\
\hline $\mathrm{H}(24 \mathrm{~A})$ & 5955 & 6866 & 11275 & 92 \\
\hline $\mathrm{H}(24 \mathrm{~B})$ & 5647 & 6077 & 10260 & 92 \\
\hline $\mathrm{H}(24 \mathrm{C})$ & 4662 & 6849 & 10691 & 92 \\
\hline $\mathrm{H}(25 \mathrm{~A})$ & 4983 & 9078 & 11227 & 83 \\
\hline $\mathrm{H}(25 \mathrm{~B})$ & 5989 & 9740 & 10950 & 83 \\
\hline $\mathrm{H}(25 \mathrm{C})$ & 6348 & 9123 & 11696 & 83 \\
\hline $\mathrm{H}(27 \mathrm{~A})$ & 3420 & 6216 & 9565 & 91 \\
\hline $\mathrm{H}(27 \mathrm{~B})$ & 3332 & 5860 & 8530 & 91 \\
\hline $\mathrm{H}(27 \mathrm{C})$ & 2216 & 6358 & 8938 & 91 \\
\hline $\mathrm{H}(28 \mathrm{~A})$ & 3417 & 9381 & 10024 & 94 \\
\hline $\mathrm{H}(28 \mathrm{~B})$ & 3305 & 8324 & 10430 & 94 \\
\hline $\mathrm{H}(28 \mathrm{C})$ & 2207 & 8542 & 9744 & 94 \\
\hline $\mathrm{H}(29 \mathrm{~A})$ & 2191 & 7955 & 8188 & 95 \\
\hline H(29B) & 3323 & 7431 & 7814 & 95 \\
\hline $\mathrm{H}(29 \mathrm{C})$ & 3365 & 8817 & 8373 & 95 \\
\hline $\mathrm{H}(30 \mathrm{~A})$ & 9073 & 9432 & 5128 & 40 \\
\hline $\mathrm{H}(30 \mathrm{~B})$ & 7904 & 8617 & 5028 & 40 \\
\hline $\mathrm{H}(32 \mathrm{~A})$ & 11041 & 6453 & 3323 & 71 \\
\hline H(32B) & 11366 & 6117 & 4209 & 71 \\
\hline $\mathrm{H}(32 \mathrm{C})$ & 12285 & 6954 & 3991 & 71 \\
\hline $\mathrm{H}(33 \mathrm{~A})$ & 10493 & 9489 & 4218 & 81 \\
\hline H(33B) & 10568 & 8457 & 3331 & 81 \\
\hline H(33C) & 11759 & 9084 & 4026 & 81 \\
\hline $\mathrm{H}(34 \mathrm{~A})$ & 12420 & 8466 & 5371 & 76 \\
\hline $\mathrm{H}(34 \mathrm{~B})$ & 11450 & 7823 & 5693 & 76 \\
\hline
\end{tabular}




\begin{tabular}{|c|c|c|c|c|}
\hline $\mathrm{H}(34 \mathrm{C})$ & 11297 & 9151 & 5647 & 76 \\
\hline $\mathrm{H}(36 \mathrm{~A})$ & 8621 & 8787 & 3228 & 85 \\
\hline $\mathrm{H}(36 \mathrm{~B})$ & 7511 & 8778 & 3680 & 85 \\
\hline $\mathrm{H}(36 \mathrm{C})$ & 7370 & 8122 & 2649 & 85 \\
\hline $\mathrm{H}(37 \mathrm{~A})$ & 8100 & 6109 & 2096 & 82 \\
\hline H(37B) & 9023 & 5623 & 2751 & 82 \\
\hline $\mathrm{H}(37 \mathrm{C})$ & 9399 & 6778 & 2519 & 82 \\
\hline $\mathrm{H}(38 \mathrm{~A})$ & 6543 & 6356 & 2947 & 79 \\
\hline H(38B) & 6877 & 6941 & 3991 & 79 \\
\hline $\mathrm{H}(38 \mathrm{C})$ & 7370 & 5693 & 3530 & 79 \\
\hline $\mathrm{H}(42)$ & 5358 & -155 & 7381 & 46 \\
\hline $\mathrm{H}(44)$ & 5623 & -1295 & 5890 & 59 \\
\hline $\mathrm{H}(45)$ & 5230 & -1592 & 4408 & 68 \\
\hline $\mathrm{H}(46)$ & 4128 & -222 & 3843 & 59 \\
\hline $\mathrm{H}(47)$ & 3462 & 1408 & 4760 & 48 \\
\hline H(52) & 1420 & 5291 & 5372 & 43 \\
\hline $\mathrm{H}(54)$ & 3327 & 6473 & 5604 & 54 \\
\hline $\mathrm{H}(55)$ & 5371 & 6678 & 5988 & 55 \\
\hline $\mathrm{H}(56)$ & 6417 & 5202 & 6437 & 50 \\
\hline $\mathrm{H}(57)$ & 5405 & 3593 & 6577 & 43 \\
\hline $\mathrm{H}(59 \mathrm{~A})$ & 5069 & 790 & 8789 & 46 \\
\hline H(59B) & 3945 & 1563 & 8842 & 46 \\
\hline $\mathrm{H}(61 \mathrm{~A})$ & 8334 & 1797 & 8736 & 67 \\
\hline $\mathrm{H}(61 \mathrm{~B})$ & 7182 & 2351 & 8336 & 67 \\
\hline $\mathrm{H}(61 \mathrm{C})$ & 7067 & 1049 & 8437 & 67 \\
\hline $\mathrm{H}(62 \mathrm{~A})$ & 6956 & 827 & 9877 & 68 \\
\hline $\mathrm{H}(62 \mathrm{~B})$ & 7479 & 1921 & 10747 & 68 \\
\hline $\mathrm{H}(62 \mathrm{C})$ & 8333 & 1282 & 10111 & 68 \\
\hline $\mathrm{H}(63 \mathrm{~A})$ & 8888 & 3411 & 10059 & 69 \\
\hline $\mathrm{H}(63 \mathrm{~B})$ & 7985 & 3901 & 10691 & 69 \\
\hline $\mathrm{H}(63 \mathrm{C})$ & 7829 & 4169 & 9779 & 69 \\
\hline $\mathrm{H}(65 \mathrm{~A})$ & 3557 & 3759 & 10843 & 81 \\
\hline H(65B) & 3402 & 3135 & 9808 & 81 \\
\hline $\mathrm{H}(65 \mathrm{C})$ & 4100 & 4429 & 10274 & 81 \\
\hline $\mathrm{H}(66 \mathrm{~A})$ & 6096 & 4684 & 11170 & 82 \\
\hline $\mathrm{H}(66 \mathrm{~B})$ & 6671 & 3589 & 11439 & 82 \\
\hline $\mathrm{H}(66 \mathrm{C})$ & 5539 & 4139 & 11801 & 82 \\
\hline $\mathrm{H}(67 \mathrm{~A})$ & 5573 & 1510 & 10787 & 88 \\
\hline $\mathrm{H}(67 \mathrm{~B})$ & 4269 & 1357 & 10184 & 88 \\
\hline $\mathrm{H}(67 \mathrm{C})$ & 4491 & 2083 & 11210 & 88 \\
\hline $\mathrm{H}(68 \mathrm{~A})$ & -370 & 4337 & 5184 & 41 \\
\hline H(68B) & -404 & 3675 & 5889 & 41 \\
\hline $\mathrm{H}(70 \mathrm{~A})$ & -562 & 3401 & 2680 & 150 \\
\hline $\mathrm{H}(70 \mathrm{~B})$ & -374 & 4119 & 3706 & 150 \\
\hline $\mathrm{H}(70 \mathrm{C})$ & -1671 & 3560 & 3149 & 150 \\
\hline
\end{tabular}




\begin{tabular}{|c|c|c|c|c|}
\hline $\mathrm{H}(71 \mathrm{~A})$ & -2046 & 1490 & 2450 & 142 \\
\hline $\mathrm{H}(71 \mathrm{~B})$ & -1361 & 566 & 2870 & 142 \\
\hline $\mathrm{H}(71 \mathrm{C})$ & -817 & 1046 & 2202 & 142 \\
\hline $\mathrm{H}(72 \mathrm{~A})$ & 950 & 1995 & 2960 & 140 \\
\hline $\mathrm{H}(72 \mathrm{~B})$ & 847 & 1322 & 3656 & 140 \\
\hline $\mathrm{H}(72 \mathrm{C})$ & 1193 & 2734 & 3984 & 140 \\
\hline $\mathrm{H}(74 \mathrm{~A})$ & -2964 & 710 & 3828 & 163 \\
\hline $\mathrm{H}(74 \mathrm{~B})$ & -3349 & 1596 & 3273 & 163 \\
\hline $\mathrm{H}(74 \mathrm{C})$ & -4098 & 1459 & 3967 & 163 \\
\hline $\mathrm{H}(75 \mathrm{~A})$ & -3726 & 3771 & 4635 & 105 \\
\hline $\mathrm{H}(75 \mathrm{~B})$ & -2962 & 3724 & 3912 & 105 \\
\hline $\mathrm{H}(75 \mathrm{C})$ & -2383 & 4346 & 4930 & 105 \\
\hline $\mathrm{H}(76 \mathrm{~A})$ & -2203 & 3119 & 5928 & 153 \\
\hline $\mathrm{H}(76 \mathrm{~B})$ & -2198 & 1695 & 5543 & 153 \\
\hline $\mathrm{H}(76 \mathrm{C})$ & -3429 & 2309 & 5488 & 153 \\
\hline $\mathrm{H}(78)$ & 2169 & 5544 & 933 & 132 \\
\hline $\mathrm{H}(79)$ & 652 & 5356 & -106 & 202 \\
\hline $\mathrm{H}(80)$ & -411 & 7109 & 56 & 133 \\
\hline $\mathrm{H}(81)$ & 128 & 8832 & 1100 & 122 \\
\hline $\mathrm{H}(82)$ & 1851 & 9033 & 2249 & 145 \\
\hline $\mathrm{H}(83 \mathrm{~A})$ & 3761 & 6612 & 2180 & 354 \\
\hline $\mathrm{H}(83 \mathrm{~B})$ & 3085 & 7422 & 2889 & 354 \\
\hline $\mathrm{H}(83 \mathrm{C})$ & 3797 & 8029 & 2368 & 354 \\
\hline $\mathrm{H}(85)$ & 2065 & 4285 & 2189 & 147 \\
\hline $\mathrm{H}(86)$ & 1245 & 2468 & 1149 & 162 \\
\hline $\mathrm{H}(87)$ & 2051 & 802 & 1370 & 146 \\
\hline $\mathrm{H}(88)$ & 3614 & 813 & 2440 & 136 \\
\hline H(89) & 4342 & 2506 & 3497 & 127 \\
\hline $\mathrm{H}(90 \mathrm{~A})$ & 4040 & 4817 & 4120 & 320 \\
\hline $\mathrm{H}(90 \mathrm{~B})$ & 3546 & 5411 & 3379 & 320 \\
\hline $\mathrm{H}(90 \mathrm{C})$ & 4778 & 4798 & 3387 & 320 \\
\hline $\mathrm{H}(1)$ & 6376 & 8644 & 8366 & 56 \\
\hline $\mathrm{H}(2)$ & 8273 & 6680 & 5492 & 60 \\
\hline $\mathrm{H}(5)$ & 4147 & 3555 & 8370 & 60 \\
\hline $\mathrm{H}(6 \mathrm{~A})$ & 670 & 1645 & 5565 & 58 \\
\hline
\end{tabular}


Table S16. Crystal data and structure refinement for $\mathbf{8}$.

Identification code

Empirical formula

Formula weight

Temperature

Wavelength

Crystal system

Space group

Unit cell dimensions

Volume

Z

Density (calculated)

Absorption coefficient

$\mathrm{F}(000)$

Crystal size

Theta range for data collection

Index ranges

Reflections collected

Independent reflections

Completeness to theta $=28.92^{\circ}$

Absorption correction

Max. and min. transmission

Refinement method

Data / restraints / parameters

Goodness-of-fit on $\mathrm{F}^{2}$

Final R indices [I $>2$ sigma(I)]

$\mathrm{R}$ indices (all data)

Absolute structure parameter

Largest diff. peak and hole
8 (amhex1, $\left(\mathrm{La}_{2}\left(\mathrm{BINOL}-\mathrm{POPh}_{2}\right)_{3}\right.$.6tolunene after Platon Squeeze)

C180 H150 La2 O12 P6

2968.64

293(2) K

$0.71073 \AA$

Hexagonal

P6(3)

$\mathrm{a}=31.7444(16) \AA \quad \alpha=90^{\circ}$

$\mathrm{b}=31.7444(16) \AA \quad \beta=90^{\circ}$

$\mathrm{c}=25.393(3) \AA \quad \gamma=120^{\circ}$

22160(3) $\AA^{3}$

6

$1.335 \mathrm{~g} / \mathrm{cm}^{3}$

$0.699 \mathrm{~mm}^{-1}$

9180

$0.45 \times 0.06 \times 0.05 \mathrm{~mm}^{3}$

0.74 to $28.92^{\circ}$

$-40 \leq \mathrm{h} \leq 42,-42 \leq \mathrm{k} \leq 40,-34 \leq \mathrm{l} \leq 34$

189306

36274 [R(int) $=0.2384]$

$95.1 \%$

Semi-empirical from equivalents

0.9659 and 0.7439

Full-matrix least-squares on $\mathrm{F}^{2}$

36274 / 19 / 1423

0.700

$\mathrm{R} 1=0.0586, \mathrm{wR} 2=0.1193$

$\mathrm{R} 1=0.1699, \mathrm{wR} 2=0.1386$

$-0.004(11)$

0.756 and -0.442 e. $\AA^{-3}$ 
Table S17. Atomic coordinates $\left(\times 10^{4}\right)$ and equivalent isotropic displacement parameters $\left(\AA^{2} \times\right.$ $10^{3}$ ) for 8 . $U(e q)$ is defined as one third of the trace of the orthogonalized $U^{i j}$ tensor.

\begin{tabular}{|c|c|c|c|c|}
\hline & $\mathrm{x}$ & $\mathrm{y}$ & $\mathrm{z}$ & $\mathrm{U}(\mathrm{eq})$ \\
\hline $\mathrm{C}(1)$ & $-902(3)$ & $6265(3)$ & 4841(3) & $34(2)$ \\
\hline $\mathrm{C}(2)$ & $-461(3)$ & 6654(3) & 4990(3) & $37(2)$ \\
\hline C(3) & $-312(3)$ & 7131(3) & 4754(3) & $38(2)$ \\
\hline C(4) & $-570(3)$ & 7212(3) & $4415(3)$ & $44(2)$ \\
\hline$C(5)$ & $-1018(3)$ & 6813(3) & $4237(4)$ & $49(2)$ \\
\hline C(6) & $-1313(4)$ & $6865(4)$ & $3851(4)$ & $60(3)$ \\
\hline C(7) & $-1740(4)$ & $6507(4)$ & 3653(4) & $62(3)$ \\
\hline $\mathrm{C}(8)$ & $-1871(4)$ & $6046(4)$ & $3897(4)$ & 71(3) \\
\hline C(9) & $-1602(3)$ & 5967(3) & $4265(4)$ & $40(2)$ \\
\hline$C(10)$ & $-1178(3)$ & 6329(3) & $4449(3)$ & $35(2)$ \\
\hline$C(11)$ & $-1080(3)$ & 5794(3) & 5058(3) & $35(2)$ \\
\hline$C(12)$ & $-895(3)$ & 5495(3) & 4927(3) & $42(2)$ \\
\hline C(13) & $-1096(3)$ & 5016(3) & 5128(3) & $44(2)$ \\
\hline$C(14)$ & $-1522(4)$ & $4830(4)$ & 5479(3) & $76(4)$ \\
\hline$C(15)$ & $-1696(4)$ & 5133(4) & 5622(3) & $55(3)$ \\
\hline$C(16)$ & $-2078(4)$ & $4946(5)$ & $6014(3)$ & $77(4)$ \\
\hline$C(17)$ & $-2230(3)$ & $5255(5)$ & 6182(3) & $69(3)$ \\
\hline C(18) & $-2046(4)$ & $5729(4)$ & $5975(4)$ & 71(3) \\
\hline C(19) & $-1663(3)$ & $5914(4)$ & 5623(3) & $58(3)$ \\
\hline$C(20)$ & $-1467(3)$ & $5609(4)$ & 5432(3) & $44(2)$ \\
\hline C(21) & 180(3) & 7550(3) & 4931(3) & 51(3) \\
\hline$C(22)$ & $-249(4)$ & $7565(4)$ & 5983(4) & 64(3) \\
\hline C(23) & $-364(5)$ & 7311(4) & $6414(5)$ & 133(6) \\
\hline$C(24)$ & $-736(7)$ & $7140(7)$ & $6720(9)$ & $266(17)$ \\
\hline$C(25)$ & $-1012(7)$ & $7271(6)$ & 6483(8) & 191(12) \\
\hline$C(26)$ & $-979(4)$ & 7553(5) & $6056(6)$ & 148(9) \\
\hline $\mathrm{C}(27)$ & $-540(3)$ & $7710(4)$ & 5788(5) & $96(4)$ \\
\hline C(28) & $687(3)$ & 8354(3) & 5683(4) & $58(3)$ \\
\hline C(29) & $696(5)$ & $8575(4)$ & $6144(5)$ & $134(6)$ \\
\hline$C(30)$ & 1022(5) & $9066(5)$ & $6176(6)$ & $146(7)$ \\
\hline C(31) & $1310(5)$ & $9326(4)$ & $5760(7)$ & 137(6) \\
\hline C(32) & 1307(5) & $9116(4)$ & $5309(6)$ & $141(7)$ \\
\hline C(33) & $1004(5)$ & $8606(4)$ & 5281(5) & $116(5)$ \\
\hline C(34) & $-904(3)$ & 4677(3) & 4982(3) & $45(2)$ \\
\hline C(35) & $-959(3)$ & 3932(3) & $4320(3)$ & $33(2)$ \\
\hline C(36) & $-845(4)$ & $3718(4)$ & 4737(3) & $82(4)$ \\
\hline C(37) & $-877(4)$ & $3244(4)$ & $4720(4)$ & 61(3) \\
\hline C(38) & $-998(3)$ & 3018(3) & $4263(4)$ & $67(3)$ \\
\hline C(39) & $-1116(4)$ & $3225(4)$ & $3810(3)$ & $56(3)$ \\
\hline$C(40)$ & $-1107(3)$ & 3648(3) & $3840(3)$ & 49(3) \\
\hline
\end{tabular}




\begin{tabular}{|c|c|c|c|c|}
\hline $\mathrm{C}(41)$ & $-1395(3)$ & 4494(3) & 4008(3) & $48(2)$ \\
\hline$C(42)$ & $-1869(4)$ & $4064(4)$ & $4050(4)$ & $58(3)$ \\
\hline C(43) & $-2260(4)$ & $4023(4)$ & $3782(5)$ & $84(4)$ \\
\hline C(44) & $-2207(4)$ & $4389(5)$ & $3435(5)$ & $90(4)$ \\
\hline C(45) & $-1736(4)$ & $4801(4)$ & $3371(4)$ & 73(3) \\
\hline C(46) & $-1341(4)$ & $4859(4)$ & 3657(3) & $52(3)$ \\
\hline C(47) & 1603(3) & 7255(3) & $4448(3)$ & $26(2)$ \\
\hline C(48) & 1519(3) & 7047(3) & 4952(3) & $31(2)$ \\
\hline C(49) & 1847(3) & 6931(3) & 5194(3) & $37(2)$ \\
\hline C(50) & 2271(3) & 7031(3) & 4952(3) & $42(2)$ \\
\hline C(51) & 2351(3) & $7220(3)$ & 4421(3) & $38(2)$ \\
\hline C(52) & 2767(3) & $7270(3)$ & $4141(4)$ & $45(2)$ \\
\hline $\mathrm{C}(53)$ & 2836(3) & $7420(3)$ & $3645(4)$ & $44(2)$ \\
\hline C(54) & 2505(3) & 7518(3) & $3385(4)$ & $57(3)$ \\
\hline C(55) & 2108(3) & $7470(3)$ & $3639(3)$ & $39(2)$ \\
\hline C(56) & 2004(3) & $7319(2)$ & $4184(4)$ & $30(2)$ \\
\hline C(57) & 1269(3) & 7392(3) & 4212(3) & $35(2)$ \\
\hline C(58) & $820(3)$ & 7052(3) & $4060(3)$ & $36(2)$ \\
\hline C(59) & 492(3) & 7158(3) & $3777(3)$ & $36(2)$ \\
\hline C(60) & $604(4)$ & 7617(3) & $3684(3)$ & $47(2)$ \\
\hline C(61) & 1106(3) & 8014(3) & 3862(3) & $50(3)$ \\
\hline $\mathrm{C}(62)$ & $1236(4)$ & 8493(3) & $3762(3)$ & $56(3)$ \\
\hline C(63) & $1680(4)$ & $8817(4)$ & $3920(4)$ & 61(3) \\
\hline C(64) & 1961(3) & $8714(3)$ & $4209(4)$ & $53(2)$ \\
\hline C(65) & 1844(3) & $8256(3)$ & 4311(3) & $30(2)$ \\
\hline C(66) & 1416(3) & 7884(3) & 4133(3) & $37(2)$ \\
\hline C(67) & 1731(3) & 6738(3) & 5745(3) & $48(2)$ \\
\hline C(68) & 2035(3) & 7712(3) & 6029(3) & $43(2)$ \\
\hline C(69) & 2503(3) & 7914(3) & 5888(3) & $39(2)$ \\
\hline C(70) & $2753(4)$ & $8355(4)$ & 5685(3) & $55(3)$ \\
\hline $\mathrm{C}(71)$ & 2557(3) & 8641(3) & 5631(3) & $46(3)$ \\
\hline C(72) & 2051(3) & 8437(3) & $5778(4)$ & $52(2)$ \\
\hline C(73) & $1804(4)$ & 7988(3) & 5965(3) & $47(2)$ \\
\hline C(74) & 1958(3) & 7055(3) & 6835(3) & $39(2)$ \\
\hline C(75) & $2069(4)$ & $7368(4)$ & $7244(4)$ & $77(4)$ \\
\hline C(76) & 2195(4) & 7311(4) & 7733(4) & $72(4)$ \\
\hline C(77) & $2235(4)$ & $6875(4)$ & $7829(4)$ & $69(3)$ \\
\hline C(78) & 2123(4) & $6526(4)$ & $7440(4)$ & 81(4) \\
\hline C(79) & 1991(4) & $6623(4)$ & 6953(3) & 81(4) \\
\hline C(80) & $-5(3)$ & $6770(3)$ & $3611(3)$ & $34(2)$ \\
\hline C(81) & 532(3) & 6651(3) & 2761(3) & $36(2)$ \\
\hline C(82) & $868(3)$ & 6501(3) & 2755(3) & $39(2)$ \\
\hline C(83) & 1303(3) & $6761(4)$ & 2488(3) & $52(3)$ \\
\hline C(84) & 1399(4) & $7180(4)$ & 2239(4) & $55(3)$ \\
\hline C(85) & $1112(4)$ & 7361(4) & $2212(4)$ & $65(3)$ \\
\hline
\end{tabular}




\begin{tabular}{|c|c|c|c|c|}
\hline$C(86)$ & $647(3)$ & $7087(3)$ & $2470(3)$ & $57(3)$ \\
\hline C(87) & $-516(3)$ & $6217(3)$ & 2689(3) & $34(2)$ \\
\hline C(88) & $-497(3)$ & 6097(3) & 2162(3) & $50(3)$ \\
\hline C(89) & $-893(5)$ & $5974(4)$ & $1830(4)$ & $95(4)$ \\
\hline $\mathrm{C}(90)$ & $-1292(4)$ & $5966(4)$ & $2038(4)$ & $66(3)$ \\
\hline $\mathrm{C}(91)$ & $-1301(3)$ & $6096(4)$ & 2525(4) & $62(3)$ \\
\hline $\mathrm{C}(92)$ & $-931(3)$ & 6218(3) & 2871(4) & 57(3) \\
\hline C(93) & 271(4) & $5109(4)$ & 5832(3) & $58(3)$ \\
\hline C(94) & $61(4)$ & 5385(3) & 5963(3) & $44(2)$ \\
\hline C(95) & $-378(4)$ & 5185(3) & $6203(3)$ & $49(2)$ \\
\hline C(96) & $-629(4)$ & $4674(4)$ & $6324(3)$ & $65(3)$ \\
\hline C(97) & $-397(5)$ & $4418(4)$ & $6170(3)$ & $66(4)$ \\
\hline C(98) & $-674(5)$ & $3896(4)$ & $6275(3)$ & $91(4)$ \\
\hline C(99) & $-467(5)$ & $3616(4)$ & $6092(4)$ & 71(3) \\
\hline$C(100)$ & $-22(4)$ & $3831(4)$ & $5836(4)$ & $64(3)$ \\
\hline$C(101)$ & 192(4) & $4311(4)$ & $5793(4)$ & $63(3)$ \\
\hline C(102) & $35(4)$ & 4614(3) & 5921(3) & $43(3)$ \\
\hline C(103) & $818(4)$ & 5364(3) & 5661(3) & 49(3) \\
\hline C(104) & $932(3)$ & $5549(3)$ & 5128(3) & $41(2)$ \\
\hline$C(105)$ & $1420(3)$ & 5738(3) & 4937(3) & $40(2)$ \\
\hline$C(106)$ & 1759(4) & 5734(3) & 5246(3) & $57(3)$ \\
\hline$C(107)$ & 1631(3) & 5559(3) & $5792(4)$ & 53(3) \\
\hline C(108) & $2016(4)$ & $5609(4)$ & $6123(4)$ & $71(3)$ \\
\hline C(109) & $1920(6)$ & $5456(6)$ & $6636(4)$ & 124(7) \\
\hline$C(110)$ & 1425(6) & $5252(4)$ & $6851(4)$ & 108(5) \\
\hline $\mathrm{C}(111)$ & 1082(4) & 5231(3) & $6501(4)$ & $70(3)$ \\
\hline $\mathrm{C}(112)$ & $1189(4)$ & 5395(3) & $5972(3)$ & $45(3)$ \\
\hline C(113) & $-609(3)$ & 5488(3) & $6357(3)$ & $55(3)$ \\
\hline$C(114)$ & $19(5)$ & $5727(4)$ & 7264(3) & $61(3)$ \\
\hline $\mathrm{C}(115)$ & $-216(5)$ & $5269(4)$ & $7496(4)$ & $70(3)$ \\
\hline$C(116)$ & $36(5)$ & 5075(5) & $7740(4)$ & $72(3)$ \\
\hline $\mathrm{C}(117)$ & 523(5) & $5362(5)$ & $7752(4)$ & $89(4)$ \\
\hline$C(118)$ & 717(5) & $5800(5)$ & $7539(4)$ & $93(4)$ \\
\hline C(119) & 527(4) & $6013(4)$ & $7280(3)$ & $56(3)$ \\
\hline $\mathrm{C}(120)$ & $-755(3)$ & $5940(3)$ & 7251(3) & $46(2)$ \\
\hline $\mathrm{C}(121)$ & $-699(4)$ & $6050(4)$ & 7761(3) & $67(3)$ \\
\hline $\mathrm{C}(122)$ & $-1001(5)$ & $6138(5)$ & $8053(5)$ & $110(5)$ \\
\hline$C(123)$ & $-1385(5)$ & $6123(4)$ & 7833(5) & $81(4)$ \\
\hline$C(124)$ & $-1493(4)$ & $5992(4)$ & 7297(5) & $95(4)$ \\
\hline$C(125)$ & $-1157(4)$ & $5920(3)$ & $7019(4)$ & $70(3)$ \\
\hline$C(126)$ & 1507(3) & 5917(3) & 4378(3) & $45(2)$ \\
\hline $\mathrm{C}(127)$ & 977(3) & 4928(3) & 4020(3) & $31(2)$ \\
\hline C(128) & $478(3)$ & $4587(4)$ & 3974(3) & $47(2)$ \\
\hline C(129) & 298(4) & 4120(3) & $4147(5)$ & $70(3)$ \\
\hline $\mathrm{C}(130)$ & $638(4)$ & 4025(3) & $4372(3)$ & $58(3)$ \\
\hline
\end{tabular}




\begin{tabular}{|c|c|c|c|c|}
\hline $\mathrm{C}(131)$ & 1095(3) & $4327(4)$ & $4419(3)$ & $47(3)$ \\
\hline $\mathrm{C}(132)$ & 1254(3) & 4775(3) & $4250(4)$ & $43(2)$ \\
\hline C(133) & 1586(3) & $5677(3)$ & $3306(3)$ & $32(2)$ \\
\hline$C(134)$ & 1552(3) & $5329(3)$ & $2968(4)$ & $48(3)$ \\
\hline$C(135)$ & $1839(4)$ & $5437(4)$ & 2521(3) & $67(3)$ \\
\hline$C(136)$ & 2222(3) & $5940(4)$ & $2425(4)$ & $62(3)$ \\
\hline$C(137)$ & $2286(5)$ & $6313(4)$ & $2748(4)$ & $84(4)$ \\
\hline C(138) & $1949(4)$ & $6159(4)$ & $3201(4)$ & 59(3) \\
\hline $\mathrm{La}(1)$ & 469(1) & 6664(1) & 5792(1) & $36(1)$ \\
\hline $\mathrm{La}(2)$ & $178(1)$ & 5731(1) & $4208(1)$ & $33(1)$ \\
\hline $\mathrm{O}(1)$ & $-172(2)$ & $6618(2)$ & $5323(2)$ & $44(2)$ \\
\hline $\mathrm{O}(2)$ & $-508(2)$ & $5644(2)$ & $4615(2)$ & $42(2)$ \\
\hline $\mathrm{O}(3)$ & $557(2)$ & $7482(2)$ & $5916(2)$ & $43(2)$ \\
\hline $\mathrm{O}(4)$ & $-421(2)$ & $4869(2)$ & $4040(2)$ & $46(2)$ \\
\hline $\mathrm{O}(5)$ & $1129(2)$ & $6957(2)$ & $5229(2)$ & $42(2)$ \\
\hline $\mathrm{O}(6)$ & $646(2)$ & $6554(2)$ & $4134(2)$ & $40(1)$ \\
\hline $\mathrm{O}(7)$ & $1180(2)$ & 6982(2) & $6401(2)$ & $48(2)$ \\
\hline $\mathrm{O}(8)$ & $-89(2)$ & $5867(2)$ & $3340(2)$ & $39(1)$ \\
\hline $\mathrm{O}(9)$ & $286(2)$ & $5874(2)$ & $5866(2)$ & $49(2)$ \\
\hline $\mathrm{O}(10)$ & $584(2)$ & $5525(2)$ & $4805(2)$ & $43(2)$ \\
\hline $\mathrm{O}(11)$ & $25(2)$ & $6432(2)$ & $6638(2)$ & $43(2)$ \\
\hline $\mathrm{O}(12)$ & $767(2)$ & 5619(2) & $3656(2)$ & $37(2)$ \\
\hline $\mathrm{P}(1)$ & $310(1)$ & 7707(1) & $5635(1)$ & $44(1)$ \\
\hline $\mathrm{P}(2)$ & $-885(1)$ & $4525(1)$ & 4323(1) & $39(1)$ \\
\hline $\mathrm{P}(3)$ & 1682(1) & 7103(1) & $6253(1)$ & $40(1)$ \\
\hline $\mathrm{P}(4)$ & $-18(1)$ & $6342(1)$ & 3123(1) & $34(1)$ \\
\hline$P(5)$ & $-311(1)$ & 5931(1) & 6852(1) & $45(1)$ \\
\hline$P(6)$ & $1170(1)$ & $5541(1)$ & $3835(1)$ & $37(1)$ \\
\hline
\end{tabular}


Table S18. Bond lengths $[\AA]$ and angles [ $\left.{ }^{\circ}\right]$ for 8 .

\begin{tabular}{|c|c|c|c|}
\hline $\mathrm{C}(1)-\mathrm{C}(2)$ & $1.378(11)$ & $\mathrm{C}(35)-\mathrm{C}(36)$ & $1.398(11)$ \\
\hline $\mathrm{C}(1)-\mathrm{C}(10)$ & $1.406(11)$ & C(35)-C(40) & $1.447(10)$ \\
\hline $\mathrm{C}(1)-\mathrm{C}(11)$ & $1.421(10)$ & $\mathrm{C}(35)-\mathrm{P}(2)$ & $1.778(8)$ \\
\hline $\mathrm{C}(2)-\mathrm{O}(1)$ & $1.293(9)$ & $\mathrm{C}(36)-\mathrm{C}(37)$ & $1.455(13)$ \\
\hline $\mathrm{C}(2)-\mathrm{C}(3)$ & $1.471(11)$ & $\mathrm{C}(37)-\mathrm{C}(38)$ & $1.316(12)$ \\
\hline C(3)-C(4) & $1.300(10)$ & C(38)-C(39) & $1.466(12)$ \\
\hline$C(3)-C(21)$ & $1.526(11)$ & $\mathrm{C}(39)-\mathrm{C}(40)$ & $1.330(11)$ \\
\hline$C(4)-C(5)$ & $1.426(11)$ & $\mathrm{C}(41)-\mathrm{C}(46)$ & $1.403(11)$ \\
\hline$C(5)-C(6)$ & $1.422(12)$ & $\mathrm{C}(41)-\mathrm{C}(42)$ & $1.443(12)$ \\
\hline$C(5)-C(10)$ & $1.457(11)$ & $\mathrm{C}(41)-\mathrm{P}(2)$ & $1.763(9)$ \\
\hline$C(6)-C(7)$ & $1.356(12)$ & $\mathrm{C}(42)-\mathrm{C}(43)$ & $1.364(13)$ \\
\hline $\mathrm{C}(7)-\mathrm{C}(8)$ & $1.444(13)$ & $C(43)-C(44)$ & $1.400(15)$ \\
\hline $\mathrm{C}(8)-\mathrm{C}(9)$ & $1.372(12)$ & $\mathrm{C}(44)-\mathrm{C}(45)$ & $1.419(14)$ \\
\hline C(9)-C(10) & $1.345(11)$ & $C(45)-C(46)$ & $1.382(12)$ \\
\hline $\mathrm{C}(11)-\mathrm{C}(12)$ & $1.382(11)$ & $\mathrm{C}(47)-\mathrm{C}(48)$ & $1.402(10)$ \\
\hline $\mathrm{C}(11)-\mathrm{C}(20)$ & $1.426(10)$ & $C(47)-C(56)$ & $1.364(9)$ \\
\hline $\mathrm{C}(12)-\mathrm{O}(2)$ & $1.336(9)$ & $\mathrm{C}(47)-\mathrm{C}(57)$ & $1.462(10)$ \\
\hline $\mathrm{C}(12)-\mathrm{C}(13)$ & $1.419(11)$ & $\mathrm{C}(48)-\mathrm{O}(5)$ & $1.326(8)$ \\
\hline $\mathrm{C}(13)-\mathrm{C}(14)$ & $1.473(12)$ & $\mathrm{C}(48)-\mathrm{C}(49)$ & $1.408(10)$ \\
\hline $\mathrm{C}(13)-\mathrm{C}(34)$ & $1.523(11)$ & $\mathrm{C}(49)-\mathrm{C}(50)$ & $1.364(11)$ \\
\hline $\mathrm{C}(14)-\mathrm{C}(15)$ & $1.374(13)$ & $\mathrm{C}(49)-\mathrm{C}(67)$ & $1.498(11)$ \\
\hline $\mathrm{C}(15)-\mathrm{C}(20)$ & $1.395(12)$ & $\mathrm{C}(50)-\mathrm{C}(51)$ & $1.447(10)$ \\
\hline $\mathrm{C}(15)-\mathrm{C}(16)$ & $1.446(12)$ & $\mathrm{C}(51)-\mathrm{C}(56)$ & $1.419(10)$ \\
\hline $\mathrm{C}(16)-\mathrm{C}(17)$ & $1.359(14)$ & $\mathrm{C}(51)-\mathrm{C}(52)$ & $1.436(11)$ \\
\hline $\mathrm{C}(17)-\mathrm{C}(18)$ & $1.416(13)$ & $C(52)-C(53)$ & $1.326(11)$ \\
\hline $\mathrm{C}(18)-\mathrm{C}(19)$ & $1.380(11)$ & $\mathrm{C}(53)-\mathrm{C}(54)$ & $1.402(12)$ \\
\hline $\mathrm{C}(19)-\mathrm{C}(20)$ & $1.473(12)$ & $\mathrm{C}(54)-\mathrm{C}(55)$ & $1.354(11)$ \\
\hline $\mathrm{C}(21)-\mathrm{P}(1)$ & $1.848(8)$ & $\mathrm{C}(55)-\mathrm{C}(56)$ & $1.446(11)$ \\
\hline $\mathrm{C}(22)-\mathrm{C}(23)$ & $1.300(12)$ & $\mathrm{C}(57)-\mathrm{C}(58)$ & $1.344(10)$ \\
\hline $\mathrm{C}(22)-\mathrm{C}(27)$ & $1.316(11)$ & $\mathrm{C}(57)-\mathrm{C}(66)$ & $1.402(10)$ \\
\hline $\mathrm{C}(22)-\mathrm{P}(1)$ & $1.826(9)$ & $\mathrm{C}(58)-\mathrm{O}(6)$ & $1.403(9)$ \\
\hline $\mathrm{C}(23)-\mathrm{C}(24)$ & $1.285(15)$ & C(58)-C(59) & $1.436(10)$ \\
\hline $\mathrm{C}(24)-\mathrm{C}(25)$ & $1.291(16)$ & $\mathrm{C}(59)-\mathrm{C}(60)$ & $1.337(10)$ \\
\hline$C(25)-C(26)$ & $1.375(15)$ & $\mathrm{C}(59)-\mathrm{C}(80)$ & $1.498(10)$ \\
\hline $\mathrm{C}(26)-\mathrm{C}(27)$ & $1.400(12)$ & $\mathrm{C}(60)-\mathrm{C}(61)$ & $1.523(12)$ \\
\hline $\mathrm{C}(28)-\mathrm{C}(29)$ & $1.360(11)$ & $\mathrm{C}(61)-\mathrm{C}(62)$ & $1.386(12)$ \\
\hline $\mathrm{C}(28)-\mathrm{C}(33)$ & $1.373(11)$ & $\mathrm{C}(61)-\mathrm{C}(66)$ & $1.420(11)$ \\
\hline $\mathrm{C}(28)-\mathrm{P}(1)$ & 1.791(10) & $\mathrm{C}(62)-\mathrm{C}(63)$ & $1.324(12)$ \\
\hline $\mathrm{C}(29)-\mathrm{C}(30)$ & $1.377(13)$ & $\mathrm{C}(63)-\mathrm{C}(64)$ & $1.316(11)$ \\
\hline $\mathrm{C}(30)-\mathrm{C}(31)$ & $1.368(13)$ & $\mathrm{C}(64)-\mathrm{C}(65)$ & 1.333(10) \\
\hline $\mathrm{C}(31)-\mathrm{C}(32)$ & $1.324(13)$ & $C(65)-C(66)$ & 1.357(10) \\
\hline $\mathrm{C}(32)-\mathrm{C}(33)$ & $1.413(12)$ & $\mathrm{C}(67)-\mathrm{P}(3)$ & 1.791(8) \\
\hline $\mathrm{C}(34)-\mathrm{P}(2)$ & $1.752(8)$ & $\mathrm{C}(68)-\mathrm{C}(73)$ & $1.406(11)$ \\
\hline
\end{tabular}




\begin{tabular}{|c|c|c|c|}
\hline $\mathrm{C}(68)-\mathrm{C}(69)$ & $1.339(10)$ & $C(104)-C(105)$ & $1.437(11)$ \\
\hline C(68)-P(3) & $1.774(9)$ & C(105)-C(106) & $1.339(11)$ \\
\hline C(69)-C(70) & $1.321(11)$ & $C(105)-C(126)$ & $1.504(10)$ \\
\hline $\mathrm{C}(70)-\mathrm{C}(71)$ & $1.340(11)$ & $C(106)-C(107)$ & $1.472(13)$ \\
\hline $\mathrm{C}(71)-\mathrm{C}(72)$ & $1.448(11)$ & $\mathrm{C}(107)-\mathrm{C}(112)$ & $1.310(12)$ \\
\hline C(72)-C(73) & $1.325(11)$ & C(107)-C(108) & $1.426(12)$ \\
\hline $\mathrm{C}(74)-\mathrm{C}(75)$ & $1.355(11)$ & C(108)-C(109) & $1.369(15)$ \\
\hline $\mathrm{C}(74)-\mathrm{C}(79)$ & $1.460(12)$ & $\mathrm{C}(109)-\mathrm{C}(110)$ & $1.472(18)$ \\
\hline $\mathrm{C}(74)-\mathrm{P}(3)$ & $1.763(8)$ & $\mathrm{C}(110)-\mathrm{C}(111)$ & $1.382(14)$ \\
\hline $\mathrm{C}(75)-\mathrm{C}(76)$ & $1.346(12)$ & $\mathrm{C}(111)-\mathrm{C}(112)$ & $1.419(12)$ \\
\hline $\mathrm{C}(76)-\mathrm{C}(77)$ & $1.473(13)$ & $\mathrm{C}(113)-\mathrm{P}(5)$ & $1.768(8)$ \\
\hline $\mathrm{C}(77)-\mathrm{C}(78)$ & $1.390(12)$ & $\mathrm{C}(114)-\mathrm{C}(119)$ & $1.402(13)$ \\
\hline C(78)-C(79) & $1.386(12)$ & $\mathrm{C}(114)-\mathrm{C}(115)$ & $1.389(13)$ \\
\hline $\mathrm{C}(80)-\mathrm{P}(4)$ & $1.825(7)$ & $\mathrm{C}(114)-\mathrm{P}(5)$ & $1.814(12)$ \\
\hline $\mathrm{C}(81)-\mathrm{C}(82)$ & $1.368(11)$ & $C(115)-C(116)$ & $1.375(13)$ \\
\hline $\mathrm{C}(81)-\mathrm{C}(86)$ & $1.446(11)$ & $\mathrm{C}(116)-\mathrm{C}(117)$ & $1.346(14)$ \\
\hline $\mathrm{C}(81)-\mathrm{P}(4)$ & $1.773(8)$ & $\mathrm{C}(117)-\mathrm{C}(118)$ & $1.321(15)$ \\
\hline $\mathrm{C}(82)-\mathrm{C}(83)$ & $1.382(11)$ & $\mathrm{C}(118)-\mathrm{C}(119)$ & $1.291(13)$ \\
\hline C(83)-C(84) & $1.363(12)$ & $\mathrm{C}(120)-\mathrm{C}(121)$ & $1.331(11)$ \\
\hline $\mathrm{C}(84)-\mathrm{C}(85)$ & $1.298(12)$ & $C(120)-C(125)$ & $1.378(12)$ \\
\hline $\mathrm{C}(85)-\mathrm{C}(86)$ & $1.442(12)$ & $\mathrm{C}(120)-\mathrm{P}(5)$ & $1.747(9)$ \\
\hline $\mathrm{C}(87)-\mathrm{C}(92)$ & $1.398(11)$ & $\mathrm{C}(121)-\mathrm{C}(122)$ & $1.345(13)$ \\
\hline C(87)-C(88) & $1.400(10)$ & C(122)-C(123) & $1.322(15)$ \\
\hline $\mathrm{C}(87)-\mathrm{P}(4)$ & $1.802(8)$ & $\mathrm{C}(123)-\mathrm{C}(124)$ & $1.414(14)$ \\
\hline C(88)-C(89) & $1.398(11)$ & $\mathrm{C}(124)-\mathrm{C}(125)$ & $1.389(14)$ \\
\hline C(89)-C(90) & $1.361(14)$ & $\mathrm{C}(126)-\mathrm{P}(6)$ & $1.786(7)$ \\
\hline $\mathrm{C}(90)-\mathrm{C}(91)$ & $1.308(11)$ & $\mathrm{C}(127)-\mathrm{C}(132)$ & $1.334(10)$ \\
\hline C(91)-C(92) & $1.357(11)$ & $\mathrm{C}(127)-\mathrm{C}(128)$ & $1.408(10)$ \\
\hline C(93)-C(94) & $1.381(11)$ & $\mathrm{C}(127)-\mathrm{P}(6)$ & $1.787(8)$ \\
\hline $\mathrm{C}(93)-\mathrm{C}(102)$ & $1.379(12)$ & $\mathrm{C}(128)-\mathrm{C}(129)$ & $1.369(11)$ \\
\hline C(93)-C(103) & $1.565(13)$ & C(129)-C(130) & $1.382(13)$ \\
\hline $\mathrm{C}(94)-\mathrm{O}(9)$ & $1.368(10)$ & $\mathrm{C}(130)-\mathrm{C}(131)$ & $1.283(12)$ \\
\hline C(94)-C(95) & $1.352(11)$ & $\mathrm{C}(131)-\mathrm{C}(132)$ & $1.320(11)$ \\
\hline C(95)-C(96) & $1.438(12)$ & C(133)-C(134) & $1.362(10)$ \\
\hline $\mathrm{C}(95)-\mathrm{C}(113)$ & $1.524(12)$ & C(133)-C(138) & $1.405(12)$ \\
\hline $\mathrm{C}(96)-\mathrm{C}(97)$ & $1.396(13)$ & C(133)-P(6) & $1.779(8)$ \\
\hline C(97)-C(102) & $1.347(13)$ & $\mathrm{C}(134)-\mathrm{C}(135)$ & $1.385(11)$ \\
\hline $\mathrm{C}(97)-\mathrm{C}(98)$ & $1.461(13)$ & $\mathrm{C}(135)-\mathrm{C}(136)$ & $1.466(13)$ \\
\hline C(98)-C(99) & $1.422(14)$ & $\mathrm{C}(136)-\mathrm{C}(137)$ & $1.369(13)$ \\
\hline C(99)-C(100) & $1.385(13)$ & C(137)-C(138) & $1.479(13)$ \\
\hline$C(100)-C(101)$ & $1.326(11)$ & $\mathrm{La}(1)-\mathrm{O}(9)$ & $2.282(6)$ \\
\hline$C(101)-C(102)$ & $1.328(12)$ & $\mathrm{La}(1)-\mathrm{O}(1)$ & $2.298(6)$ \\
\hline C(103)-C(112) & $1.380(11)$ & $\mathrm{La}(1)-\mathrm{O}(5)$ & $2.311(6)$ \\
\hline C(103)-C(104) & $1.448(11)$ & $\mathrm{La}(1)-\mathrm{O}(11)$ & $2.472(5)$ \\
\hline $\mathrm{C}(104)-\mathrm{O}(10)$ & $1.347(9)$ & $\mathrm{La}(1)-\mathrm{O}(7)$ & $2.497(6)$ \\
\hline
\end{tabular}




$\begin{array}{lr}\mathrm{La}(1)-\mathrm{O}(3) & 2.489(5) \\ \mathrm{La}(2)-\mathrm{O}(10) & 2.285(6) \\ \mathrm{La}(2)-\mathrm{O}(6) & 2.278(5) \\ \mathrm{La}(2)-\mathrm{O}(2) & 2.299(6) \\ \mathrm{La}(2)-\mathrm{O}(4) & 2.467(6) \\ \mathrm{La}(2)-\mathrm{O}(8) & 2.477(5) \\ \mathrm{La}(2)-\mathrm{O}(12) & 2.499(5) \\ \mathrm{C}(2)-\mathrm{C}(1)-\mathrm{C}(10) & 119.6(8) \\ \mathrm{C}(2)-\mathrm{C}(1)-\mathrm{C}(11) & 121.8(8) \\ \mathrm{C}(10)-\mathrm{C}(1)-\mathrm{C}(11) & 118.6(8) \\ \mathrm{O}(1)-\mathrm{C}(2)-\mathrm{C}(1) & 123.4(8) \\ \mathrm{O}(1)-\mathrm{C}(2)-\mathrm{C}(3) & 118.7(8) \\ \mathrm{C}(1)-\mathrm{C}(2)-\mathrm{C}(3) & 117.9(8) \\ \mathrm{C}(4)-\mathrm{C}(3)-\mathrm{C}(2) & 124.3(8) \\ \mathrm{C}(4)-\mathrm{C}(3)-\mathrm{C}(21) & 119.6(8) \\ \mathrm{C}(2)-\mathrm{C}(3)-\mathrm{C}(21) & 116.1(8) \\ \mathrm{C}(3)-\mathrm{C}(4)-\mathrm{C}(5) & 118.9(9) \\ \mathrm{C}(4)-\mathrm{C}(5)-\mathrm{C}(6) & 122.6(9) \\ \mathrm{C}(4)-\mathrm{C}(5)-\mathrm{C}(10) & 119.0(8) \\ \mathrm{C}(6)-\mathrm{C}(5)-\mathrm{C}(10) & 118.4(8) \\ \mathrm{C}(7)-\mathrm{C}(6)-\mathrm{C}(5) & 126.7(10) \\ \mathrm{C}(6)-\mathrm{C}(7)-\mathrm{C}(8) & 110.5(9) \\ \mathrm{C}(9)-\mathrm{C}(8)-\mathrm{C}(7) & 126.0(10) \\ \mathrm{C}(10)-\mathrm{C}(9)-\mathrm{C}(8) & 121.9(9) \\ \mathrm{C}(9)-\mathrm{C}(10)-\mathrm{C}(1) & 123.5(9) \\ \mathrm{C}(9)-\mathrm{C}(10)-\mathrm{C}(5) & 116.4(8) \\ \mathrm{C}(1)-\mathrm{C}(10)-\mathrm{C}(5) & 120.0(8) \\ \mathrm{C}(12)-\mathrm{C}(11)-\mathrm{C}(20) & 116.8(8) \\ \mathrm{C}(12)-\mathrm{C}(11)-\mathrm{C}(1) & 123.2(8) \\ \mathrm{C}(20)-\mathrm{C}(11)-\mathrm{C}(1) & 120.0(8) \\ \mathrm{O}(2)-\mathrm{C}(12)-\mathrm{C}(13) & 115.8(8) \\ \mathrm{O}(2)-\mathrm{C}(12)-\mathrm{C}(11) & 122.1(8) \\ \mathrm{C}(13)-\mathrm{C}(12)-\mathrm{C}(11) & 122.1(8) \\ \mathrm{C}(12)-\mathrm{C}(13)-\mathrm{C}(14) & 118.4(9) \\ \mathrm{C}(12)-\mathrm{C}(13)-\mathrm{C}(34) & 123.0(8) \\ \mathrm{C}(14)-\mathrm{C}(13)-\mathrm{C}(34) & 118.5(8) \\ \mathrm{C}(15)-\mathrm{C}(14)-\mathrm{C}(13) & 119.4(10) \\ \mathrm{C}(20)-\mathrm{C}(15)-\mathrm{C}(14) & 119.3(9) \\ \mathrm{C}(20)-\mathrm{C}(15)-\mathrm{C}(16) & 123.9(10) \\ \mathrm{C}(14)-\mathrm{C}(15)-\mathrm{C}(16) & 116.4(10) \\ \mathrm{C}(17)-\mathrm{C}(16)-\mathrm{C}(15) & 116.7(11) \\ \mathrm{C}(16)-\mathrm{C}(17)-\mathrm{C}(18) & 122.9(10) \\ \mathrm{C}(17)-\mathrm{C}(18)-\mathrm{C}(19) & 120.0(10) \\ \mathrm{C}(18)-\mathrm{C}(19)-\mathrm{C}(20) & 120.4(10)\end{array}$

$\begin{array}{ll}\mathrm{O}(3)-\mathrm{P}(1) & 1.482(5) \\ \mathrm{O}(4)-\mathrm{P}(2) & 1.507(5) \\ \mathrm{O}(7)-\mathrm{P}(3) & 1.488(6) \\ \mathrm{O}(8)-\mathrm{P}(4) & 1.511(5) \\ \mathrm{O}(11)-\mathrm{P}(5) & 1.504(5) \\ \mathrm{O}(12)-\mathrm{P}(6) & 1.491(5)\end{array}$

$\begin{array}{ll}\mathrm{C}(15)-\mathrm{C}(20)-\mathrm{C}(11) & 123.6(9) \\ \mathrm{C}(15)-\mathrm{C}(20)-\mathrm{C}(19) & 115.8(8) \\ \mathrm{C}(11)-\mathrm{C}(20)-\mathrm{C}(19) & 120.6(9) \\ \mathrm{C}(3)-\mathrm{C}(21)-\mathrm{P}(1) & 120.8(6) \\ \mathrm{C}(23)-\mathrm{C}(22)-\mathrm{C}(27) & 120.5(11) \\ \mathrm{C}(23)-\mathrm{C}(22)-\mathrm{P}(1) & 119.7(9) \\ \mathrm{C}(27)-\mathrm{C}(22)-\mathrm{P}(1) & 119.7(9) \\ \mathrm{C}(24)-\mathrm{C}(23)-\mathrm{C}(22) & 133.1(16) \\ \mathrm{C}(23)-\mathrm{C}(24)-\mathrm{C}(25) & 102(2) \\ \mathrm{C}(24)-\mathrm{C}(25)-\mathrm{C}(26) & 137(2) \\ \mathrm{C}(25)-\mathrm{C}(26)-\mathrm{C}(27) & 110.7(16) \\ \mathrm{C}(22)-\mathrm{C}(27)-\mathrm{C}(26) & 116.0(13) \\ \mathrm{C}(29)-\mathrm{C}(28)-\mathrm{C}(33) & 121.4(10) \\ \mathrm{C}(29)-\mathrm{C}(28)-\mathrm{P}(1) & 118.6(9) \\ \mathrm{C}(33)-\mathrm{C}(28)-\mathrm{P}(1) & 119.5(8) \\ \mathrm{C}(28)-\mathrm{C}(29)-\mathrm{C}(30) & 116.3(12) \\ \mathrm{C}(31)-\mathrm{C}(30)-\mathrm{C}(29) & 122.4(13) \\ \mathrm{C}(32)-\mathrm{C}(31)-\mathrm{C}(30) & 121.9(12) \\ \mathrm{C}(31)-\mathrm{C}(32)-\mathrm{C}(33) & 116.9(13) \\ \mathrm{C}(28)-\mathrm{C}(33)-\mathrm{C}(32) & 120.6(12) \\ \mathrm{C}(13)-\mathrm{C}(34)-\mathrm{P}(2) & 120.6(6) \\ \mathrm{C}(36)-\mathrm{C}(35)-\mathrm{C}(40) & 114.7(8) \\ \mathrm{C}(36)-\mathrm{C}(35)-\mathrm{P}(2) & 125.7(7) \\ \mathrm{C}(40)-\mathrm{C}(35)-\mathrm{P}(2) & 119.5(6) \\ \mathrm{C}(35)-\mathrm{C}(36)-\mathrm{C}(37) & 125.3(8) \\ \mathrm{C}(38)-\mathrm{C}(37)-\mathrm{C}(36) & 116.1(9) \\ \mathrm{C}(37)-\mathrm{C}(38)-\mathrm{C}(39) & 121.1(9) \\ \mathrm{C}(40)-\mathrm{C}(39)-\mathrm{C}(38) & 121.8(9) \\ \mathrm{C}(39)-\mathrm{C}(40)-\mathrm{C}(35) & 120.9(8) \\ \mathrm{C}(46)-\mathrm{C}(41)-\mathrm{C}(42) & 117.9(9) \\ \mathrm{C}(46)-\mathrm{C}(41)-\mathrm{P}(2) & 120.7(8) \\ \mathrm{C}(42)-\mathrm{C}(41)-\mathrm{P}(2) & 120.8(7) \\ \mathrm{C}(43)-\mathrm{C}(42)-\mathrm{C}(41) & 121.4(10) \\ \mathrm{C}(42)-\mathrm{C}(43)-\mathrm{C}(44) & 120.6(11) \\ \mathrm{C}(43)-\mathrm{C}(44)-\mathrm{C}(45) & 118.2(11) \\ \mathrm{C}(46)-\mathrm{C}(45)-\mathrm{C}(44) & 121.9(11) \\ \mathrm{C}(45)-\mathrm{C}(46)-\mathrm{C}(41) & 119.8(10)\end{array}$




\begin{tabular}{|c|c|}
\hline$C(48)-C(47)-C(56)$ & $117.6(7)$ \\
\hline $\mathrm{C}(48)-\mathrm{C}(47)-\mathrm{C}(57)$ & $121.3(7)$ \\
\hline$C(56)-C(47)-C(57)$ & $121.0(7)$ \\
\hline $\mathrm{O}(5)-\mathrm{C}(48)-\mathrm{C}(47)$ & $121.5(7)$ \\
\hline $\mathrm{O}(5)-\mathrm{C}(48)-\mathrm{C}(49)$ & $116.6(7)$ \\
\hline$C(47)-C(48)-C(49)$ & $121.9(7)$ \\
\hline$C(50)-C(49)-C(48)$ & $121.0(8)$ \\
\hline$C(50)-C(49)-C(67)$ & $122.0(8)$ \\
\hline$C(48)-C(49)-C(67)$ & 116.8(8) \\
\hline$C(49)-C(50)-C(51)$ & 117.8(8) \\
\hline$C(56)-C(51)-C(50)$ & $119.4(8)$ \\
\hline $\mathrm{C}(56)-\mathrm{C}(51)-\mathrm{C}(52)$ & $122.5(7)$ \\
\hline$C(50)-C(51)-C(52)$ & $117.9(8)$ \\
\hline$C(53)-C(52)-C(51)$ & 119.1(8) \\
\hline$C(52)-C(53)-C(54)$ & $121.3(9)$ \\
\hline$C(55)-C(54)-C(53)$ & $120.5(8)$ \\
\hline$C(54)-C(55)-C(56)$ & $122.5(8)$ \\
\hline$C(47)-C(56)-C(51)$ & $121.9(8)$ \\
\hline$C(47)-C(56)-C(55)$ & $124.1(7)$ \\
\hline $\mathrm{C}(51)-\mathrm{C}(56)-\mathrm{C}(55)$ & $113.9(7)$ \\
\hline $\mathrm{C}(58)-\mathrm{C}(57)-\mathrm{C}(66)$ & 118.8(7) \\
\hline $\mathrm{C}(58)-\mathrm{C}(57)-\mathrm{C}(47)$ & $120.9(7)$ \\
\hline$C(66)-C(57)-C(47)$ & $120.2(7)$ \\
\hline $\mathrm{C}(57)-\mathrm{C}(58)-\mathrm{O}(6)$ & $122.0(7)$ \\
\hline C(57)-C(58)-C(59) & $123.9(8)$ \\
\hline $\mathrm{O}(6)-\mathrm{C}(58)-\mathrm{C}(59)$ & $114.0(8)$ \\
\hline $\mathrm{C}(60)-\mathrm{C}(59)-\mathrm{C}(58)$ & $120.9(8)$ \\
\hline C(60)-C(59)-C(80) & $116.4(7)$ \\
\hline C(58)-C(59)-C(80) & $122.5(8)$ \\
\hline$C(59)-C(60)-C(61)$ & $116.5(8)$ \\
\hline$C(62)-C(61)-C(66)$ & $122.5(9)$ \\
\hline$C(62)-C(61)-C(60)$ & $117.8(9)$ \\
\hline$C(66)-C(61)-C(60)$ & $119.6(8)$ \\
\hline $\mathrm{C}(63)-\mathrm{C}(62)-\mathrm{C}(61)$ & $114.4(10)$ \\
\hline$C(62)-C(63)-C(64)$ & $124.5(10)$ \\
\hline$C(65)-C(64)-C(63)$ & $121.6(9)$ \\
\hline $\mathrm{C}(64)-\mathrm{C}(65)-\mathrm{C}(66)$ & 119.7(8) \\
\hline $\mathrm{C}(65)-\mathrm{C}(66)-\mathrm{C}(61)$ & 116.5(8) \\
\hline$C(65)-C(66)-C(57)$ & $123.5(8)$ \\
\hline$C(61)-C(66)-C(57)$ & $119.9(7)$ \\
\hline C(49)-C(67)-P(3) & $119.6(6)$ \\
\hline $\mathrm{C}(73)-\mathrm{C}(68)-\mathrm{C}(69)$ & 118.0(9) \\
\hline $\mathrm{C}(73)-\mathrm{C}(68)-\mathrm{P}(3)$ & $118.3(7)$ \\
\hline $\mathrm{C}(69)-\mathrm{C}(68)-\mathrm{P}(3)$ & $123.6(8)$ \\
\hline $\mathrm{C}(70)-C(69)-C(68)$ & $123.2(9)$ \\
\hline
\end{tabular}

\begin{tabular}{|c|c|}
\hline$C(69)-C(70)-C(71)$ & 121.4(9) \\
\hline$C(70)-C(71)-C(72)$ & 117.3(8) \\
\hline$C(73)-C(72)-C(71)$ & $120.0(8)$ \\
\hline$C(68)-C(73)-C(72)$ & $120.0(9)$ \\
\hline $\mathrm{C}(75)-\mathrm{C}(74)-\mathrm{C}(79)$ & 114.7(8) \\
\hline $\mathrm{C}(75)-\mathrm{C}(74)-\mathrm{P}(3)$ & $122.5(7)$ \\
\hline $\mathrm{C}(79)-\mathrm{C}(74)-\mathrm{P}(3)$ & $121.7(6)$ \\
\hline$C(74)-C(75)-C(76)$ & $126.4(11)$ \\
\hline$C(75)-C(76)-C(77)$ & 116.8(9) \\
\hline$C(78)-C(77)-C(76)$ & 121.5(8) \\
\hline$C(77)-C(78)-C(79)$ & 116.3(10) \\
\hline$C(78)-C(79)-C(74)$ & $124.2(9)$ \\
\hline $\mathrm{C}(59)-\mathrm{C}(80)-\mathrm{P}(4)$ & $115.2(6)$ \\
\hline$C(82)-C(81)-C(86)$ & $117.4(7)$ \\
\hline $\mathrm{C}(82)-\mathrm{C}(81)-\mathrm{P}(4)$ & $122.5(7)$ \\
\hline $\mathrm{C}(86)-\mathrm{C}(81)-\mathrm{P}(4)$ & $120.1(7)$ \\
\hline $\mathrm{C}(83)-\mathrm{C}(82)-\mathrm{C}(81)$ & 121.1(8) \\
\hline $\mathrm{C}(84)-\mathrm{C}(83)-\mathrm{C}(82)$ & 118.7(9) \\
\hline$C(85)-C(84)-C(83)$ & $126.2(10)$ \\
\hline $\mathrm{C}(84)-\mathrm{C}(85)-\mathrm{C}(86)$ & 116.3(9) \\
\hline$C(81)-C(86)-C(85)$ & 120.1(9) \\
\hline $\mathrm{C}(92)-\mathrm{C}(87)-\mathrm{C}(88)$ & 119.1(8) \\
\hline $\mathrm{C}(92)-\mathrm{C}(87)-\mathrm{P}(4)$ & $121.5(6)$ \\
\hline $\mathrm{C}(88)-\mathrm{C}(87)-\mathrm{P}(4)$ & $119.4(7)$ \\
\hline $\mathrm{C}(87)-\mathrm{C}(88)-\mathrm{C}(89)$ & 119.8(10) \\
\hline C(90)-C(89)-C(88) & 118.0(10) \\
\hline C(89)-C(90)-C(91) & 121.8(9) \\
\hline $\mathrm{C}(90)-\mathrm{C}(91)-\mathrm{C}(92)$ & 123.4(9) \\
\hline $\mathrm{C}(91)-\mathrm{C}(92)-\mathrm{C}(87)$ & 117.8(8) \\
\hline $\mathrm{C}(94)-\mathrm{C}(93)-\mathrm{C}(102)$ & $121.5(10)$ \\
\hline $\mathrm{C}(94)-\mathrm{C}(93)-\mathrm{C}(103)$ & $120.2(9)$ \\
\hline $\mathrm{C}(102)-\mathrm{C}(93)-\mathrm{C}(103)$ & 117.7(8) \\
\hline $\mathrm{C}(93)-\mathrm{C}(94)-\mathrm{O}(9)$ & 122.1(9) \\
\hline C(93)-C(94)-C(95) & 121.4(9) \\
\hline $\mathrm{O}(9)-\mathrm{C}(94)-\mathrm{C}(95)$ & 116.5(9) \\
\hline$C(94)-C(95)-C(96)$ & 119.0(9) \\
\hline $\mathrm{C}(94)-\mathrm{C}(95)-\mathrm{C}(113)$ & 121.8(9) \\
\hline $\mathrm{C}(96)-\mathrm{C}(95)-\mathrm{C}(113)$ & 119.2(9) \\
\hline $\mathrm{C}(97)-\mathrm{C}(96)-\mathrm{C}(95)$ & 116.1(9) \\
\hline $\mathrm{C}(96)-\mathrm{C}(97)-\mathrm{C}(102)$ & $124.9(10)$ \\
\hline $\mathrm{C}(96)-\mathrm{C}(97)-\mathrm{C}(98)$ & $114.5(11)$ \\
\hline $\mathrm{C}(102)-\mathrm{C}(97)-\mathrm{C}(98)$ & $120.5(10)$ \\
\hline C(99)-C(98)-C(97) & $116.4(11)$ \\
\hline $\mathrm{C}(100)-\mathrm{C}(99)-\mathrm{C}(98)$ & $121.4(10)$ \\
\hline $\mathrm{C}(101)-\mathrm{C}(100)-\mathrm{C}(99)$ & $114.6(11)$ \\
\hline
\end{tabular}




\begin{tabular}{|c|c|}
\hline$C(100)-C(101)-C(102)$ & $130.5(11)$ \\
\hline $\mathrm{C}(97)-\mathrm{C}(102)-\mathrm{C}(101)$ & $116.4(9)$ \\
\hline C(97)-C(102)-C(93) & $116.9(10)$ \\
\hline $\mathrm{C}(101)-\mathrm{C}(102)-\mathrm{C}(93)$ & $126.7(10)$ \\
\hline$C(112)-C(103)-C(104)$ & 118.3(9) \\
\hline $\mathrm{C}(112)-\mathrm{C}(103)-\mathrm{C}(93)$ & $124.4(8)$ \\
\hline C(104)-C(103)-C(93) & 117.1(8) \\
\hline $\mathrm{O}(10)-\mathrm{C}(104)-\mathrm{C}(103)$ & 121.1(9) \\
\hline $\mathrm{O}(10)-\mathrm{C}(104)-\mathrm{C}(105)$ & $120.1(7)$ \\
\hline$C(103)-C(104)-C(105)$ & 118.8(8) \\
\hline$C(106)-C(105)-C(104)$ & $120.3(8)$ \\
\hline$C(106)-C(105)-C(126)$ & 124.0(9) \\
\hline$C(104)-C(105)-C(126)$ & $115.6(7)$ \\
\hline$C(105)-C(106)-C(107)$ & 118.8(9) \\
\hline$C(112)-C(107)-C(106)$ & 121.2(8) \\
\hline$C(112)-C(107)-C(108)$ & $121.7(10)$ \\
\hline$C(106)-C(107)-C(108)$ & 116.9(9) \\
\hline C(109)-C(108)-C(107) & 119.7(12) \\
\hline $\mathrm{C}(108)-\mathrm{C}(109)-\mathrm{C}(110)$ & $120.5(11)$ \\
\hline$C(111)-C(110)-C(109)$ & $115.2(11)$ \\
\hline$C(110)-C(111)-C(112)$ & $123.5(12)$ \\
\hline$C(107)-C(112)-C(103)$ & $122.4(8)$ \\
\hline $\mathrm{C}(107)-\mathrm{C}(112)-\mathrm{C}(111)$ & 119.3(9) \\
\hline $\mathrm{C}(103)-\mathrm{C}(112)-\mathrm{C}(111)$ & $118.3(10)$ \\
\hline $\mathrm{C}(95)-\mathrm{C}(113)-\mathrm{P}(5)$ & $116.8(7)$ \\
\hline$C(119)-C(114)-C(115)$ & $120.5(11)$ \\
\hline $\mathrm{C}(119)-\mathrm{C}(114)-\mathrm{P}(5)$ & 117.8(8) \\
\hline $\mathrm{C}(115)-\mathrm{C}(114)-\mathrm{P}(5)$ & $121.1(10)$ \\
\hline$C(116)-C(115)-C(114)$ & $122.1(12)$ \\
\hline$C(117)-C(116)-C(115)$ & $115.8(12)$ \\
\hline $\mathrm{C}(118)-\mathrm{C}(117)-\mathrm{C}(116)$ & $118.4(14)$ \\
\hline $\mathrm{C}(117)-\mathrm{C}(118)-\mathrm{C}(119)$ & $131.8(14)$ \\
\hline $\mathrm{C}(118)-\mathrm{C}(119)-\mathrm{C}(114)$ & 111.2(11) \\
\hline $\mathrm{C}(121)-\mathrm{C}(120)-\mathrm{C}(125)$ & 115.2(9) \\
\hline $\mathrm{C}(121)-\mathrm{C}(120)-\mathrm{P}(5)$ & $124.5(8)$ \\
\hline $\mathrm{C}(125)-\mathrm{C}(120)-\mathrm{P}(5)$ & 119.3(7) \\
\hline $\mathrm{C}(120)-\mathrm{C}(121)-\mathrm{C}(122)$ & $125.1(11)$ \\
\hline $\mathrm{C}(123)-\mathrm{C}(122)-\mathrm{C}(121)$ & $120.1(12)$ \\
\hline $\mathrm{C}(122)-\mathrm{C}(123)-\mathrm{C}(124)$ & $119.9(12)$ \\
\hline$C(125)-C(124)-C(123)$ & $116.5(12)$ \\
\hline$C(120)-C(125)-C(124)$ & $123.0(10)$ \\
\hline $\mathrm{C}(105)-\mathrm{C}(126)-\mathrm{P}(6)$ & $122.7(6)$ \\
\hline $\mathrm{C}(132)-\mathrm{C}(127)-\mathrm{C}(128)$ & 116.8(8) \\
\hline $\mathrm{C}(132)-\mathrm{C}(127)-\mathrm{P}(6)$ & $125.7(6)$ \\
\hline $\mathrm{C}(128)-\mathrm{C}(127)-\mathrm{P}(6)$ & $117.1(7)$ \\
\hline
\end{tabular}

\begin{tabular}{|c|c|}
\hline $\mathrm{C}(129)-\mathrm{C}(128)-\mathrm{C}(127)$ & $120.4(9)$ \\
\hline $\mathrm{C}(128)-\mathrm{C}(129)-\mathrm{C}(130)$ & 114.9(9) \\
\hline $\mathrm{C}(131)-\mathrm{C}(130)-\mathrm{C}(129)$ & 126.2(9) \\
\hline $\mathrm{C}(130)-\mathrm{C}(131)-\mathrm{C}(132)$ & 117.1(9) \\
\hline $\mathrm{C}(131)-\mathrm{C}(132)-\mathrm{C}(127)$ & $124.6(8)$ \\
\hline $\mathrm{C}(134)-\mathrm{C}(133)-\mathrm{C}(138)$ & 116.9(8) \\
\hline $\mathrm{C}(134)-\mathrm{C}(133)-\mathrm{P}(6)$ & 122.1(7) \\
\hline $\mathrm{C}(138)-\mathrm{C}(133)-\mathrm{P}(6)$ & $120.8(7)$ \\
\hline C(133)-C(134)-C(135) & 122.7(9) \\
\hline $\mathrm{C}(134)-\mathrm{C}(135)-\mathrm{C}(136)$ & 119.3(9) \\
\hline $\mathrm{C}(137)-\mathrm{C}(136)-\mathrm{C}(135)$ & $121.8(10)$ \\
\hline $\mathrm{C}(136)-\mathrm{C}(137)-\mathrm{C}(138)$ & $114.2(10)$ \\
\hline $\mathrm{C}(137)-\mathrm{C}(138)-\mathrm{C}(133)$ & $124.8(9)$ \\
\hline $\mathrm{O}(9)-\mathrm{La}(1)-\mathrm{O}(1)$ & $104.04(19)$ \\
\hline $\mathrm{O}(9)-\mathrm{La}(1)-\mathrm{O}(5)$ & 100.11(19) \\
\hline $\mathrm{O}(1)-\mathrm{La}(1)-\mathrm{O}(5)$ & $107.46(18)$ \\
\hline $\mathrm{O}(9)-\mathrm{La}(1)-\mathrm{O}(11)$ & 78.86(18) \\
\hline $\mathrm{O}(1)-\mathrm{La}(1)-\mathrm{O}(11)$ & $94.39(19)$ \\
\hline $\mathrm{O}(5)-\mathrm{La}(1)-\mathrm{O}(11)$ & $157.57(18)$ \\
\hline $\mathrm{O}(9)-\mathrm{La}(1)-\mathrm{O}(7)$ & $94.28(19)$ \\
\hline $\mathrm{O}(1)-\mathrm{La}(1)-\mathrm{O}(7)$ & 160.01(19) \\
\hline $\mathrm{O}(5)-\mathrm{La}(1)-\mathrm{O}(7)$ & $76.51(16)$ \\
\hline $\mathrm{O}(11)-\mathrm{La}(1)-\mathrm{O}(7)$ & 81.20(18) \\
\hline $\mathrm{O}(9)-\mathrm{La}(1)-\mathrm{O}(3)$ & $166.05(17)$ \\
\hline $\mathrm{O}(1)-\mathrm{La}(1)-\mathrm{O}(3)$ & $76.43(17)$ \\
\hline $\mathrm{O}(5)-\mathrm{La}(1)-\mathrm{O}(3)$ & 92.91(18) \\
\hline $\mathrm{O}(11)-\mathrm{La}(1)-\mathrm{O}(3)$ & $87.20(17)$ \\
\hline $\mathrm{O}(7)-\mathrm{La}(1)-\mathrm{O}(3)$ & 83.86(18) \\
\hline $\mathrm{O}(10)-\mathrm{La}(2)-\mathrm{O}(6)$ & $104.23(18)$ \\
\hline $\mathrm{O}(10)-\mathrm{La}(2)-\mathrm{O}(2)$ & 107.68(18) \\
\hline $\mathrm{O}(6)-\mathrm{La}(2)-\mathrm{O}(2)$ & $102.03(18)$ \\
\hline $\mathrm{O}(10)-\mathrm{La}(2)-\mathrm{O}(4)$ & $91.71(17)$ \\
\hline $\mathrm{O}(6)-\mathrm{La}(2)-\mathrm{O}(4)$ & $163.18(17)$ \\
\hline $\mathrm{O}(2)-\mathrm{La}(2)-\mathrm{O}(4)$ & 77.66(18) \\
\hline $\mathrm{O}(10)-\mathrm{La}(2)-\mathrm{O}(8)$ & $158.65(18)$ \\
\hline $\mathrm{O}(6)-\mathrm{La}(2)-\mathrm{O}(8)$ & 79.01(17) \\
\hline $\mathrm{O}(2)-\mathrm{La}(2)-\mathrm{O}(8)$ & 91.93(18) \\
\hline $\mathrm{O}(4)-\mathrm{La}(2)-\mathrm{O}(8)$ & $84.18(16)$ \\
\hline $\mathrm{O}(10)-\mathrm{La}(2)-\mathrm{O}(12)$ & $76.34(16)$ \\
\hline $\mathrm{O}(6)-\mathrm{La}(2)-\mathrm{O}(12)$ & $90.69(18)$ \\
\hline $\mathrm{O}(2)-\mathrm{La}(2)-\mathrm{O}(12)$ & $164.97(18)$ \\
\hline $\mathrm{O}(4)-\mathrm{La}(2)-\mathrm{O}(12)$ & 87.84(18) \\
\hline $\mathrm{O}(8)-\mathrm{La}(2)-\mathrm{O}(12)$ & $82.57(17)$ \\
\hline $\mathrm{C}(2)-\mathrm{O}(1)-\mathrm{La}(1)$ & $167.8(5)$ \\
\hline $\mathrm{C}(12)-\mathrm{O}(2)-\mathrm{La}(2)$ & $163.4(6)$ \\
\hline
\end{tabular}




$\begin{array}{llll}\mathrm{P}(1)-\mathrm{O}(3)-\mathrm{La}(1) & 129.2(3) & \mathrm{O}(7)-\mathrm{P}(3)-\mathrm{C}(74) & 106.2(4) \\ \mathrm{P}(2)-\mathrm{O}(4)-\mathrm{La}(2) & 130.8(3) & \mathrm{C}(67)-\mathrm{P}(3)-\mathrm{C}(74) & 110.0(4) \\ \mathrm{C}(48)-\mathrm{O}(5)-\mathrm{La}(1) & 167.2(5) & \mathrm{O}(7)-\mathrm{P}(3)-\mathrm{C}(68) & 112.0(4) \\ \mathrm{C}(58)-\mathrm{O}(6)-\mathrm{La}(2) & 165.5(5) & \mathrm{C}(67)-\mathrm{P}(3)-\mathrm{C}(68) & 105.1(4) \\ \mathrm{P}(3)-\mathrm{O}(7)-\mathrm{La}(1) & 125.6(3) & \mathrm{C}(74)-\mathrm{P}(3)-\mathrm{C}(68) & 107.0(4) \\ \mathrm{P}(4)-\mathrm{O}(8)-\mathrm{La}(2) & 127.3(3) & \mathrm{O}(8)-\mathrm{P}(4)-\mathrm{C}(81) & 110.8(4) \\ \mathrm{C}(94)-\mathrm{O}(9)-\mathrm{La}(1) & 164.4(6) & \mathrm{O}(8)-\mathrm{P}(4)-\mathrm{C}(87) & 109.1(3) \\ \mathrm{C}(104)-\mathrm{O}(10)-\mathrm{La}(2) & 162.0(5) & \mathrm{C}(81)-\mathrm{P}(4)-\mathrm{C}(87) & 108.5(4) \\ \mathrm{P}(5)-\mathrm{O}(11)-\mathrm{La}(1) & 128.7(3) & \mathrm{O}(8)-\mathrm{P}(4)-\mathrm{C}(80) & 115.5(3) \\ \mathrm{P}(6)-\mathrm{O}(12)-\mathrm{La}(2) & 128.0(3) & \mathrm{C}(81)-\mathrm{P}(4)-\mathrm{C}(80) & 106.9(4) \\ \mathrm{O}(3)-\mathrm{P}(1)-\mathrm{C}(28) & 107.9(4) & \mathrm{C}(87)-\mathrm{P}(4)-\mathrm{C}(80) & 105.6(4) \\ \mathrm{O}(3)-\mathrm{P}(1)-\mathrm{C}(22) & 109.5(4) & \mathrm{O}(11)-\mathrm{P}(5)-\mathrm{C}(113) & 113.6(4) \\ \mathrm{C}(28)-\mathrm{P}(1)-\mathrm{C}(22) & 105.2(4) & \mathrm{O}(11)-\mathrm{P}(5)-\mathrm{C}(120) & 110.1(4) \\ \mathrm{O}(3)-\mathrm{P}(1)-\mathrm{C}(21) & 115.8(4) & \mathrm{C}(113)-\mathrm{P}(5)-\mathrm{C}(120) & 108.0(5) \\ \mathrm{C}(28)-\mathrm{P}(1)-\mathrm{C}(21) & 107.8(5) & \mathrm{O}(11)-\mathrm{P}(5)-\mathrm{C}(114) & 110.8(4) \\ \mathrm{C}(22)-\mathrm{P}(1)-\mathrm{C}(21) & 110.1(5) & \mathrm{C}(113)-\mathrm{P}(5)-\mathrm{C}(114) & 107.4(4) \\ \mathrm{O}(4)-\mathrm{P}(2)-\mathrm{C}(35) & 109.2(3) & \mathrm{C}(120)-\mathrm{P}(5)-\mathrm{C}(114) & 106.6(4) \\ \mathrm{O}(4)-\mathrm{P}(2)-\mathrm{C}(41) & 111.3(4) & \mathrm{O}(12)-\mathrm{P}(6)-\mathrm{C}(133) & 108.9(3) \\ \mathrm{C}(35)-\mathrm{P}(2)-\mathrm{C}(41) & 107.9(4) & \mathrm{O}(12)-\mathrm{P}(6)-\mathrm{C}(127) & 114.0(4) \\ \mathrm{O}(4)-\mathrm{P}(2)-\mathrm{C}(34) & 114.3(4) & \mathrm{C}(133)-\mathrm{P}(6)-\mathrm{C}(127) & 105.7(4) \\ \mathrm{C}(35)-\mathrm{P}(2)-\mathrm{C}(34) & 107.0(4) & \mathrm{O}(12)-\mathrm{P}(6)-\mathrm{C}(126) & 113.8(4) \\ \mathrm{C}(41)-\mathrm{P}(2)-\mathrm{C}(34) & 106.9(4) & \mathrm{C}(133)-\mathrm{P}(6)-\mathrm{C}(126) & 107.2(4) \\ \mathrm{O}(7)-\mathrm{P}(3)-\mathrm{C}(67) & 116.2(4) & \mathrm{C}(127)-\mathrm{P}(6)-\mathrm{C}(126) & 106.8(4) \\ & & & \end{array}$

Symmetry transformations used to generate equivalent atoms: 
Table S19. Anisotropic displacement parameters $\left(\AA^{2} \times 10^{3}\right)$ for $\mathbf{8}$. The anisotropic displacement factor exponent takes the form: $-2 \pi^{2}\left[\mathrm{~h}^{2} \mathrm{a}^{* 2} \mathrm{U}^{11}+\ldots+2 \mathrm{hka} \mathrm{b}^{*} \mathrm{U}^{12}\right]$

\begin{tabular}{|c|c|c|c|c|c|c|}
\hline & $\mathrm{U}^{11}$ & $\mathrm{U}^{22}$ & $\mathrm{U}^{33}$ & $\mathrm{U}^{23}$ & $\mathrm{U}^{13}$ & $\mathrm{U}^{12}$ \\
\hline$\overline{C(1)}$ & $37(6)$ & $42(6)$ & $20(4)$ & $-3(4)$ & $17(4)$ & $18(5)$ \\
\hline $\mathrm{C}(2)$ & $37(6)$ & $51(6)$ & $24(4)$ & $-6(4)$ & $-2(4)$ & 22(5) \\
\hline C(3) & $16(5)$ & $34(6)$ & $62(6)$ & $6(5)$ & $14(4)$ & $11(4)$ \\
\hline C(4) & $44(6)$ & $43(6)$ & $47(6)$ & $-4(4)$ & $-19(5)$ & 23(5) \\
\hline C(5) & $49(6)$ & $81(7)$ & $27(4)$ & $-21(6)$ & $-8(5)$ & $40(6)$ \\
\hline$C(6)$ & 69(8) & $48(7)$ & $57(6)$ & $-16(5)$ & $-2(6)$ & $25(6)$ \\
\hline$C(7)$ & $66(8)$ & $95(9)$ & $47(6)$ & $0(6)$ & $-3(6)$ & $57(8)$ \\
\hline$C(8)$ & $46(7)$ & $92(9)$ & $77(8)$ & $-47(7)$ & $-15(6)$ & $38(7)$ \\
\hline C(9) & $53(6)$ & $47(6)$ & $34(5)$ & $2(5)$ & $21(5)$ & $35(5)$ \\
\hline$C(10)$ & $15(5)$ & $40(6)$ & $41(5)$ & $0(4)$ & $12(4)$ & $7(4)$ \\
\hline $\mathrm{C}(11)$ & $39(6)$ & $39(6)$ & $27(4)$ & $0(4)$ & $-2(4)$ & 20(5) \\
\hline$C(12)$ & $32(6)$ & $41(6)$ & $23(5)$ & $-1(4)$ & $9(4)$ & $-4(5)$ \\
\hline$C(13)$ & $56(6)$ & $39(6)$ & $20(4)$ & 6(4) & $8(4)$ & 11(5) \\
\hline$C(14)$ & $76(8)$ & $86(9)$ & $22(5)$ & $-6(5)$ & $8(5)$ & $9(7)$ \\
\hline $\mathrm{C}(15)$ & $62(7)$ & $55(7)$ & $41(6)$ & $-8(5)$ & $24(5)$ & $25(6)$ \\
\hline$C(16)$ & $77(8)$ & $128(11)$ & $38(6)$ & $-20(6)$ & $12(6)$ & 61(9) \\
\hline $\mathrm{C}(17)$ & $41(7)$ & $108(10)$ & $25(5)$ & $-1(6)$ & $7(5)$ & $12(7)$ \\
\hline C(18) & $64(8)$ & $68(8)$ & $70(8)$ & 1(6) & $20(6)$ & $24(6)$ \\
\hline C(19) & $49(6)$ & 83(8) & $37(6)$ & $-4(5)$ & $10(5)$ & $28(6)$ \\
\hline C(20) & $22(5)$ & $70(7)$ & $35(5)$ & $-11(5)$ & 1(4) & 19(5) \\
\hline$C(21)$ & $65(7)$ & $60(7)$ & $43(5)$ & $-16(5)$ & 2(5) & $41(6)$ \\
\hline$C(22)$ & $51(7)$ & $79(8)$ & $87(9)$ & $7(6)$ & $35(6)$ & $52(6)$ \\
\hline$C(23)$ & $119(12)$ & $142(13)$ & $166(14)$ & $78(11)$ & 131(11) & $86(11)$ \\
\hline$C(24)$ & $290(30)$ & $290(30)$ & $390(40)$ & 150(30) & $180(30)$ & $270(30)$ \\
\hline$C(25)$ & $180(20)$ & $78(13)$ & $240(20)$ & $46(14)$ & $90(20)$ & 10(13) \\
\hline$C(26)$ & $51(9)$ & 124(13) & $250(20)$ & $-141(14)$ & $-17(11)$ & 33(9) \\
\hline C(27) & $50(7)$ & $174(12)$ & $84(8)$ & $-73(9)$ & $-5(7)$ & $70(8)$ \\
\hline C(28) & $34(6)$ & $49(6)$ & 83(8) & $3(6)$ & 1(6) & $14(5)$ \\
\hline C(29) & 204(17) & $59(9)$ & $72(9)$ & $-39(7)$ & $-6(10)$ & $17(10)$ \\
\hline C(30) & 189(17) & $60(11)$ & $114(12)$ & $-32(9)$ & $52(12)$ & $6(11)$ \\
\hline C(31) & $150(14)$ & $41(8)$ & 158(15) & $-39(12)$ & $-37(15)$ & 1(9) \\
\hline C(32) & $169(15)$ & $31(8)$ & $108(11)$ & $3(8)$ & $-35(11)$ & $-36(9)$ \\
\hline C(33) & $119(12)$ & $72(10)$ & $113(12)$ & 5(9) & $-9(10)$ & 16(9) \\
\hline C(34) & $52(6)$ & $37(5)$ & $27(5)$ & $15(4)$ & $6(4)$ & $7(5)$ \\
\hline C(35) & $32(5)$ & $33(5)$ & $22(5)$ & $-1(4)$ & $0(4)$ & $7(4)$ \\
\hline$C(36)$ & $124(10)$ & $79(9)$ & 4(4) & $-3(5)$ & $-10(5)$ & $20(8)$ \\
\hline C(37) & $80(8)$ & $47(7)$ & $52(6)$ & $-23(5)$ & $-23(6)$ & 28(6) \\
\hline C(38) & $85(8)$ & $41(6)$ & $52(6)$ & $10(6)$ & $3(7)$ & $14(6)$ \\
\hline C(39) & $83(8)$ & $54(7)$ & $34(5)$ & $-2(5)$ & 1(5) & $37(7)$ \\
\hline$C(40)$ & $70(7)$ & $51(7)$ & $19(4)$ & 1(4) & 5(4) & $25(6)$ \\
\hline
\end{tabular}




\begin{tabular}{|c|c|c|c|c|c|c|}
\hline $\mathrm{C}(41)$ & $55(7)$ & $49(6)$ & $44(6)$ & $-8(5)$ & $10(5)$ & $29(6)$ \\
\hline$C(42)$ & $62(7)$ & $57(7)$ & $68(7)$ & $9(5)$ & 12(6) & $40(6)$ \\
\hline$C(43)$ & $58(8)$ & $66(9)$ & $135(11)$ & $7(8)$ & $-11(8)$ & $36(7)$ \\
\hline$C(44)$ & 61(9) & 68(9) & $130(11)$ & $-46(8)$ & $-27(8)$ & 26(8) \\
\hline$C(45)$ & $78(9)$ & $98(10)$ & $49(6)$ & 1(6) & $-17(6)$ & 51(8) \\
\hline$C(46)$ & $53(7)$ & $67(7)$ & $44(6)$ & $-2(5)$ & $10(5)$ & $35(6)$ \\
\hline$C(47)$ & $23(5)$ & $24(5)$ & $21(4)$ & $-3(3)$ & 1(3) & $5(4)$ \\
\hline$C(48)$ & $19(5)$ & $35(5)$ & $38(5)$ & $-6(4)$ & $3(4)$ & $12(4)$ \\
\hline$C(49)$ & $43(6)$ & $30(5)$ & $33(5)$ & $-9(4)$ & $-6(4)$ & $14(5)$ \\
\hline$C(50)$ & $54(6)$ & $40(6)$ & $32(5)$ & $-9(4)$ & $-3(5)$ & $24(5)$ \\
\hline$C(51)$ & $34(5)$ & $25(5)$ & $32(5)$ & $-13(4)$ & 1(4) & $-2(4)$ \\
\hline$C(52)$ & $40(5)$ & $49(6)$ & $45(6)$ & $-13(5)$ & $-20(5)$ & $22(5)$ \\
\hline$C(53)$ & $43(6)$ & $48(6)$ & $57(6)$ & $-6(5)$ & $-3(5)$ & $34(5)$ \\
\hline$C(54)$ & $58(7)$ & $29(6)$ & $63(6)$ & $14(5)$ & $33(6)$ & $5(5)$ \\
\hline C(55) & $46(6)$ & $48(6)$ & $31(5)$ & $19(4)$ & $25(4)$ & $29(5)$ \\
\hline$C(56)$ & $23(4)$ & $24(4)$ & $39(5)$ & $3(5)$ & $5(5)$ & $8(4)$ \\
\hline$C(57)$ & $23(4)$ & $44(5)$ & $20(4)$ & $-2(5)$ & $-8(4)$ & $2(4)$ \\
\hline$C(58)$ & $64(6)$ & $47(6)$ & $21(4)$ & $11(4)$ & $16(4)$ & $46(5)$ \\
\hline$C(59)$ & $29(5)$ & $48(6)$ & $34(5)$ & $23(4)$ & $6(4)$ & $22(5)$ \\
\hline$C(60)$ & $70(7)$ & $41(6)$ & $32(5)$ & $19(4)$ & $6(5)$ & $29(6)$ \\
\hline $\mathrm{C}(61)$ & $49(6)$ & $40(6)$ & $39(5)$ & $9(5)$ & $10(5)$ & $6(5)$ \\
\hline$C(62)$ & $78(8)$ & $19(5)$ & $58(6)$ & $-2(5)$ & $-5(6)$ & $14(6)$ \\
\hline C(63) & $59(8)$ & $46(7)$ & $68(7)$ & $-8(5)$ & $-8(6)$ & 18(6) \\
\hline C(64) & $47(6)$ & $54(6)$ & $61(6)$ & $10(6)$ & $2(6)$ & $28(5)$ \\
\hline C(65) & $39(5)$ & $30(5)$ & $30(5)$ & $9(4)$ & $11(4)$ & $24(4)$ \\
\hline$C(66)$ & $26(5)$ & $28(5)$ & $42(5)$ & $-15(4)$ & $-5(4)$ & $2(4)$ \\
\hline$C(67)$ & $59(6)$ & $28(5)$ & $31(5)$ & $-10(5)$ & $-18(5)$ & $2(4)$ \\
\hline$C(68)$ & $47(6)$ & $54(6)$ & $22(4)$ & $-19(4)$ & $-16(4)$ & $21(5)$ \\
\hline $\mathrm{C}(69)$ & $43(6)$ & $44(6)$ & $36(5)$ & $-1(4)$ & $-1(4)$ & $26(5)$ \\
\hline$C(70)$ & $72(7)$ & $75(8)$ & $47(6)$ & $-2(6)$ & $-6(5)$ & $57(7)$ \\
\hline $\mathrm{C}(71)$ & $49(6)$ & $40(6)$ & $16(5)$ & $4(4)$ & $-6(4)$ & $-3(5)$ \\
\hline $\mathrm{C}(72)$ & $60(7)$ & $46(6)$ & $60(6)$ & $-11(6)$ & $-32(6)$ & $34(5)$ \\
\hline $\mathrm{C}(73)$ & $63(7)$ & $37(6)$ & $36(5)$ & $1(4)$ & $-5(5)$ & $22(6)$ \\
\hline$C(74)$ & $61(6)$ & $47(6)$ & $22(4)$ & $0(4)$ & $3(4)$ & $36(5)$ \\
\hline$C(75)$ & 103(10) & $75(8)$ & $59(7)$ & $-27(6)$ & $-14(7)$ & $50(8)$ \\
\hline$C(76)$ & $76(8)$ & $79(9)$ & $27(5)$ & $-20(6)$ & $-17(5)$ & $13(7)$ \\
\hline$C(77)$ & $87(8)$ & $47(7)$ & $45(6)$ & $17(5)$ & $-14(6)$ & $12(6)$ \\
\hline$C(78)$ & 149(11) & $66(8)$ & $34(6)$ & $1(6)$ & $-43(6)$ & $59(8)$ \\
\hline $\mathrm{C}(79)$ & 111(10) & $93(9)$ & $31(6)$ & $-23(6)$ & $-19(6)$ & $46(8)$ \\
\hline $\mathrm{C}(80)$ & $27(5)$ & $30(5)$ & $44(5)$ & $-3(4)$ & $-3(4)$ & $13(4)$ \\
\hline $\mathrm{C}(81)$ & $10(5)$ & $50(6)$ & $24(4)$ & $6(4)$ & 11(3) & $-1(4)$ \\
\hline $\mathrm{C}(82)$ & $32(6)$ & $61(7)$ & $27(5)$ & $15(4)$ & $9(4)$ & $25(5)$ \\
\hline$C(83)$ & $36(6)$ & $75(8)$ & $49(6)$ & $-20(6)$ & $-13(5)$ & $31(6)$ \\
\hline$C(84)$ & $49(7)$ & $54(7)$ & $62(7)$ & $4(6)$ & $13(6)$ & $25(6)$ \\
\hline$C(85)$ & $76(8)$ & $47(7)$ & $60(7)$ & $32(5)$ & $27(6)$ & $23(6)$ \\
\hline
\end{tabular}




\begin{tabular}{|c|c|c|c|c|c|c|}
\hline$C(86)$ & $41(6)$ & $51(6)$ & $58(6)$ & $16(5)$ & $26(5)$ & $8(5)$ \\
\hline $\mathrm{C}(87)$ & $45(6)$ & $38(5)$ & $15(4)$ & $9(4)$ & $4(4)$ & $17(5)$ \\
\hline C(88) & $52(6)$ & $52(6)$ & $35(5)$ & $-5(5)$ & $-17(5)$ & $19(5)$ \\
\hline C(89) & $124(11)$ & 133(11) & $48(7)$ & $-20(7)$ & $-71(7)$ & $79(10)$ \\
\hline C(90) & $43(7)$ & 121(10) & $46(6)$ & $-44(6)$ & $-23(5)$ & $50(7)$ \\
\hline $\mathrm{C}(91)$ & $33(6)$ & $124(10)$ & $49(6)$ & $-27(6)$ & $-6(5)$ & $55(6)$ \\
\hline $\mathrm{C}(92)$ & $41(6)$ & $68(7)$ & $67(7)$ & $-13(6)$ & $-10(5)$ & $32(6)$ \\
\hline C(93) & 110(9) & $77(7)$ & $16(4)$ & $-8(5)$ & $-16(6)$ & $69(7)$ \\
\hline C(94) & $59(7)$ & $42(6)$ & $20(5)$ & $-3(4)$ & $-4(4)$ & $18(6)$ \\
\hline C(95) & $74(7)$ & $36(6)$ & $29(5)$ & $-1(4)$ & $5(5)$ & $22(6)$ \\
\hline $\mathrm{C}(96)$ & $91(8)$ & $90(9)$ & $8(4)$ & $6(5)$ & $6(5)$ & $40(8)$ \\
\hline $\mathrm{C}(97)$ & $140(11)$ & $38(6)$ & $15(5)$ & $-1(4)$ & $17(6)$ & $42(7)$ \\
\hline C(98) & 199(14) & $62(8)$ & $18(5)$ & $16(5)$ & $11(7)$ & 71(10) \\
\hline C(99) & $112(10)$ & $33(6)$ & $64(7)$ & $-20(5)$ & $-40(7)$ & $33(7)$ \\
\hline$C(100)$ & $70(7)$ & 85(9) & $30(5)$ & $7(6)$ & $8(6)$ & $33(7)$ \\
\hline C(101) & $98(8)$ & $44(6)$ & $37(5)$ & $0(6)$ & $-18(7)$ & $28(6)$ \\
\hline $\mathrm{C}(102)$ & $91(8)$ & $26(5)$ & $14(5)$ & $13(4)$ & $6(5)$ & $30(6)$ \\
\hline C(103) & $73(7)$ & $30(5)$ & $43(6)$ & $-8(4)$ & $-5(5)$ & $27(5)$ \\
\hline C(104) & $68(7)$ & $36(5)$ & $24(5)$ & $-11(4)$ & $-27(5)$ & $30(5)$ \\
\hline$C(105)$ & $42(6)$ & $61(7)$ & 21(4) & $-18(4)$ & $-9(4)$ & $29(5)$ \\
\hline$C(106)$ & $65(7)$ & $67(7)$ & $42(6)$ & $-33(5)$ & $-22(5)$ & $35(6)$ \\
\hline C(107) & $55(6)$ & $85(7)$ & $49(6)$ & $-37(6)$ & $-30(6)$ & $58(6)$ \\
\hline C(108) & $96(9)$ & $81(8)$ & $66(7)$ & $-34(6)$ & $-11(7)$ & $68(8)$ \\
\hline C(109) & 241(19) & 238(18) & $26(6)$ & $-42(8)$ & $-41(9)$ & 218(17) \\
\hline$C(110)$ & $228(18)$ & $71(9)$ & $48(7)$ & $-33(7)$ & $-36(10)$ & 93(11) \\
\hline $\mathrm{C}(111)$ & 107(9) & $62(7)$ & $61(7)$ & $-24(5)$ & $-64(7)$ & $57(7)$ \\
\hline $\mathrm{C}(112)$ & 104(9) & $56(6)$ & $17(4)$ & $-16(4)$ & $-19(5)$ & $71(7)$ \\
\hline $\mathrm{C}(113)$ & $59(7)$ & $44(6)$ & $36(5)$ & $-1(5)$ & $1(5)$ & $8(5)$ \\
\hline$C(114)$ & $118(10)$ & $27(6)$ & $16(5)$ & $-10(4)$ & $26(5)$ & $19(7)$ \\
\hline $\mathrm{C}(115)$ & 104(10) & $52(8)$ & $67(7)$ & $-19(6)$ & $6(7)$ & $48(8)$ \\
\hline$C(116)$ & $100(11)$ & $97(10)$ & $44(6)$ & 19(6) & $-7(6)$ & $68(9)$ \\
\hline $\mathrm{C}(117)$ & $64(9)$ & $118(12)$ & $55(7)$ & $17(8)$ & $-9(7)$ & $22(9)$ \\
\hline C(118) & $120(12)$ & $125(12)$ & $46(7)$ & $17(7)$ & $-34(7)$ & $69(10)$ \\
\hline C(119) & $77(8)$ & $60(8)$ & $13(5)$ & $-5(5)$ & $1(5)$ & $20(7)$ \\
\hline $\mathrm{C}(120)$ & $55(7)$ & $53(6)$ & $28(5)$ & $2(4)$ & $-3(5)$ & $26(5)$ \\
\hline $\mathrm{C}(121)$ & $116(10)$ & $99(9)$ & $30(5)$ & $10(5)$ & $17(6)$ & $87(8)$ \\
\hline$C(122)$ & $114(12)$ & $220(17)$ & $44(7)$ & $16(8)$ & $4(7)$ & 121(12) \\
\hline $\mathrm{C}(123)$ & $104(11)$ & $56(8)$ & $79(9)$ & $1(7)$ & $35(8)$ & $36(8)$ \\
\hline$C(124)$ & $53(8)$ & $116(11)$ & $88(10)$ & $-53(8)$ & $-4(7)$ & 21(8) \\
\hline$C(125)$ & $78(9)$ & $61(7)$ & 59(7) & $-14(6)$ & $10(7)$ & $26(7)$ \\
\hline$C(126)$ & $48(6)$ & $38(5)$ & $48(6)$ & $-17(4)$ & $-9(4)$ & $21(5)$ \\
\hline $\mathrm{C}(127)$ & $23(5)$ & $20(5)$ & $42(5)$ & $-15(4)$ & $-7(4)$ & $4(4)$ \\
\hline C(128) & $64(7)$ & $61(7)$ & $23(4)$ & $-8(4)$ & $-7(4)$ & $36(6)$ \\
\hline C(129) & $69(7)$ & $31(6)$ & 101(8) & $-3(7)$ & $-13(7)$ & $19(5)$ \\
\hline $\mathrm{C}(130)$ & $110(10)$ & $44(6)$ & $53(7)$ & $23(5)$ & $34(6)$ & $63(7)$ \\
\hline
\end{tabular}




\begin{tabular}{lcccccc}
$\mathrm{C}(131)$ & $10(5)$ & $68(7)$ & $56(6)$ & $-5(5)$ & $-10(4)$ & $13(5)$ \\
$\mathrm{C}(132)$ & $43(5)$ & $43(6)$ & $45(5)$ & $-9(5)$ & $-22(5)$ & $23(5)$ \\
$\mathrm{C}(133)$ & $40(6)$ & $26(5)$ & $26(5)$ & $-1(4)$ & $-1(4)$ & $13(5)$ \\
$\mathrm{C}(134)$ & $37(6)$ & $34(6)$ & $69(7)$ & $11(5)$ & $14(5)$ & $14(5)$ \\
$\mathrm{C}(135)$ & $77(8)$ & $97(9)$ & $23(5)$ & $8(5)$ & $14(5)$ & $40(7)$ \\
$\mathrm{C}(136)$ & $47(7)$ & $55(7)$ & $75(8)$ & $48(6)$ & $22(6)$ & $18(6)$ \\
$\mathrm{C}(137)$ & $126(11)$ & $87(9)$ & $54(7)$ & $-13(7)$ & $22(7)$ & $65(9)$ \\
$\mathrm{C}(138)$ & $85(8)$ & $67(8)$ & $54(6)$ & $-21(6)$ & $-32(6)$ & $59(7)$ \\
$\mathrm{La}(1)$ & $44(1)$ & $41(1)$ & $23(1)$ & $1(1)$ & $1(1)$ & $20(1)$ \\
$\mathrm{La}(2)$ & $40(1)$ & $36(1)$ & $23(1)$ & $0(1)$ & $2(1)$ & $18(1)$ \\
$\mathrm{O}(1)$ & $43(4)$ & $29(3)$ & $47(4)$ & $1(3)$ & $5(3)$ & $10(3)$ \\
$\mathrm{O}(2)$ & $53(4)$ & $30(3)$ & $29(3)$ & $8(3)$ & $11(3)$ & $10(3)$ \\
$\mathrm{O}(3)$ & $44(4)$ & $55(4)$ & $39(4)$ & $-6(3)$ & $-8(3)$ & $30(3)$ \\
$\mathrm{O}(4)$ & $53(4)$ & $59(4)$ & $27(3)$ & $19(3)$ & $9(3)$ & $30(3)$ \\
$\mathrm{O}(5)$ & $50(4)$ & $43(4)$ & $32(3)$ & $1(3)$ & $-8(3)$ & $22(3)$ \\
$\mathrm{O}(6)$ & $37(3)$ & $50(4)$ & $30(3)$ & $-13(3)$ & $2(3)$ & $20(3)$ \\
$\mathrm{O}(7)$ & $45(4)$ & $42(4)$ & $41(4)$ & $1(3)$ & $1(3)$ & $9(3)$ \\
$\mathrm{O}(8)$ & $36(3)$ & $30(3)$ & $39(3)$ & $-1(3)$ & $-10(3)$ & $8(3)$ \\
$\mathrm{O}(9)$ & $67(4)$ & $65(4)$ & $22(3)$ & $1(3)$ & $-3(3)$ & $39(4)$ \\
$\mathrm{O}(10)$ & $62(4)$ & $36(4)$ & $23(3)$ & $-1(3)$ & $4(3)$ & $18(3)$ \\
$\mathrm{O}(11)$ & $60(4)$ & $40(4)$ & $19(3)$ & $-4(3)$ & $6(3)$ & $16(3)$ \\
$\mathrm{O}(12)$ & $32(3)$ & $60(4)$ & $27(3)$ & $-6(3)$ & $-7(3)$ & $28(3)$ \\
$\mathrm{P}(1)$ & $43(2)$ & $43(2)$ & $51(2)$ & $-11(1)$ & $-2(1)$ & $24(1)$ \\
$\mathrm{P}(2)$ & $49(2)$ & $42(1)$ & $22(1)$ & $-1(1)$ & $3(1)$ & $20(1)$ \\
$\mathrm{P}(3)$ & $53(2)$ & $43(2)$ & $22(1)$ & $-8(1)$ & $-10(1)$ & $23(1)$ \\
$\mathrm{P}(4)$ & $36(1)$ & $41(2)$ & $24(1)$ & $1(1)$ & $-2(1)$ & $18(1)$ \\
$\mathrm{P}(5)$ & $68(2)$ & $43(2)$ & $22(1)$ & $1(1)$ & $6(1)$ & $25(2)$ \\
$\mathrm{P}(6)$ & $45(2)$ & $42(2)$ & $30(1)$ & $-8(1)$ & $-5(1)$ & $25(1)$ \\
& & & & & & \\
\hline & & & & & & \\
\end{tabular}


Table S20. Hydrogen coordinates $\left(\times 10^{4}\right)$ and isotropic displacement parameters $\left(\AA^{2} \times 10^{3}\right)$ for 8.

\begin{tabular}{lrrrr}
\hline & $\mathrm{x}$ & $\mathrm{y}$ & $\mathrm{z}$ & $\mathrm{U}(\mathrm{eq})$ \\
\hline $\mathrm{H}(4)$ & -463 & 7524 & 4289 & 53 \\
$\mathrm{H}(6)$ & -1202 & 7176 & 3720 & 72 \\
$\mathrm{H}(7)$ & -1923 & 6552 & 3397 & 74 \\
$\mathrm{H}(8)$ & -2165 & 5779 & 3795 & 85 \\
$\mathrm{H}(9)$ & -1717 & 5653 & 4390 & 48 \\
$\mathrm{H}(14)$ & -1671 & 4512 & 5602 & 91 \\
$\mathrm{H}(16)$ & -2213 & 4631 & 6143 & 92 \\
$\mathrm{H}(17)$ & -2466 & 5150 & 6445 & 83 \\
$\mathrm{H}(18)$ & -2183 & 5916 & 6077 & 86 \\
$\mathrm{H}(19)$ & -1529 & 6233 & 5506 & 70 \\
$\mathrm{H}(21 \mathrm{~A})$ & 228 & 7840 & 4750 & 62 \\
$\mathrm{H}(21 \mathrm{~B})$ & 427 & 7482 & 4800 & 62 \\
$\mathrm{H}(23)$ & -128 & 7238 & 6524 & 160 \\
$\mathrm{H}(24)$ & -792 & 6966 & 7031 & 319 \\
$\mathrm{H}(25)$ & -1314 & 7139 & 6645 & 229 \\
$\mathrm{H}(26)$ & -1216 & 7628 & 5960 & 178 \\
$\mathrm{H}(27)$ & -459 & 7904 & 5489 & 115 \\
$\mathrm{H}(29)$ & 492 & 8404 & 6423 & 160 \\
$\mathrm{H}(30)$ & 1049 & 9228 & 6490 & 175 \\
$\mathrm{H}(31)$ & 1513 & 9660 & 5796 & 164 \\
$\mathrm{H}(32)$ & 1495 & 9295 & 5024 & 169 \\
$\mathrm{H}(33)$ & 1019 & 8438 & 4987 & 139 \\
$\mathrm{H}(34 \mathrm{~A})$ & -1098 & 4375 & 5172 & 55 \\
$\mathrm{H}(34 \mathrm{~B})$ & -575 & 4822 & 5118 & 55 \\
$\mathrm{H}(36)$ & -741 & 3892 & 5050 & 99 \\
$\mathrm{H}(37)$ & -815 & 3110 & 5014 & 74 \\
$\mathrm{H}(38)$ & -1008 & 2721 & 4228 & 81 \\
$\mathrm{H}(39)$ & -1201 & 3056 & 3492 & 67 \\
$\mathrm{H}(40)$ & -1196 & 3763 & 3549 & 59 \\
$\mathrm{H}(42)$ & -1908 & 3809 & 4265 & 69 \\
$\mathrm{H}(43)$ & -2564 & 3749 & 3830 & 101 \\
$\mathrm{H}(44)$ & -2472 & 4363 & 3252 & 107 \\
$\mathrm{H}(45)$ & -1693 & 5040 & 3129 & 87 \\
$\mathrm{H}(46)$ & -1039 & 5139 & 3617 & 63 \\
$\mathrm{H}(50)$ & 2500 & 6980 & 5123 & 50 \\
$\mathrm{H}(52)$ & 2985 & 7198 & 4306 & 54 \\
$\mathrm{H}(53)$ & 3109 & 7461 & 3466 & 53 \\
$\mathrm{H}(54)$ & 2558 & 7618 & 3035 & 69 \\
$\mathrm{H}(55)$ & 1893 & 7536 & 3457 & 47 \\
$\mathrm{H}(60)$ & 387 & 7691 & 3520 & 57 \\
& & & &
\end{tabular}




\begin{tabular}{|c|c|c|c|c|}
\hline $\mathrm{H}(62)$ & 1029 & 8580 & 3597 & 67 \\
\hline H(63) & 1801 & 9139 & 3819 & 73 \\
\hline $\mathrm{H}(64)$ & 2249 & 8967 & 4345 & 63 \\
\hline $\mathrm{H}(65)$ & 2055 & 8192 & 4504 & 36 \\
\hline $\mathrm{H}(67 \mathrm{~A})$ & 1978 & 6663 & 5853 & 57 \\
\hline H(67B) & 1425 & 6433 & 5734 & 57 \\
\hline H(69) & 2659 & 7735 & 5935 & 47 \\
\hline $\mathrm{H}(70)$ & 3072 & 8470 & 5578 & 66 \\
\hline $\mathrm{H}(71)$ & 2738 & 8957 & 5504 & 56 \\
\hline $\mathrm{H}(72)$ & 1900 & 8622 & 5742 & 63 \\
\hline H(73) & 1478 & 7855 & 6054 & 56 \\
\hline $\mathrm{H}(75)$ & 2057 & 7651 & 7179 & 92 \\
\hline $\mathrm{H}(76)$ & 2253 & 7536 & 7999 & 86 \\
\hline $\mathrm{H}(77)$ & 2339 & 6832 & 8157 & 83 \\
\hline H(78) & 2135 & 6244 & 7502 & 97 \\
\hline H(79) & 1920 & 6398 & 6685 & 97 \\
\hline $\mathrm{H}(80 \mathrm{~A})$ & -178 & 6924 & 3469 & 41 \\
\hline $\mathrm{H}(80 \mathrm{~B})$ & -181 & 6588 & 3921 & 41 \\
\hline H(82) & 803 & 6220 & 2935 & 47 \\
\hline H(83) & 1526 & 6652 & 2477 & 62 \\
\hline $\mathrm{H}(84)$ & 1700 & 7354 & 2073 & 66 \\
\hline $\mathrm{H}(85)$ & 1201 & 7650 & 2036 & 78 \\
\hline H(86) & 421 & 7191 & 2449 & 68 \\
\hline H(88) & -222 & 6098 & 2034 & 59 \\
\hline H(89) & -884 & 5900 & 1477 & 114 \\
\hline $\mathrm{H}(90)$ & -1566 & 5865 & 1828 & 79 \\
\hline H(91) & -1574 & 6105 & 2640 & 74 \\
\hline $\mathrm{H}(92)$ & -953 & 6300 & 3217 & 68 \\
\hline $\mathrm{H}(96)$ & -928 & 4522 & 6495 & 78 \\
\hline H(98) & -970 & 3754 & 6453 & 109 \\
\hline H(99) & -634 & 3281 & 6145 & 85 \\
\hline $\mathrm{H}(100)$ & 115 & 3654 & 5705 & 77 \\
\hline $\mathrm{H}(101)$ & 503 & 4459 & 5649 & 76 \\
\hline $\mathrm{H}(106)$ & 2071 & 5840 & 5118 & 69 \\
\hline H(108) & 2331 & 5745 & 5992 & 85 \\
\hline H(109) & 2169 & 5481 & 6850 & 149 \\
\hline $\mathrm{H}(110)$ & 1349 & 5145 & 7197 & 129 \\
\hline $\mathrm{H}(111)$ & 763 & 5103 & 6618 & 84 \\
\hline $\mathrm{H}(11 \mathrm{~A})$ & -939 & 5267 & 6475 & 65 \\
\hline $\mathrm{H}(11 \mathrm{~B})$ & -631 & 5651 & 6044 & 65 \\
\hline $\mathrm{H}(115)$ & -553 & 5088 & 7486 & 85 \\
\hline $\mathrm{H}(116)$ & -120 & 4766 & 7887 & 86 \\
\hline $\mathrm{H}(117)$ & 718 & 5255 & 7907 & 107 \\
\hline $\mathrm{H}(118)$ & 1052 & 5988 & 7584 & 112 \\
\hline H(119) & 705 & 6318 & 7123 & 67 \\
\hline
\end{tabular}




\begin{tabular}{lrrrr}
$\mathrm{H}(121)$ & -431 & 6067 & 7929 & 80 \\
$\mathrm{H}(122)$ & -938 & 6209 & 8409 & 131 \\
$\mathrm{H}(123)$ & -1585 & 6199 & 8031 & 98 \\
$\mathrm{H}(124)$ & -1773 & 5957 & 7139 & 114 \\
$\mathrm{H}(125)$ & -1207 & 5854 & 6661 & 84 \\
$\mathrm{H}(12 \mathrm{~A})$ & 1848 & 6033 & 4304 & 54 \\
$\mathrm{H}(12 \mathrm{~B})$ & 1466 & 6199 & 4369 & 54 \\
$\mathrm{H}(128)$ & 269 & 4680 & 3826 & 57 \\
$\mathrm{H}(129)$ & -29 & 3884 & 4116 & 83 \\
$\mathrm{H}(130)$ & 526 & 3712 & 4502 & 70 \\
$\mathrm{H}(131)$ & 1305 & 4234 & 4565 & 57 \\
$\mathrm{H}(132)$ & 1583 & 4999 & 4294 & 51 \\
$\mathrm{H}(134)$ & 1327 & 5005 & 3039 & 58 \\
$\mathrm{H}(135)$ & 1789 & 5192 & 2285 & 81 \\
$\mathrm{H}(136)$ & 2428 & 6008 & 2138 & 75 \\
$\mathrm{H}(137)$ & 2520 & 6636 & 2686 & 100 \\
$\mathrm{H}(138)$ & 1979 & 6398 & 3435 & 71 \\
\hline
\end{tabular}

